\title{
Propriedades de Lie de elementos simétricos sob involuções orientadas em álgebras de grupo
}

\author{
John Hermes Castillo Gómez
}

TESE APRESENTADA

AO

Instituto De Matemática e Estatística

DA

Universidade DE SÃo Paulo

PARA

OBTENÇÃO DO TÍTULO

$\mathrm{DE}$

Doutor EM CIÊNCIAS

Programa: Matemática

Orientador: Prof. Dr. Francisco César Polcino Milies

Durante o desenvolvimento deste trabalho o autor recebeu auxílio financeiro da CAPES e do CNPq .

São Paulo, janeiro de 2013 


\title{
Propriedades de Lie de elementos simétricos sob involuções orientadas em álgebras de grupo
}

\author{
Esta tese contém as correções e alterações \\ sugeridas pela Comissão Julgadora durante a defesa \\ realizada por John Hermes Castillo Gómez em 29/11/2012. \\ O original encontra-se disponível no Instituto de \\ Matemática e Estatística da Universidade de São Paulo.
}

Comissão Julgadora:

- Prof. Dr. Francisco César Polcino Milies (orientador) - IME-USP

- Prof. Dr. Jairo Zacarias Gonçalves - IME-USP

- Prof ${ }^{\mathrm{a}}$ Dr$^{\mathrm{a}}$ Marinês Guerreiro - UFV

- Prof $^{\mathrm{a}}$ Dr$^{\mathrm{a}}$ Paula Murgel Veloso - UFF

- Prof. Dr. Antonio Paques - UFRGS 


\section{Agradecimentos}

A Dios por permitirme llegar tan lejos, donde nunca imagine.

Ao Professor César por ter aceitado me orientar, sem nem sequer me conhecer, por ter me proposto o problema de pesquisa, por suas indicações, por sua ajuda nas questões acadêmicas e especialmente nas burocráticas e principalmente por ser um amigo. Aos membros da Comissão Julgadora pelas observações e sugestões feitas que ajudaram a melhorar a apresentação desta tese.

À Coordenação de Aperfeiçoamento de Pessoal de Nivel Superior (CAPES) e ao Conselho Nacional de Desenvolvimento Científico e Tecnológico (CNPq) proc. 141857/2011-0: pelo apoio financeiro durante a elaboração deste trabalho.

À Catalina minha esposa, minha companheira e minha amiga: por sua energia constante, seu amor e sua dedicação para com nossa familia. Ao meu filho Simon, por me aceitar como sou, sua companhia durante estes anos no Brasil foram fundamentais: obrigado filho por me ensinar tantas coisas. À Francis por me escutar e por não responder, pois isso demonstraria finalmente minha loucura. Aos três Catalina, Simon e Francis por estar ao meu lado enquanto vivia no mundo da matemática: vocês são minha vida.

A mi familia en El Bordo, muchas gracias por siempre apoyarme en mis estudios. Gracias por entender mi ausencia y por la fuerza que me dan. Mamá y Papá perdon por no estar a su lado en los momentos dificiles que han vivido y a mis hermanas Beatriz y Lina gracias por cubrir mi ausencia. A mi familia en Medellín: Nubia, Walter, Ana, Paola, Farley, Alex, Pablo, Camila, Gustavo, familia Alvarez y familia Rúa muchas gracias por siempre estar ahí para ayudarnos.

Aos amigos no Ed. Star muito obrigado pelos momentos compartilhados, em especial à Pedro Bocaranda e Nohely Righi por sua amizade, comprensão e ajuda. Aos membros de plantão da CHURRASQUERIA do Star: Vitor, Claudia, Caio, William, Leandro, Priscila, Paulo Zanata (inho), Kika, Luciano, Cris, Angelica (ou Jaqueline kkk), Marcelo, Paulinha, Jaz e Raphael, Viviane e familia: valeu pelas cervejas, as carnes, o vinagrete, pelo truco (ainda que não aprendi a jogar-lo), etc.: em resumo valeu pela amizade.

Aos meus colegas e amigos de seminário no IME-USP: Alex (mi hermano del alma), Renata, Patricia, Fernanda, Anderson, Samir, Raúl e Gladys. Aos meus amigos no IME: Pri e Alvaro obrigado pela correção do português deste trabalho (os erros remanescentes são minha culpa) e pelos 5 anos (e pelos muitos que virão) de amizade sincera. A Luis Enrique, Javier e Aleja: por los momentos compartidos. A todos os funcionários do IME, em especial 
ao grupo de trabalho na CPG e na contabilidade: obrigado por facilitar com seu trabalho meu passo pelo instituto.

A la Universidad de Nariño por apoyarme durante mis estudios en Brasil. A todos los amigos en el Departamento de Matemáticas y Estadística en la Universidad de Nariño. En especial a quienes por uno u otro motivo me ayudaron: Claudia Patricia Gómez, Saulo Mosquera, Fernando Soto, Eduardo Ibarguen, Wilson Mutis, Fernando Benavides e Edwin Pacheco. A nuestra amiga Ohhh Lucy Aguilera: fuiste nuestro punto de apoyo en Colombia, como siempre gracias por todo.

Al grupo de investigación Álgebra, Teoría de Números y Aplicaciones, ERM y sus miembros en UNICAUCA, UNIVALLE, UDEA y UDENAR. En especial a mis eternos maestros y quienes me llevaron a continuar mis estudios: Carlos Alberto Trujillo Solarte y Gilberto García-Pulgarín. A mi colega, hermano y amigo: Juan Miguel Velásquez-Soto: gracias por tu amistad.

Existem muitas outras pessoas que me ajudaram ao longo do caminho e que gostaria agradecer aqui, mas acho que faltaria espaço: a todos muito obrigado.

São Paulo, Brasil Janeiro de 2013 
Arithmetic is where numbers run across your mind looking for the answer. Arithmetic is like numbers spinning in your head faster and faster until you blow up with the answer. KABOOM!!! Then you sit back down and begin the next problem. Alexander Nathanson 


\section{Resumo}

\section{Propriedades de Lie de elementos simétricos sob involuções orientadas em álgebras de grupo}

Sejam $F$ um corpo de característica diferente de 2 e $G$ um grupo. A partir da involução clássica, que envia cada elemento em seu inverso, e uma orientação do grupo $G$ é possível definir uma involução clássica orientada na álgebra de grupo $F G$. O objetivo desta tese é estudar propriedades de Lie do conjunto dos elementos simétricos $(F G)^{+}$e, em alguns casos, do conjunto dos elementos anti-simétricos $(F G)^{-}$.

Primeiro, abordamos o caso quando $G$ não tem elementos de ordem 2. Aqui, mostramos que se $(F G)^{+}\left(\right.$ou $\left.(F G)^{-}\right)$é Lie nilpotente ou Lie $n$-Engel, então $F G$ também é Lie nilpotente ou Lie $m$-Engel, respectivamente.

Depois, consideramos o caso quando $G$ contém uma cópia do grupo quatérnio de ordem 8. Neste caso, caracterizamos completamente as álgebras de grupo tais que $(F G)^{+}$é fortemente Lie nilpotente, Lie nilpotente e Lie $n$-Engel. Como consequência, provamos que o conjunto das unidades simétricas deste tipo de grupos é nilpotente.

Estudamos também o caso em que quando $G$ não contém uma cópia do grupo quatérnio de ordem 8. Em particular, apresentamos um exemplo que mostra que os resultados obtidos em pesquisas anteriores, com a involução clássica, não devem ser esperados ao trabalhar com involuções clássicas orientadas. Não entanto, damos alguns casos especiais de grupos nos quais esses resultados são obtidos.

Finalmente, estudamos o índice de Lie nilpotência de $(F G)^{+}$. Estabelecemos uma condição necessária e suficiente, para que o índice de Lie nilpotência de $(F G)^{+}$e a classe de nilpotência das unidades simétricas de uma álgebra de grupo Lie nilpotente sejam o maior possível. Além disso, consideramos a situação em que o grupo $G$ contém uma cópia de $Q_{8}$.

Palavras-chave: involução, orientação, álgebras de grupo, elemento simétrico, elemento anti-simétrico, Lie nilpotente, Lie $n$-Engel, fortemente Lie nilpotente, índice de Lie nilpotência. 


\section{Abstract}

\section{Lie properties of symmetric elements under oriented involutions in group algebras}

Let $F$ be a field of characteristic different from 2 and $G$ a group. From the classical involution, which sends each element in its inverse and an orientation of $G$, it is possible to define an oriented classical involution on the group algebra $F G$. The goal of this thesis is to study Lie properties of the set of symmetric elements $(F G)^{+}$and, in some cases, of the set of skew-symmetric elements $(F G)^{-}$.

We first deal with the case when $G$ does not have elements of order 2 . In this situation, we show that if $(F G)^{+}$(or $\left.(F G)^{-}\right)$is Lie nilpotent or Lie $n$-Engel, then the whole group algebra $F G$ satisfies the same property.

Later we consider the case when $G$ contains a copy of the quaternion group of order 8 . In this instance, we give a complete description of the group algebras such that $(F G)^{+}$is strongly Lie nilpotent, Lie nilpotent and Lie $n$-Engel. As a consequence, we get that the set of symmetric units of this kind of groups is nilpotent.

Furthermore, we study the case when $G$ does not contain a copy of the quaternion group of order 8. Here, we present an example that shows that the previews results obtained in former works, with the classical involution, may not hold with an oriented classical involution. However, we give some kinds of groups for which those results are achieved.

Finally, we study the Lie nilpotency index of $(F G)^{+}$. It is given a necessary and sufficient condition to the Lie nilpotency index of $(F G)^{+}$and the nilpotency class of the symmetric units to be maximal, in a Lie nilpotent group algebra. In addition, we consider the situation when $G$ contains a copy of the quaternion group of order 8 .

Keywords: involution, orientation, group algebras, symmetric element, skew-symmetric element, Lie nilpotent, Lie $n$-Engel, strongly Lie nilpotent, Lie nilpotency index. 


\section{Sumário}

Lista de Símbolos $\quad$ xi

1 Introdução 1

2 Conceitos preliminares $\quad 7$

2.1 Resultados conhecidos sobre propriedades de Lie . . . . . . . . . . . . . . . 7

2.2 Conceitos básicos . . . . . . . . . . . . . . . . . . . . 10

3 Propriedades de Lie e involuções clássicas orientadas $\quad 17$

3.1 Preliminares . . . . . . . . . . . . . . . . . . . . . . 17

3.2 Grupos sem elementos de ordem 2 . . . . . . . . . . . . . . . 25

4 Grupos em geral $\quad 29$

4.1 Grupos que contêm uma cópia de $Q_{8} \ldots \ldots \ldots$. . . . . . . . . . . . . 29

4.2 Outros resultados . . . . . . . . . . . . . . . . . . . . 36

5 Índices de Lie Nilpotência $\quad 39$

5.1 Preliminares . . . . . . . . . . . . . . . . . . . . . . . . 39

5.2 Álgebras de grupo Lie nilpotentes . . . . . . . . . . . . . . . . . . . . . 45

5.3 Grupos que contêm uma cópia de $Q_{8} \ldots \ldots \ldots$. . . . . . . . . . . . . . . 49

6 Conclusões $\quad 63$

6.1 Considerações finais . . . . . . . . . . . . . . . . . . . . 63

6.2 Sugestões para pesquisas futuras . . . . . . . . . . . . . . . . . 64

$\begin{array}{ll}\text { Referências Bibliográficas } & 65\end{array}$

$\begin{array}{ll}\text { Índice Remissivo } & 68\end{array}$ 


\section{Lista de Símbolos}

$\mathbb{N} \quad$ Conjunto dos números naturais.

$\mathbb{Z} \quad$ Conjunto dos números inteiros.

$\mathbb{Z}^{+} \quad$ Conjunto dos números inteiros positivos.

$\operatorname{car}(F) \quad$ Característica do corpo $F$.

$F G \quad$ Álgebra de grupo de $G$ sobre $F$.

$\Delta(G) \quad$ Ideal de aumento de $F G$.

$(F G)^{+} \quad$ Conjunto de elementos simétricos de $F G$.

$(F G)^{-} \quad$ Conjunto de elementos anti-simétricos de $F G$.

$G^{\prime} \quad$ Subgrupo comutador do grupo $G$.

$C_{G}(g) \quad$ Centralizador do elemento $g \in G$.

$[G: N] \quad$ Índice do subgrupo $N$ em $G$.

$\phi(G) \quad$ O FC subgrupo de $G$.

$\operatorname{Syl}_{\mathrm{p}}(G) \quad p$-subgrupo de Sylow de $G$.

$\zeta=\zeta(G)$ Centro do grupo $G$.

$\sigma \quad$ Orientação do grupo $G$.

* Involução clássica orientada.

$Q_{8} \quad$ Grupo quatérnio de ordem 8.

$D_{k} \quad$ Grupo diedral de ordem $2 k$.

$D_{\infty} \quad$ Grupo diedral infinito.

$S^{(n)} \quad n$-ésimo termo da série de potências de Lie de $S$.

$\mathrm{t}(S) \quad$ Índice de Lie nilpotência de $S$.

$\mathrm{t}^{\mathrm{L}}(S) \quad$ Índice de Lie nilpotência superior de $S$.

$\mathrm{t}_{\text {nil }}(P) \quad$ Índice de nilpotência do ideal de aumento $\Delta(P)$.

$\gamma_{n}(G) \quad n$-ésimo elemento da série central inferior de $G$.

$\operatorname{cl}(H) \quad$ Classe de nilpotência de $G$.

$D_{n}(F G) \quad n$-ésimo subgrupo dimensional.

$\Phi(G) \quad$ Subgrupo de Frattini de $G$.

$S_{r} \quad$ Grupo simétrico de grau $r$.

$\operatorname{sgn}(\tau) \quad$ Sinal da permutação $\tau$.

$S t_{r} \quad$ Identidade polinomial de grau $r$. 


\section{Capítulo 1}

\section{Introdução}

Seja $R$ um anel. Uma aplicação $*: R \rightarrow R$ diz-se uma involução; se

(i) $(r+s)^{*}=r^{*}+s^{*}$,

(ii) $(r s)^{*}=s^{*} r^{*} \mathrm{e}$

(iii) $\left(r^{*}\right)^{*}=r$;

para todo par de elementos $r, s$ em $R$.

Um elemento $r \in R$ diz-se simétrico sob $*$ se $r^{*}=r$. Além disso, um elemento $r \in R$ diz-se anti-simétrico sob $*$ quando $r^{*}=-r$. Denotaremos por $R^{+}=\left\{r \in R: r^{*}=r\right\}$ o conjunto dos elementos simétricos de $R$ sob $*$ e por $R^{-}=\left\{r \in R: r^{*}=-r\right\}$ o conjunto dos elementos anti-simétricos. Uma pergunta de interesse geral, na Teoria de Anéis, é quais propriedades de $R^{+}$ou $R^{-}$determinam propriedades de $R$. Um resultado clássico de Amitsur [1] afirma que se $R^{+}$(ou $R^{-}$) satisfaz uma identidade polinomial, então $R$ satisfaz uma identidade polinomial. É necessário notar que as referidas identidades polinomiais podem não ser iguais, como veremos adiante.

Sejam $R$ um anel com unidade e $G$ um grupo. O anel de grupo $R G$ é o conjunto de todas as somas formais

$$
\sum_{g \in G} \alpha_{g} g
$$

com cada $\alpha_{g} \in R$ e $\alpha_{g}=0$ quase sempre, isto é, somente um número finito de coeficientes são diferentes de zero em cada uma destas somas. Dado um elemento $\alpha=\sum_{g \in G} \alpha_{g} g$, o suporte 
de $\alpha$ é o conjunto de elementos em $G$ que efetivamente aparecem na expressão de $\alpha$, assim, $\operatorname{supp}(\alpha)=\left\{g \in G: \alpha_{g} \neq 0\right\}$.

O conjunto $R G$ tem uma estrutura de anel com as operações de soma e multiplicação definidas como segue:

$$
\sum_{g \in G} \alpha_{g} g+\sum_{g \in G} \beta_{g} g=\sum_{g \in G}\left(\alpha_{g}+\beta_{g}\right) g
$$

e

$$
\left(\sum_{g \in G} \alpha_{g} g\right)\left(\sum_{g \in G} \beta_{g} g\right)=\sum_{g, h \in G} \alpha_{g} \beta_{h} g h .
$$

Os elementos de $g \in G$ e $r \in R$ podem ser identificados com os elementos do anel de grupo $1 g$ e $r 1$, respectivamente.

Também pode-se definir um produto de elementos em $R G$ por elementos $\lambda \in R$ por $\lambda\left(\sum_{g \in G} \alpha_{g} g\right)=\sum_{g \in G}\left(\lambda \alpha_{g}\right) g$. Em consequência, $R G$ é um $R$-módulo. Além disso, se $R$ é um anel comutativo, $R G$ é uma álgebra sobre $R$.

É bem conhecido que em $R G$ é possível definir uma involução natural, que denominaremos involução clássica, dada por:

$$
\left(\sum_{g \in G} \alpha_{g} g\right)^{*}=\sum_{g \in G} \alpha_{g} g^{-1}
$$

Ao nosso entender esta involução surge na literatura pela primeira vez no artigo [18] de G. Higman em 1940. Em [21], Jespers e Ruiz deram uma generalização desta involução como segue: seja * uma involução do grupo $G$, define-se a involução de grupo * em $R G$ como sendo

$$
\left(\sum_{g \in G} \alpha_{g} g\right)^{*}=\sum_{g \in G} \alpha_{g} g^{*} .
$$

Em um anel associativo $R$, o colchete de Lie de dois elementos $x, y \in R$ é definido por $[x, y]=x y-y x$. Esta definição pode ser estendida recursivamente para $n \geq 2$ elementos de $R$, na seguinte forma $\left[x_{1}, \ldots, x_{n}\right]=\left[\left[x_{1}, \ldots, x_{n-1}\right], x_{n}\right]$. 
Para $X, Y \subseteq R$, por $[X, Y]$ denotamos o subgrupo aditivo gerado por todos os colchetes de Lie $[x, y]$, com $x \in X, y \in Y$. Seja $S$ um subconjunto não vazio de $R$. A série de Lie central inferior de $S$ é definida indutivamente por $\gamma^{1}(S)=S$ e $\gamma^{n+1}(S)=\left[\gamma^{n}(S), S\right]$, para $n \geq 1$. Dizemos que o subconjunto $S$ é Lie nilpotente, se existe um número natural $n$ tal que $\gamma^{n}(S)=0$. Pode-se mostrar que $S$ é Lie nilpotente se, e somente se, existe um $n \geq 2$ tal que $\left[a_{1}, \ldots, a_{n}\right]=0$ para todo $a_{1}, \ldots, a_{n} \in S$. O menor de tais $n$ é denominado o índice de Lie nilpotência de $S$ e será denotado por $\mathrm{t}(S)$. Definimos a série de potências de Lie de $S$ como: $S^{(1)}=S$ e, para $n \geq 2$, denotamos por $S^{(n)}$ o ideal associativo de $R$ gerado pelos colchetes de Lie $[x, y]$, com $x \in S^{(n-1)}, y \in S$. O conjunto $S$ é dito fortemente Lie nilpotente se algum membro da sua série de potências de Lie é zero, isto é, se existe $n$ tal que $S^{(n)}=0$. O menor de tais $n$ é denominado o índice de Lie nilpotência superior de $S$ e denotado por $\mathrm{t}^{\mathrm{L}}(S)$. Evidentemente, se $S$ é fortemente Lie nilpotente, então $S$ é Lie nilpotente e $\mathrm{t}(S) \leq \mathrm{t}^{\mathrm{L}}(S)$, mas a igualdade nem sempre é satisfeita.

Dado um inteiro positivo $n$, dizemos que $S$ é Lie $n$-Engel se

$$
[a, \underbrace{b, \ldots, b}_{n \text { vezes }}]=0
$$

para todo $a, b \in S$. Obviamente, o fato de um conjunto $S$ ser Lie nilpotente implica que também é Lie $n$-Engel, para algum $n$.

Sejam $G$ um grupo e $g_{1}, \ldots, g_{n} \in G$. Com o símbolo $\left(g_{1}, \ldots, g_{n}\right)$ denotamos o comutador dos elementos $g_{1}, \ldots, g_{n}$, que definimos, indutivamente, por $\left(g_{1}, \ldots, g_{n}\right)=\left(\left(g_{1}, \ldots, g_{n-1}\right), g_{n}\right)$, com $\left(g_{1}, g_{2}\right)=g_{1}^{-1} g_{2}^{-1} g_{1} g_{2}$. Como é usual, para subconjuntos $X, Y$ de $G$, pelo comutador $(X, Y)$ denotamos o subgrupo gerado pelos comutadores $(x, y)$, com $x \in X, y \in Y$. Isto nos permite definir a série central inferior de um subconjunto $H$ de $G: \gamma_{1}(H)=H$ e $\gamma_{n+1}(H)=\left(\gamma_{n}(H), H\right)$, para $n \geq 1$. Dizemos que $H$ é nilpotente, se $\gamma_{n}(H)=1$ para algum $n$. É fácil provar que as seguintes afirmações são equivalentes [24, Lema 4.1.2]:

(i) $H$ é nilpotente;

(ii) $H$ satisfaz a identidade de grupo $\left(g_{1}, g_{2}, \ldots, g_{n}\right)=1$, para algum $n \geq 2$

(iii) $\langle H\rangle$ é um grupo nilpotente. 
Para um subconjunto nilpotente $H \subseteq G$, o número $\operatorname{cl}(H)=\min \left\{n \in \mathbb{N}_{0}: \gamma_{n+1}(H)=1\right\}$ é denominado a classe de nilpotência de $H$.

Novamente seja $S$ um subconjunto de um anel associativo $R$. Denotamos por

$$
\begin{aligned}
& \mathcal{U}(S)=\{u \in S:(\exists v \in S) \quad u v=v u=1\} \mathrm{e} \\
& \mathcal{U}^{+}(S)=\mathcal{U}(S) \cap S^{+}
\end{aligned}
$$

o conjunto de unidades e o conjunto de unidades simétricas de $S$, respectivamente. Suponha que $\mathcal{U}(S)$ é não vazio. Pela igualdade, $(x, y)=1+x^{-1} y^{-1}[x, y]$, onde $x, y \in \mathcal{U}(S)$, obtemos a inclusão $\gamma_{n}(\mathcal{U}(S)) \subseteq 1+S^{(n)}$, para $n \geq 2$, e daí o conjunto de unidades de um subconjunto fortemente Lie nilpotente é um conjunto nilpotente e $\operatorname{cl}(\mathcal{U}(S))+1 \leq \mathrm{t}^{\mathrm{L}}(S)$.

Em 1973, Passi, Passman e Sehgal [27] mostraram que uma álgebra de grupo FG é Lie nilpotente se, e somente se, $G$ é nilpotente e $G^{\prime}$ é um $p$-grupo finito, onde $p$ é a característica de F. Posteriormente Sehgal [32] mostrou que os conceitos de Lie nilpotência e Lie nilpotência forte são equivalentes em álgebras de grupo e caracterizou as álgebras de grupo que são Lie $n$-Engel, para algum $n \in \mathbb{N}$.

Em 1993, Giambruno e Sehgal começaram o estudo da Lie nilpotência de elementos simétricos e anti-simétricos sob a involução clássica. Em [15], eles provaram que dado um grupo $G$ sem elementos de ordem 2 e um corpo $F$ de $\operatorname{car}(F) \neq 2$, se $(F G)^{+}$ou $(F G)^{-}$ é Lie nilpotente, então $F G$ é Lie nilpotente. Este trabalho foi concluído por Lee em [22], para grupos em geral. Mais especificamente, ele provou que a Lie nilpotência dos elementos simétricos sob a involução clássica é equivalente à Lie nilpotência de $F G$ quando o grupo $G$ não contém uma cópia de $Q_{8}=\left\langle x, y: x^{4}=1, x^{2}=y^{2}, x^{y}=x^{-1}\right\rangle$, o grupo quatérnio de ordem 8 e também caracterizou as álgebras de grupo tais que os elementos simétricos são Lie nilpotentes, quando $G$ contém uma cópia de $Q_{8}$. Note que neste caso, $(F G)^{+}$e $F G$ não satisfazem a mesma identidade polinomial. A propriedade Lie $n$-Engel foi estudada por Lee em [23], com resultados similares.

Em [13], Giambruno, Sehgal e Polcino Milies estudaram estas propriedades no conjunto dos elementos simétricos sob uma involução de grupo, quando $G$ é um grupo sem elementos de ordem 2. Este trabalho foi concluído, para grupos em geral, por Lee, Sehgal e Spinelli em 
$[25]$.

É claro que um caso particular de Lie nilpotência, e obviamente da propriedade Lie n-Engel, é a comutatividade. A comutatividade dos elementos simétricos sob a involução clássica e no caso das involuções de grupo foi estudada por Broche Cristo em [7] e por Jespers e Ruiz Marin em [21], respectivamente.

Existem outras involuções em anéis de grupos que generalizam as involuções de grupo. Sejam $R$ um anel de característica diferente de 2 e $G$ um grupo. Um homomorfismo $\sigma: G \rightarrow\{ \pm 1\}$ é chamado uma orientação do grupo $G$. Seja * uma involução do grupo $G$. Em [10], Broche Cristo e Polcino Milies consideraram uma involução de grupo orientada, como segue:

$$
\left(\sum_{g \in G} \alpha_{g} g\right)^{*}=\sum_{g \in G} \alpha_{g} \sigma(g) g^{*} .
$$

Se * é a involução que envia cada elemento de $G$ em seu inverso, nos referiremos a este tipo de involução como uma involução clássica orientada de $F G$. Tudo indica que este tipo de involuções foi introduzida em 1970 por Novikov [26]. Além disso, se $\sigma$ é trivial, esta involução coincide com a involução clássica de $R G$.

Em [10], Broche Cristo e Polcino Milies, caracterizaram as álgebras de grupo $F G$ tais que $(F G)^{+}$sob uma involução de grupo orientada é comutativo. Também existem trabalhos nos quais se estudam propriedades de Lie e a comutatividade do conjunto de elementos anti-simétricos nas diferentes involuções que foram definidas acima, [9, 12, 15, 16] e [8].

Outro aspecto que vem sendo considerado é o cálculo dos números $\operatorname{cl}(S), \mathrm{t}(S)$ e $\mathrm{t}^{\mathrm{L}}(S)$, para subconjuntos $S$ de uma álgebra de grupo FG. Assim, por exemplo, no artigo [33] de Sharma e Bist prova-se que se $F G$ é Lie nilpotente, então $\mathrm{t}^{\mathrm{L}}(F G) \leq\left|G^{\prime}\right|+1$ e, Bovdi e Spinelli, [6] mostraram os casos em que a igualdade é alcançada. Recentemente, Balogh e Juhász [5], [4] estudaram o índice de Lie nilpotência de $(F G)^{+}$e a classe de nilpotência de $\mathcal{U}^{+}(F G)$ sob a involução clássica.

O objetivo desta tese é estudar as propriedades de Lie, definidas anteriormente, dos elementos simétricos sob uma involução clássica orientada. Em alguns casos, também são obtidos resultados para o conjunto dos elementos anti-simétricos. 
No Capítulo 2, expomos uma revisão de conceitos preliminares de anéis de grupo que serão usados neste texto. Em especial, lembramos alguns dos resultados conhecidos na literatura sobre propriedades de Lie em álgebras de grupo.

No Capítulo 3, começamos o estudo de propriedades de Lie e involuções clássicas orientadas. Como principal resultado da Seção 3.1, ver Proposição 3.1.6, provamos que se $G$ é um grupo tal que $\zeta(G)^{2}=\left\{z^{2}: z \in \zeta(G)\right\}$ é infinito, os índices de Lie nilpotência de $(F G)^{+}$ e FG são iguais. Na Seção 3.2 consideramos grupos que não têm elementos de ordem 2. Em particular nos teoremas 3.2.6 e 3.2.5, provamos que se $(F G)^{+}$ou $(F G)^{-}$é Lie nilpotente (Lie $n$-Engel), então $F G$ também é Lie nilpotente (Lie $m$-Engel, para algum $m$ ). É bom destacar aqui que os resultados obtidos neste capítulo estendem os resultados obtidos em [15] e que algumas das nossas demonstrações estão baseadas naquelas ali apresentadas.

No Capítulo 4, abordamos o problema para grupos em geral. Na Seção 4.1 damos a resposta completa quando o grupo $G$ contém uma cópia do grupo quatérnio de ordem 8, ver teoremas 4.1.5, 4.1.6 e 4.1.8. Em especial, o Teorema 4.1 .8 implica que os conceitos de Lie nilpotência e Lie nilpotência forte são equivalentes no conjunto dos elementos simétricos e, que o conjunto de unidades simétricas nesta classe de grupos es nilpotente. Na Seção 4.2, estudamos o problema quando $G$ não contém uma cópia do grupo quatérnio de ordem 8 e damos algumas respostas parciais para resolver o problema neste caso, ver teoremas 4.2.2, 4.2.3 e Proposição 4.2.4. No começo dessa seção, damos um exemplo de uma álgebra de grupo $F G$ tal que $G$ não contém uma cópia de $Q_{8}$ e $(F G)^{+}$é Lie $n$-Engel, para $n \geq 2$, mas $F G$ não é Lie $k$-Engel, para qualquer $k$. A importância deste exemplo é que os resultados obtidos por Lee nos artigos [22, 23] podem não valer quando trabalhamos com involuções clássicas orientadas. Os resultados expostos nesse capítulo estendem resultados de Lee [22, 23] e [24]. Alguns dos resultados dos Capítulos 3 e 4 serão publicados no artigo [11].

No Capítulo 5, exploramos o índice de Lie nilpotência de $(F G)^{+}$e a classe de nilpotência de $\mathcal{U}^{+}(F G)$. Em particular, no Teorema 5.2.2 mostramos que o índice de Lie nilpotência de $(F G)^{+}$e a classe de nilpotência de $\mathcal{U}^{+}(F G)$ em uma álgebra Lie nilpotente são os maiores possíveis se, e somente, se $G^{\prime}$ é cíclico. Na Seção 5.3, exploramos o mesmo problema quando $G$ contém uma cópia do grupo quatérnio de ordem 8, ver Teorema 5.3.7. Os resultados expostos neste capítulo estendem resultados obtidos por Balogh e Juhász [5]. 


\section{Capítulo 2}

\section{Conceitos preliminares}

Na primeira seção deste capítulo lembramos os resultados prévios já conhecidos na literatura que serão usados durante este trabalho. Na segunda seção damos alguns resultados técnicos que usaremos nos próximos capítulos.

\subsection{Resultados conhecidos sobre propriedades de Lie}

Sejam $F$ um corpo, $\mathcal{A}$ uma $F$-álgebra e $F\left\{x_{1}, x_{2}, \ldots\right\}$ a álgebra livre sobre variáveis não comutantes $x_{i}$. Dizemos que $\mathcal{A}$ satisfaz uma identidade polinomial ou que é uma PI-álgebra se existe um polinômio não nulo $f\left(x_{1}, \ldots, x_{n}\right) \in F\left\{x_{1}, x_{2}, \ldots\right\}$ tal que $f\left(a_{1}, \ldots, a_{n}\right)=0$, para todos $a_{1}, \ldots, a_{n} \in \mathcal{A}$. Similarmente, se $\Lambda$ é um subconjunto de $\mathcal{A}$, então $\Lambda$ satisfaz uma identidade polinomial $f$, se $f\left(\lambda_{1}, \ldots, \lambda_{n}\right)=0$, para todos $\lambda_{1}, \ldots, \lambda_{n} \in \Lambda$.

Dado um grupo $G$, por $\phi(G)=\left\{g \in G:\left[G: C_{G}(g)\right]<\infty\right\}$ denotamos o FC subgrupo de $G$, onde $C_{G}(g)=\{x \in G: x g=g x\}$ e $\left[G: C_{G}(g)\right]$ é o índice de $C_{G}(g)$ em $G$. Também por $G^{\prime}$ denotamos o subgrupo comutador de $G$. A caracterização de quando uma álgebra de grupo FG é uma PI-álgebra foi dada por Isaacs e Passman para característica 0 [19] e por Passman [28] para característica diferente de 0, veja [29, Corolário V.3.8 e Corolário V.3.10]. Lembramos que um grupo $G$ é denominado $p$-abeliano quando $G^{\prime}$ é um $p$-grupo finito. Quando $p=0$, 0-abeliano significará abeliano.

Teorema 2.1.1 (Corolário V.3.8 e Corolário V.3.10, [29]). Seja F um corpo de característica p. Então FG é uma PI-álgebra se, e somente se, $G$ tem um subgrupo p-abeliano de indice 
finito.

As condições sob as quais $F G$ é Lie nilpotente ou Lie $n$-Engel foram determinadas em artigos clássicos. Em 1973, Passi, Passman e Sehgal [27] caracterizaram quando FG é Lie nilpotente e Sehgal [32], mostrou que os conceitos de Lie nilpotência e Lie nilpotência forte são equivalentes em álgebras de grupo. Além disso, ele encontrou as condições necessárias para que $F G$ seja Lie $n$-Engel. A seguir enunciamo estes resultados.

Teorema 2.1.2 (Teorema V.4.4, [32]). Sejam F um corpo de característica $p \geq 0$ e Gum grupo. As seguintes afirmações são equivalentes

(i) FG é Lie nilpotente;

(ii) FG é fortemente Lie nilpotente;

(iii) $G$ é nilpotente e p-abeliano.

Teorema 2.1.3 (Teorema V.6.1, [32]). Se car $(F)=0$, então FG é Lie n-Engel, para algum $n \in \mathbb{N}$, se, e somente se, $G$ é abeliano. Se $\operatorname{car}(F)=p>0$, então $F G$ é Lie $n$-Engel, para algum $n \in \mathbb{N}$, se e somente se, $G$ é nilpotente e $G$ tem um subgrupo normal p-abeliano de indice uma potência de p.

Sejam $G$ um grupo e $F$ um corpo de característica diferente de 2. Um homomorfismo $\sigma: G \rightarrow\{ \pm 1\}$ é chamado uma orientação do grupo $G$. Seja $*$ uma involução do grupo $G$. Define-se a involução clássica orientada, por

$$
\left(\sum_{g \in G} \alpha_{g} g\right)^{*}=\sum_{g \in G} \alpha_{g} \sigma(g) g^{-1} .
$$

Note que se $\sigma$ é trivial esta involução coincide com a involução clássica de $F G$.

Com $N$ denotamos o núcleo de $\sigma$. Na álgebra de grupo $F N$, a involução * coincide com a involução clássica. É fácil ver que, como um $F$-módulo, $(F G)^{+}$é gerado pelo conjunto

$$
\mathcal{S}=\left\{g \in N: g^{2}=1\right\} \cup\left\{g+g^{-1}: g \in N, g^{2} \neq 1\right\} \cup\left\{g-g^{-1}: g \in G \backslash N, g^{2} \neq 1\right\},
$$


e $(F G)^{-}$é gerado por

$$
\mathcal{L}=\left\{g \in G \backslash N: g^{2}=1\right\} \cup\left\{g+g^{-1}: g \in G \backslash N, g^{2} \neq 1\right\} \cup\left\{g-g^{-1}: g \in N, g^{2} \neq 1\right\}
$$

Lee, [22, 23] e [24], caracterizou quando o conjunto dos elementos simétricos sob a involução clássica é Lie nilpotente ou Lie $n$-Engel, para algum $n \in \mathbb{N}$ dado. Os resultados de Lee são:

Teorema 2.1.4 (Teorema 1, [22]). Sejam F um corpo com característica diferente de 2 e G um grupo tal que $(F G)^{+}$é Lie nilpotente. Se $Q_{8} \nsubseteq \mathbb{G}$, então FG é Lie nilpotente.

Teorema 2.1.5 (Teorema 2, [22]). Sejam F um corpo com característica diferente de 2 e G um grupo tal que $Q_{8} \subseteq G$. Então, $(F G)^{+}$é Lie nilpotente (fortemente Lie nilpotente) se, e somente uma das seguintes afirmações é válida

(i) $\operatorname{car}(F)=0$ e $G \simeq Q_{8} \times E$, onde $E^{2}=1$ ou

(ii) $\operatorname{car}(F)=p>2$ e $G \simeq Q_{8} \times E \times P$, onde $E^{2}=1$ e $P$ é um p-grupo finito.

Teorema 2.1.6 (Teorema 1, [23]). Sejam F um corpo com característica diferente 2 e $G$ um grupo tal que $(F G)^{+}$é Lie n-Engel, para algum $n \in \mathbb{N}$. Se $Q_{8} \nsubseteq$ G, então FG é Lie $m$-Engel, para algum $m \in \mathbb{N}$.

Teorema 2.1.7 (Teorema 2, [23]). Sejam F um corpo de característica diferente de 2 e $G$ um grupo tal que $Q_{8} \subseteq G$. Então, $(F G)^{+}$é Lie $n$-Engel, para algum $n \in \mathbb{N}$, se, e somente se, vale uma das seguintes condições

(i) $\operatorname{car}(F)=0$ e $N \simeq Q_{8} \times E$, onde $E^{2}=1$ ou

(ii) $\operatorname{car}(F)=p>2$ e $N \simeq Q_{8} \times E \times P$, onde $E^{2}=1$ e $P$ é um p-grupo nilpotente de expoente limitado que contém um subgrupo normal p-abeliano $A$ de indice finito.

Seja $r \in \mathbb{Z}^{+}$. O grupo de todas as permutações do conjunto $\{1, \ldots, r\}$ será denotado por $S_{r}$ e denominado como grupo simétrico de grau $r$. Para qualquer $r \in \mathbb{Z}^{+}$, a identidade polinomial standard de grau $r$ é

$$
S t_{r}\left(x_{1}, \ldots, x_{r}\right)=\sum_{\tau \in S_{r}} \operatorname{sgn}(\tau) x_{\tau(1)} x_{\tau(2)} \cdots x_{\tau(r)},
$$


onde $\operatorname{sgn}(\tau)$ denota o sinal da permutação $\tau$.

Giambruno et al. [13] provaram o seguinte resultado, o qual permite estabelecer uma conexão entre as propriedades de Lie dos elementos simétricos em um anel semiprimo com a sua comutatividade.

Teorema 2.1.8 (Lema 2.4, [13]). Seja $R$ um anel semiprimo com involução tal que $2 R=R$. Se $R^{+}$(respectivamente $R^{-}$) é Lie $n$-Engel, para algum $n \in \mathbb{N}$, então $R^{+}$(respectivamente $R^{-}$) é comutativo e $R$ satisfaz a identidade polinomial standard $\mathrm{St}_{4}$ de grau 4 .

A comutatividade é um caso particular das propriedades de Lie nilpotência e Lie $n$-Engel. A classificação das álgebras de grupo tais que os elementos simétricos comutam com respeito a uma involução clássica orientada foi dada em [10] por Broche Cristo e Polcino Milies.

Teorema 2.1.9. Seja $R$ um anel comutativo com unidade e seja $G$ um grupo não abeliano com um homomorfismo de orientação não trivial $\sigma$. Então, $(R G)^{+}$é um anel comutativo se, e somente se, temos uma das seguintes condições:

(i) $N=\operatorname{ker}(\sigma)$ é um grupo abeliano $e(G \backslash N)^{2}=1$;

(ii) $N=\operatorname{ker}(\sigma) \simeq\left\langle x, y: x^{4}=1, y^{2}=x^{2}, y^{-1} x y=x^{-1}\right\rangle \times E$ e $G \simeq\left\langle x, y, g: x^{4}=1, y^{2}=\right.$ $\left.x^{2}=g^{2}, x^{y}=x^{-1}, x^{g}=x, y^{g}=y\right\rangle \times E$, onde E é um 2-grupo abeliano elementar;

(iii) $\operatorname{car}(R)=4$ e G é um 2-grupo Hamiltoniano.

\subsection{Conceitos básicos}

Sejam $G$ um grupo e $F$ um corpo de característica diferente de 2. Seja $H$ um subgrupo de $G$. Claramente $F H$ é uma subálgebra de $F G$. Existe uma projeção natural $\pi_{H}: F G \rightarrow F H$ dada por

$$
\pi_{H}\left(\sum_{x \in G} a_{x} x\right)=\sum_{x \in H} a_{x} x .
$$

Em outras palavras, se $\alpha \in F G$, então $\alpha=\pi_{H}(\alpha)+\alpha^{\prime}$ onde $\operatorname{supp}\left(\alpha^{\prime}\right)$ é disjunto de $H$ e $\operatorname{supp}\left(\alpha-\alpha^{\prime}\right) \subseteq H$. Apresentamos algumas propriedades básicas de $\pi_{H}$.

Lema 2.2.1 (Lema I.1.2, [29]). Seja $H$ um subgrupo de $G$ e sejam $\alpha, \beta \in F G, \gamma \in F H$. Então: 
1. $\pi_{H}(a \alpha+b \beta)=a \pi_{H}(\alpha)+b \pi_{H}(\beta)$, para todos $a, b \in F$.

2. $\pi_{H}(\gamma \alpha)=\gamma \pi_{H}(\alpha), \pi_{H}(\alpha \gamma)=\pi_{H}(\alpha) \gamma$.

3. Se $H$ é um subgrupo normal de $G$ e se $x \in G$, então

$$
\pi_{H}\left(x^{-1} \alpha x\right)=x^{-1} \pi_{H}(\alpha) x .
$$

Usando o lema anterior podemos provar o seguinte resultado.

Lema 2.2.2. Sejam $H$ um subgrupo de $G$ e $\alpha \in F H$. Então $\alpha$ é um divisor de zero à direita (ou à esquerda) em FH se, e somente se, é um divisor de zero à direita (ou à esquerda) em $F G$.

Demonstração. Obviamente, se $\alpha$ é divisor de zero à direita em $F H$, dado que $F H \subseteq F G$, $\alpha$ é um divisor de zero à direita em $F G$.

Suponha $\alpha \beta=0$, para algum $0 \neq \beta \in F G$. Então podemos escolher $x \in G$ tal que $1 \in \operatorname{supp}(\beta x)$. Como $\alpha(\beta x)=0$ temos

$$
\alpha \pi_{H}(\beta x)=\pi_{H}(\alpha \beta x)=\pi_{H}(0)=0
$$

e $\pi_{H}(\beta x) \neq 0$, pois $1 \in \operatorname{supp}(\beta x)$. Isto completa a prova.

Lema 2.2.3. Se $G=\langle x\rangle$ é um grupo cíclico infinito, então $F G$ não tem divisores de zero.

Demonstração. Sejam $\alpha$ e $\beta$ elementos não nulos em $F G$. Logo, para $n, m \in \mathbb{N}$ apropriados $\alpha=\sum_{i \leq n} a_{i} x^{i}$ e $\beta=\sum_{j \leq m} b_{j} x^{j}$, com $a_{n}, b_{m} \neq 0$. Desta forma, o coeficiente de $x^{n+m}$ em $\alpha \beta$ não é zero e, portanto, $\alpha \beta \neq 0$.

Lema 2.2.4. Sejam $R$ um anel associativo e $S$ um subconjunto de $R$ tal que $\mathcal{U}(S)$ é não vazio. Então, para todo inteiro positivo $n, \gamma_{n}(\mathcal{U}(S)) \subseteq 1+S^{(n)}$. Em particular, se $S$ é fortemente Lie nilpotente, então $\mathcal{U}(S)$ é nilpotente e $\operatorname{cl}(\mathcal{U}(S))+1 \leq \mathrm{t}^{\mathrm{L}}(S)$.

Demonstração. A prova é por indução sobre $n$. O caso $n=1$ é trivial. Tome $u \in \gamma_{n}(\mathcal{U}(S))$, $v \in \mathcal{U}(S)$. Então

$$
(u, v)-1=u^{-1} v^{-1}[u, v]=u^{-1} v^{-1}[u-1, v] .
$$


Pela hipótese de indução, $u-1 \in S^{(n)}$, $\operatorname{logo}(u, v) \in 1+S^{(n+1)}$. Como $S^{(n+1)}$ é um ideal, podemos ver que produtos e inversos de unidades em $1+S^{(n+1)}$ também estão em $1+S^{(n+1)}$.

Usaremos o seguinte lema em diferentes situações.

Lema 2.2.5. Seja $R$ um anel de característica p. Então para qualquer $a, b \in R$ e qualquer inteiro positivo $n$, temos

$$
[a, \underbrace{b, \ldots, b}_{p^{n} \text { vezes }}]=\left[a, b^{p^{n}}\right] .
$$

Demonstração. Seja $b \in R$ fixo. Seja $r_{b}: R \rightarrow R$ definido por $r_{b}(c)=c b$, para todo $c \in R$. Similarmente podemos definir o operador $l_{b}: R \rightarrow R$ por $l_{b}(c)=b c$, para todo $c \in R$. Então, $[c, b]=\left(r_{b}-l_{b}\right)(c)$ para todo $c \in R$. Assim:

$$
[a, \underbrace{b, \ldots, b}_{p^{n} \text { vezes }}]=[[\cdots[[a, b], b], \cdots], b]=\left(r_{b}-l_{b}\right)^{p^{n}}(a) .
$$

Como $r_{b}$ e $l_{b}$ comutam como operadores e $\operatorname{car}(R)=p$, segue que $[a, \underbrace{b, \ldots, b}_{p^{n} \text { vezes }}]=\left(r_{b}^{p^{n}}-l_{b}^{p^{n}}\right)(a)=$ $a b^{p^{n}}-b^{p^{n}} a=\left[a, b^{p^{n}}\right]$

Definição 2.2.6. Sejam $R$ um anel e $G$ um grupo. O homomorfismo $\varepsilon: R G \rightarrow R$ dado por

$$
\varepsilon\left(\sum_{g \in G} a_{g} g\right)=\sum_{g \in G} a_{g}
$$

é denominado aplicação de aumento de $R G$ e seu núcleo, denotado por $\Delta(G)$, é o ideal de aumento de $R G$.

O seguinte resultado mostra como é está constituído o ideal de aumento de $R G$.

Proposição 2.2.7 (Proposição 3.2.10, [30]). O conjunto $\{g-1: g \in G, g \neq 1\}$ é uma base de $\Delta(G)$ sob $R$.

Para um subgrupo $H$ de $G$, denotamos por $\Delta(G, H)$ o ideal esquerdo de $R G$ gerado pelo conjunto $\{h-1: h \in H\}$. Isto é,

$$
\Delta(G, H)=\left\{\sum_{h \in H} \alpha_{h}(h-1): \alpha_{h} \in R G\right\}
$$


Agora damos uma interpretação para $\Delta(G, H)$ quando $H$ é um subgrupo normal de $G$. De fato, se $H \triangleleft G$, então o homomorfismo natural $G \rightarrow G / H$, que envia cada elemento de $G$ na sua classe lateral em $G / H$, induz um homomorfismo de anéis $\varepsilon_{H}: R H \rightarrow R(G / H)$ como segue:

$$
\varepsilon_{H}\left(\sum_{g \in G} \alpha_{g} g\right)=\sum_{g \in G} \alpha_{g} g H
$$

Com a notação anterior:

Proposição 2.2.8 (Proposição 3.3.4, [30]). Se $H$ é um subgrupo normal de $H$, então $\Delta(G, H)$ é o núcleo do homomorfismo $\varepsilon_{H}$.

Dado um grupo $G$, podemos definir, a partir de uma cadeia de ideais contidos no ideal de aumento $\Delta(G)$ na álgebra de grupo $F G$, uma cadeia de subgrupos do grupo base $G$ e vice-versa.

Definição 2.2.9. Considere a álgebra de grupo FG. Uma filtração (também conhecida como graduação) de $\Delta(G)$ é uma sequência de ideais de FG tal que

$$
\Delta(G)=E_{1} \supseteq E_{2} \supseteq \cdots \supseteq E_{n} \supseteq \cdots
$$

e $E_{i} E_{j} \subseteq E_{i+j}$ para todo $i, j \in \mathbb{N}$.

De muita importância é a filtração formada pelas potências do ideal de aumento, isto é,

$$
\Delta(G) \supseteq \Delta(G)^{2} \supseteq \cdots \supseteq \Delta(G)^{k} \supseteq \cdots
$$

Uma filtração de $\Delta(G)$ leva a definir uma sequência $\left\{G_{i}\right\}$ de subgrupos de $G$ como segue:

$$
G_{i}=\left\{x \in G: x-1 \in E_{i}\right\}
$$

A sequência $\left\{G_{i}\right\}$ satisfaz as seguintes propriedades.

Lema 2.2.10 (Lema III.3.1, [29].). Com a notação acima:

(i) $G=G_{1} \supseteq G_{2} \supseteq \cdots G_{n} \supseteq \cdots$ é uma cadeia decrescente de subgrupos normais de $G$. 
(ii) Para todos $i, j \in \mathbb{N},\left(G_{i}, G_{j}\right) \subseteq G_{i+j}$.

(iii) Se $\operatorname{car}(F)=p$ e $x \in G_{i}$, então $x^{p} \in G_{i p}$.

Definição 2.2.11. Seja $G=G_{1} \supseteq G_{2} \supseteq \cdots G_{n} \supseteq \cdots$ uma sequência de subgrupos normais de $G$. Então $\left\{G_{i}\right\}$ é denominada uma $N$-sequência se $\left(G_{i}, G_{j}\right) \subseteq G_{i+j}$ para todos $i, j \in \mathbb{N}$. Além disso, dado um primo p, dizemos que $\left\{G_{i}\right\}$ é uma $N_{p}$-sequência se é uma $N$-sequência e $x^{p} \in G_{i p}$, sempre que $x \in G_{i}$.

Desta forma, do Lema 2.2.10 qualquer filtração de $\Delta(G)$ define uma $N$-sequência em $G$ e quando $\operatorname{car}(F)=p$ define uma $N_{p}$-sequência. Em particular, a filtração (2.2) define a cadeia de subgrupos, conhecidos como subgrupos dimensionais de $G$, definida por

$$
D_{i}(F G)=\left\{x \in G: x-1 \in \Delta(G)^{i}\right\}
$$

Assim temos o seguinte lema.

Lema 2.2.12. Os subgrupos dimensionais $D_{n}(F G)$ formam uma cadeia decrescente de subgrupos normais de $G$ começando com $D_{1}(F G)=G$. Além disso, se car $(F)=p$, esta é uma $N_{p}$-sequência.

Para o restante desta seção $G$ será um p-grupo finito. Já vimos que dada uma filtração de $\Delta(G)$ obtemos uma $N_{p^{-}}$sequência. Ainda podemos fazer o caminho inverso, isto é, a partir de uma $N_{p}$-sequência podemos construir uma filtração de $\Delta(G)$.

Seja

$$
G=G_{1} \supseteq G_{2} \supseteq \cdots G_{d} \supseteq G_{d+1}=\langle 1\rangle
$$

uma $N_{p}$-sequência de $G$. Para cada $g \in G$, definimos a altura de $g, \nu(g)$, como o maior sub-índice $m$ para o qual $g \in G_{m}$. Assim $\nu(1)=\infty$, pois a cadeia é realmente uma sequência infinita que se repete depois de $i=d+1$ e, para $g \neq 1, \nu(g)=m$ se $g \in G_{m}$, mas $g \notin G_{m+1}$.

Agora, para um inteiro $i \geq 1$, seja $E_{i} \subseteq F G$ o $F$-espaço vetorial gerado por todos os produtos da forma

$$
\left(g_{1}-1\right)\left(g_{2}-1\right) \cdots\left(g_{k}-1\right)
$$


para algum $k, \operatorname{com} \nu\left(g_{1}\right)+\nu\left(g_{2}\right)+\cdots+\nu\left(g_{k}\right) \geq i$. Claramente, $E_{i}$ é um ideal de $F G$ porque é fechado sob a multiplicação por 1 e $g-1$. Além disso, temos

$$
\Delta(G)=E_{1} \supseteq E_{2} \supseteq \cdots \supseteq E_{i} \supseteq \cdots
$$

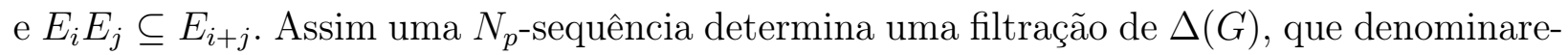
mos a filtração determinada por $\left\{G_{i}\right\}$.

Necessitamos de mais um conceito. Para poder definí-lo apresentamos o seguinte resultado.

Lema 2.2.13 (Lema III.3.3, [29]). Sejam $|G|=p^{n}$ e $G=H_{1}>H_{2}>\cdots>H_{n+1}=\langle 1\rangle$ uma série de subgrupos com $H_{i+1} \triangleleft H_{i}$ e $\left|H_{i} / H_{i+1}\right|=p$. Para cada $i \geq 1$, escolha $x_{i} \in H_{i} \backslash H_{i+1}$. Então os elementos

$$
\eta\left(a_{1}, a_{2}, \ldots, a_{n}\right)=\left(x_{1}-1\right)^{a_{1}}\left(x_{2}-1\right)^{a_{2}} \cdots\left(x_{n}-1\right)^{a_{n}}
$$

com $0 \leq a_{i}<p$, formam uma base para FG. Além disso, estes elementos, exceto $\eta(0,0, \ldots, 0)=1$, constituem uma base de $\Delta(G)$.

Dada uma sequência $G=H_{1}>H_{2}>\cdots>H_{n+1}=\langle 1\rangle$, como no enunciado do Lema 2.2.13, obtemos uma base para FG. Além disso, para cada elemento da base

$$
\eta=\eta\left(a_{1}, a_{2}, \ldots, a_{n}\right)=\left(x_{1}-1\right)^{a_{1}}\left(x_{2}-1\right)^{a_{2}} \cdots\left(x_{n}-1\right)^{a_{n}},
$$

com $0 \leq a_{i}<p$, associamos seu peso

$$
\omega(\eta)=a_{1} \nu\left(x_{1}\right)+a_{2} \nu\left(x_{2}\right)+\cdots+a_{n} \nu\left(x_{n}\right) .
$$

Suponha que $\left\{G_{i}\right\}$ e $\left\{H_{i}\right\}$ sejam duas $N_{p}$-sequências em $G$. Então escrevemos $\left\{G_{i}\right\} \leq$ $\left\{H_{i}\right\}$ se $G_{i} \subseteq H_{i}$, para todo $i \in \mathbb{N}$ e dizemos que $\left\{G_{i}\right\}$ é menor do que $\left\{H_{i}\right\}$.

Teorema 2.2.14 (Teorema III.3.7, [29]). Seja F um corpo de característica p e G um p-grupo finito. Então a sequência $\left\{D_{t}(F G)\right\}$ dos subgrupos dimensionais é a menor $N_{p}$-sequência de 
G. Além disso, a filtração de $\Delta(G)$ determinada por $\left\{D_{t}(F G)\right\}$ é precisamente $\left\{\Delta(G)^{t}\right\}$, a filtração de potências do ideal de aumento.

Note que como $G$ é um p-grupo finito, o ideal de aumento é nilpotente e assim a cadeia (2.2) é finita.

Entre todas as $N_{p}$-sequências de um p-grupo finito $G$, existe uma sequência conhecida como $\Gamma$-sequência, definida por: $\Gamma_{1}=G$ e $\Gamma_{i}=\left\langle\left(\Gamma_{i-1}, G\right), \Gamma_{\lceil i / p\rceil}^{p}\right\rangle$. Na verdade pode-se provar que a $\Gamma$-sequência é igual à $N_{p}$-sequência $\left\{D_{t}(F G)\right\}$ dos subgrupos dimensionais de $G$, como em [20, Teorema 5.5]. 


\section{Capítulo 3}

\section{Propriedades de Lie e involuções}

\section{clássicas orientadas}

Neste capítulo caracterizamos as álgebras de grupo $F G$, onde $G$ é um grupo sem elementos de ordem 2, tais que o conjunto dos elementos simétricos ou anti-simétricos sob a involução clássica orientada é Lie $n$-Engel ou Lie nilpotente .

\subsection{Preliminares}

Para um primo $p$ dado, um elemento do grupo $G$ é chamado um $p$-elemento se sua ordem é finita e uma potência de $p$ e é chamado um $p^{\prime}$-elemento se é de ordem finita não divisível por $p$. Para um grupo $G$, denotamos por $\zeta=\zeta(G)$ o seu centro.

Denotamos por $Q_{8}=\left\langle x, y: x^{4}=1, x^{2}=y^{2}, x^{y}=x^{-1}\right\rangle$ o grupo quatérnio de ordem 8. Lembramos que $N$ denota o núcleo da orientação $\sigma$.

Lema 3.1.1. Se $(F G)^{+}$é Lie $n$-Engel, para algum $n$, e car $(F) \neq 2$, então todo elemento de ordem 2 em $N$ é central.

Demonstração. Suponha que a característica de $F$ seja $p>2$. Seja $g \in N$ tal que $g^{2}=1$. Consideraremos vários casos.

Tomemos primeiro $x \in N$ tal que $o(x)=2$. Podemos escolher um $m$ tal que $p^{m}$ é maior que $n$ e assim $0=[g, \underbrace{x, \ldots, x}_{p^{m}}]=\left[g, x^{p^{m}}\right]$. Como $p$ é ímpar, $x^{p^{m}}=x$ e, em consequência, $g$ 
comuta com $x$. Agora suponha $x \in N$ tal que $x^{2} \neq 1$. Logo

$$
0=[x+x^{-1}, \underbrace{g, \ldots, g}_{p^{m}}]=\left[x+x^{-1}, g^{p^{m}}\right]=\left[x+x^{-1}, g\right] .
$$

Assim, $x g$ é igual a $g x$ ou $g x^{-1}$. Agora se $x g=g x$ não há nada que provar. Se $x g=g x^{-1}$, então $(x g)^{2}=x g g x^{-1}=1$ e assim, pelo primeiro caso, $g$ e $x g$ comutam, donde $g x=x g$.

Agora, suponha $x \in G \backslash N$ tal que $x^{2} \neq 1$. Então

$$
0=[x-x^{-1}, \underbrace{g, \ldots, g}_{p^{m}}]=\left[x-x^{-1}, g^{p^{m}}\right]=\left[x-x^{-1}, g\right] .
$$

Assim, $x g=g x$ ou $x g=x^{-1} g$. Como o segundo caso leva a uma contradição, então, também ocorre $x g=g x$.

Finalmente, se $x \in G \backslash N \operatorname{com} x^{2}=1$, então $x g \in G \backslash N$. Se $(x g)^{2}=1$, então $x g=$ $(x g)^{-1}=g^{-1} x^{-1}=g x$. Desta forma podemos supor $(x g)^{2} \neq 1$, logo, pelo caso anterior, $x g$ comuta com $g$ e, como antes, $x$ e $g$ comutam.

Se a característica de $F$ é zero, então $\mathbb{Z} G \subseteq F G$. Assim, $(\mathbb{Z} G)^{+}$é Lie $n$-Engel e assim $((\mathbb{Z} / 3 \mathbb{Z}) G)^{+}$é Lie $n$-Engel. Usando a conclusão obtida no caso em que a característica é diferente de zero, todos os elementos de ordem 2 em $N$ são centrais em $G$.

Usaremos o seguinte lema em várias oportunidades.

Lema 3.1.2. Sejam $g$ e $h$ elementos de $G$ tais que $g^{2} \neq 1$ e $h^{2} \neq 1$. Então valem as seguintes propriedades:

(i) $S e\left[g+g^{-1}, h+h^{-1}\right]=0$, então

(a) $g h \in\left\{h g, h^{-1} g, h g^{-1}\right\}$ ou

(b) $\left(g^{\alpha} h^{\beta}\right)^{2}=1$, para todos $\alpha, \beta \in\{-1,1\}$.

(ii) $S e\left[g-g^{-1}, h-h^{-1}\right]=0$, então

(a) $g h=h g$ ou

(b) $\left(g^{\alpha} h^{\beta}\right)^{2}=1$, para todos $\alpha, \beta \in\{-1,1\}$. 
(iii) $S e\left[g-g^{-1}, h+h^{-1}\right]=0$, então

(a) $g h \in\left\{h g, h^{-1} g\right\}$ ou

(b) $o(g)=4=o(h)$ e $g^{2}=h^{2}$.

Demonstração.

(i) Suponha $\left[g+g^{-1}, h+h^{-1}\right]=0$. Isto implica

$$
g h+g h^{-1}+g^{-1} h+g^{-1} h^{-1}=h g+h g^{-1}+h^{-1} g+h^{-1} g^{-1} .
$$

Se $\operatorname{car}(F) \neq 3$, então $g h=h g, g h=h g^{-1}, g h=h^{-1} g$ ou $g h=h^{-1} g^{-1}$. Suponha $g h \notin\left\{h g, h^{-1} g, h g^{-1}\right\}$, então $g h=h^{-1} g^{-1}$, isto é, $(g h)^{2}=1$ o que é equivalente a $\left(g^{-1} h^{-1}\right)^{2}=1$. Logo, de (3.1) temos $g h^{-1}+g^{-1} h=h g^{-1}+h^{-1} g$. Desta forma $g h^{-1}=$ $h g^{-1}$ ou $g h^{-1}=h^{-1} g$ e assim $g h=h g$, o que não pode ocorrer. Em consequência, $\left(g h^{-1}\right)^{2}=1$ é equivalente a $\left(g^{-1} h\right)^{2}=1$.

Se $\operatorname{car}(F)=3$, em (3.1) devemos considerar outras possibilidades: $g h=g h^{-1}, g h=$ $g^{-1} h$ ou $g h^{-1}=g^{-1} h^{-1}$. Não obstante, todos estes casos levam a uma contradição.

(ii) Suponha $\left[g-g^{-1}, h-h^{-1}\right]=0$. Temos então

$$
g h+g^{-1} h^{-1}+h g^{-1}+h^{-1} g=h g+h^{-1} g^{-1}+g h^{-1}+g^{-1} h .
$$

Se $\operatorname{car}(F) \neq 3$, então $g h=h g, g h=h^{-1} g^{-1}, g h=g h^{-1}$ ou $g h=g^{-1} h$, mas dado que $g^{2} \neq 1, h^{2} \neq 1$, temos $g h=h g$ ou $(g h)^{2}=1$. Suponha $(g h)^{2}=1$. Assim, de (3.2), temos $h g^{-1}+h^{-1} g=g h^{-1}+g^{-1} h$. Desta forma $h g^{-1}=g h^{-1}$ ou $h g^{-1}=g^{-1} h$, o que implica $\left(g h^{-1}\right)^{2}=1$ ou $g h=h g$ e o resultado segue.

Suponha $\operatorname{car}(F)=3$, em (3.2) devemos considerar outras possibilidades: $g h=g^{-1} h^{-1}=$ $h g^{-1}, g h=g^{-1} h^{-1}=h^{-1} g, g h=h g^{-1}=h^{-1} g$ ou $g^{-1} h^{-1}=h g^{-1}=h^{-1} g$.

Se $g h=g^{-1} h^{-1}=h g^{-1}$, então $h^{-1} g^{-1}=h g=g h^{-1}$ e assim de (3.2), $h^{-1} g=g^{-1} h$, isto implica $g h^{-1}=h g^{-1}$ e podemos concluir que $g h=h g$. 
Se $g h=g^{-1} h^{-1}=h^{-1} g$, então $h^{-1} g^{-1}=h g=g^{-1} h$ e de $(3.2) h g^{-1}=g h^{-1}$ e assim $g^{-1} h=h^{-1} g$. Isto, junto as equações anteriores, implica $g h=h g$.

Se $g h=h g^{-1}=h^{-1} g$, então $h^{-1} g^{-1}=g h^{-1}=g^{-1} h$ e assim de (3.2), $g^{-1} h^{-1}=h g$ e assim $h^{-1} g^{-1}=g h$. Logo $g h=g h^{-1}$, o que é uma contradição, pois $h^{2} \neq 1$.

Finalmente, se $g^{-1} h^{-1}=h g^{-1}=h^{-1} g$, então $h g=g h^{-1}=g^{-1} h$ e assim, de (3.2), $g h=h^{-1} g^{-1}, \operatorname{logo} h g=g^{-1} h^{-1}$. Daí $h g=h g^{-1}$, que é uma contradição, pois $g^{2} \neq 1$.

(iii) Assuma $\left[g-g^{-1}, h+h^{-1}\right]=0$, donde

$$
g h+g h^{-1}+h g^{-1}+h^{-1} g^{-1}=h g+h^{-1} g+g^{-1} h+g^{-1} h^{-1} .
$$

Se $\operatorname{car}(F) \neq 3$, de $(3.3)$ e dado que $g^{2} \neq 1$, temos $g h=h g, g h=h^{-1} g$ ou $g h=g^{-1} h^{-1}$. Suponha $g h=g^{-1} h^{-1}$, isto é, $g^{2}=h^{-2}$. De (3.3), $g h^{-1}+h g^{-1}=h^{-1} g+g^{-1} h$. Logo $g h^{-1}=h^{-1} g$ ou $g h^{-1}=g^{-1} h$. A segunda opção implica $g^{2}=h^{2}$, e assim $o(g)=4$. A primeira opção implica $g h=h g$. De onde segue o resultado.

Suponha $\operatorname{car}(F)=3$. Assim, em (3.3), além das possibilidades anteriores, devemos considerar os seguintes casos: $g h=g h^{-1}$ ou $h g^{-1}=h^{-1} g^{-1}$. Pelas hipóteses, em qualquer caso obtemos uma contradição.

Corolário 3.1.3. Suponha $\operatorname{car}(F)=p>2$ e $(F G)^{+}$Lie $p^{m}$-Engel para algum $m \geq 1$. Sejam $g$ e $h$ elementos de $G$ tais que $g^{2} \neq 1$ e $h^{2 p^{m}} \neq 1$. Se $\sigma(g)=\sigma(h)=-1$, então $\left(g, h^{p^{m}}\right)=1$.

Demonstração. Dado que $(F G)^{+}$é Lie $p^{m}$-Engel, obtemos $\left[g-g^{-1}, h^{p^{m}}-h^{-p^{m}}\right]=0$. Assim, pela parte (ii) do Lema 3.1.2, temos $\left(g, h^{p^{m}}\right)=1$ ou $\left(g h^{p^{m}}\right)^{2}=1$. A segunda opção e o Lema 3.1.1 implicam que $g h^{p^{m}}$ é central. Assim, novamente obtemos $\left(g, h^{p^{m}}\right)=1$.

No estudo das propriedades de Lie nilpotência e Lie $n$-Engel em $(F G)^{+}$sob a involução clássica, foi importante estudar o caso em que $G=Q_{8}$. O seguinte lema mostra que $\left(F Q_{8}\right)^{+}$, sob uma involução clássica orientada, é Lie $n$-Engel (Lie nilpotente) unicamente quando a orientação é trivial. 
Lema 3.1.4. Sejam FG uma álgebra de grupo com $\operatorname{car}(F) \neq 2$ e $G$ um grupo tal que $Q_{8} \subseteq G$. Seja $\sigma$ uma orientação não trivial de $G$. Se $(F G)^{+}$é Lie n-Engel, para algum $n \in \mathbb{N}$, então $Q_{8} \subseteq N$.

Demonstração. Seja $Q_{8}=\left\langle x, y: x^{4}=1, x^{2}=y^{2}, x^{y}=x^{-1}\right\rangle$ o grupo quatérnio de 8 elementos. Sabemos que $\left(F Q_{8}\right)^{+}$sob a involução clássica, isto é, quando $\sigma(x)=\sigma(y)=1$, é comutativo. Consideraremos as diferentes possibilidades para uma orientação $\sigma$ não trivial em $Q_{8}$.

Se $\sigma(x)=1$ e $\sigma(y)=-1$, então $x y-(x y)^{-1}$ e $y-y^{-1}$ são elementos simétricos. Calculamos

$$
\begin{aligned}
& {\left[x y-(x y)^{-1}, y-y^{-1}\right]=-4\left(x-x^{-1}\right) ;} \\
& {\left[x y-(x y)^{-1}, y-y^{-1}, y-y^{-1}\right]=-4^{2}\left(x y-(x y)^{-1}\right) ;} \\
& {[x y-(x y)^{-1}, \underbrace{y-y^{-1}, \ldots, y-y^{-1}}_{3 \text { vezes }}]=4^{3}\left(x-x^{-1}\right) ;} \\
& {[x y-(x y)^{-1}, \underbrace{y-y^{-1}, \ldots, y-y^{-1}}_{4 \text { vezes }}]=4^{4}\left(x y-(x y)^{-1}\right) .}
\end{aligned}
$$

Então, para qualquer $k \in \mathbb{Z}^{+}$, obtemos

$$
[x y-(x y)^{-1}, \underbrace{y-y^{-1}, \ldots, y-y^{-1}}_{k \text { vezes }}]= \begin{cases}(-1)^{\left(\frac{k+1}{2}\right)} 4^{k}\left(x-x^{-1}\right), & \text { se } k \text { é ímpar } \\ (-1)^{\left(\frac{k}{2}\right)} 4^{k}\left(x y-(x y)^{-1}\right), & \text { se } k \text { é par }\end{cases}
$$

Assim $[x y-(x y)^{-1}, \underbrace{y-y^{-1}, \ldots, y-y^{-1}}_{k \text { vezes }}] \neq 0$, para qualquer $k$, pois a $\operatorname{car}(F) \neq 2$.

O caso $\sigma(x)=-1$ e $\sigma(y)=1$ é similar ao anterior. Agora, assumimos $\sigma(x)=\sigma(y)=-1$.

Como $x_{0}=x y$ e $y$ geram $Q_{8}$ e $\sigma\left(x_{0}\right)=1, \sigma(y)=-1$, o resultado segue como antes.

Portanto, $\left(F Q_{8}\right)^{+}$não é Lie $n$-Engel, para qualquer $n \in \mathbb{Z}^{+}$e qualquer orientação não trivial.

Primeiro estudaremos a Lie nilpotência de $(F G)^{+}$e $(F G)^{-}$quando o conjunto $\zeta(G)^{2}=\left\{z^{2}: z \in \zeta(G)\right\}$ é infinito.

Lema 3.1.5. Seja $G$ um grupo tal que $\left|\zeta(G)^{2}\right|=\infty$. Se $\alpha \in F G$ tal que $\left(\sigma(z) z^{2}-1\right) \alpha=0$, para todo $z \in \zeta(G)$, então $\alpha=0$. 
Demonstração. Escrevemos $\alpha=\sum \alpha_{i} x_{i}$ onde $\alpha_{i} \in F \zeta^{2}$ e os elementos $x_{i}$ estão numa transversal direita de $\zeta^{2}$ em $G$ tal que $x_{1}=1$. Então $\left(\sigma(z) z^{2}-1\right) \alpha=0$ implica $\left(\sigma(z) z^{2}-\right.$ 1) $\alpha_{1}=0$. Isto significa $\sigma(z) z^{2} \alpha_{1}=\alpha_{1}$ para um número infinito de elementos $z^{2} \in \zeta(G)^{2}$, logo temos $\alpha_{1}=0$.

Multiplicando $\alpha$ por elementos adequados, segue que $\alpha_{i}=0$, para todo $i$ e, portanto, $\alpha=0$.

Proposição 3.1.6. Seja $G$ um grupo tal que $\left|\zeta(G)^{2}\right|=\infty$. Então $(F G)^{+}$ou $(F G)^{-}$é Lie nilpotente de indice $n$ se, e somente se, FG é Lie nilpotente de indice $n$.

Demonstração. Dado que $(F G)^{+}$é Lie nilpotente de índice $n \in \mathbb{Z}^{+}, F G$ satisfaz a *identidade polinomial

$$
f\left(x_{1}, \ldots, x_{n}\right)=\left[x_{1}+x_{1}^{*}, \ldots, x_{n}+x_{n}^{*}\right]=0 .
$$

Para um índice fixo $i \in\{1, \ldots, n\}$, escrevemos $f=f_{1}+f_{2}$ onde $f_{1}$ é a soma de todos os monômios de $f$ que contêm $x_{i}$. Então, se $z_{i} \in \zeta(G)$,

$$
f\left(x_{1}, \ldots, z_{i} x_{i}, \ldots, x_{n}\right)=z_{i} f_{1}+z_{i}^{*} f_{2}
$$

é uma $*$-identidade polinomial para $F G$. Também $z_{i}{ }^{*} f=z_{i}{ }^{*} f_{1}+z_{i}{ }^{*} f_{2}$ é uma $*$-identidade polinomial para $F G$. Logo, $z_{i} f_{1}-z_{i}^{*} f_{1}=\left(z_{i}-z_{i}^{*}\right) f_{1}$ é uma $*$-identidade polinomial para $F G$. Agora, como $z_{i}^{*}=\sigma\left(z_{i}\right) z_{i}^{-1}$, obtemos que $\left(\sigma\left(z_{i}\right) z_{i}^{2}-1\right) f_{1}$ é uma identidade polinomial para FG. Uma aplicação reiterada deste argumento nos leva à seguinte identidade polinomial para $F G$

$$
\left(\sigma\left(z_{1}\right) z_{1}^{2}-1\right)\left(\sigma\left(z_{2}\right) z_{2}^{2}-1\right) \cdots\left(\sigma\left(z_{n}\right) z_{n}^{2}-1\right) \tau
$$

onde $\tau=\left[x_{1}, \ldots, x_{n}\right]$. Dado que $\left|\zeta(G)^{2}\right|=\infty$, pelo Lema 3.1.5, obtemos que $\tau$ é uma identidade polinomial para $F G$ e, portanto, $F G$ é Lie nilpotente de índice $n$.

Se $(F G)^{-}$é Lie nilpotente de índice $n$, então $F G$ satisfaz a $*$-identidade polinomial

$$
h\left(x_{1}, \ldots, x_{n}\right)=\left[x_{1}-x_{1}^{*}, \ldots, x_{n}-x_{n}^{*}\right]=0
$$


e a prova segue de forma similar.

A recíproca é trivial.

No que segue, por

$$
D_{k}=\left\langle x, y: x^{k}=1, y^{2}=1,(x y)^{2}=1\right\rangle
$$

denotaremos o grupo diedral de ordem $2 k$ e, por

$$
D_{\infty}=\left\langle x, y: y^{2}=1,(x y)^{2}=1\right\rangle
$$

\section{o grupo diedral infinito.}

Lema 3.1.7. Seja $G=\langle a, b\rangle$ o grupo gerado pelos elementos a e $b$ tais que $b^{-1} a b=a^{-1}$. Se $(F G)^{+}$é Lie $n$-Engel, para algum $n \in \mathbb{N}$, então vale uma das seguintes condições:

(i) $a^{2}=1$ (e Gé abeliano) ou

(ii) $\sigma(a)=1, \sigma(b)=-1, b^{2 p^{m}}=1$, para algum $m>0 e\left\langle a, b^{p^{m}}\right\rangle \simeq D_{k}$, onde $k=o(a)$ se $o(a)$ é finito ou $k=\infty$, caso contrário.

Demonstração. Se $a^{2}=1$, não há nada a provar. Assim, podemos assumir $a^{2} \neq 1$. Note que, para qualquer índice $i$, temos $b^{-1} a^{i} b=a^{-i}$, logo $a^{i} \in \zeta(G)$ se, e somente se, $a^{2 i}=1$. Suponha $\operatorname{car}(F)=p>2$. É fácil verificar as igualdades:

$$
b^{-i} a b^{i}=\left\{\begin{array}{l}
a^{-1}, \text { se } i \text { é ímpar } \\
a, \text { se } i \text { é par. }
\end{array}\right.
$$

Seja $m>0$ tal que $p^{m}$ é maior que $n$. Consideraremos três casos separadamente, dependendo dos valores de $\sigma$ sobre $G$.

1. $\sigma(a)=-1$ e $\sigma(b)=1$.

Primeiro assumimos que $b$ tem ordem finita. Dado que $b^{2} \in \zeta(G)$, mas $b \notin \zeta(G)$,o $o(b)$ é da forma $2 k$ para algum $k \in \mathbb{Z}^{+}$. Como $b \in N$, se $k$ fosse ímpar, então do Lema 3.1.1 
$b^{k}$ seria central e assim $b$ também seria central. Logo, 4 divide a ordem de $b$ e podemos escrever $o(b)=2^{r} s$, com $s$ um inteiro ímpar e $r \geq 2$.

Como $0=\left[a-a^{-1}, b^{p^{m} s}+b^{-p^{m} s}\right]$, da parte (iii) do Lema 3.1.2, temos

$$
a b^{p^{m} s}=b^{p^{m} s} a, \quad a b^{p^{m} s}=b^{-p^{m} s} a \quad \text { ou } \quad o(a)=4=o\left(b^{p^{m} s}\right) \text { e } a^{2}=b^{2 p^{m} s} .
$$

A primeira opção implica $a^{2}=1$, uma contradição.

Se a segunda opção é válida, temos $a^{2}=b^{2 p^{m} s}$. Então $a^{2} \in \zeta(G)$ e assim $a^{4}=1$. Desta forma as duas últimas opções implicam $o(a)=4, b^{4 p^{m} s}=1$ e $a^{2}=b^{2 p^{m} s}$. Assim, $o(b)=4 s . \operatorname{Logo} o(a)=o\left(b^{s}\right)=4$ e $a^{2}=b^{2 s}$, isto é, $\left\langle a, b^{s}\right\rangle \simeq Q_{8}$. Como $\sigma(a)=-1$ e, pelo Lema 3.1.4, $Q_{8} \subseteq N$, temos uma contradição. Se a ordem de $b$ é infinita, podemos escrever $0=\left[a-a^{-1}, b^{p^{m}}+b^{-p^{m}}\right]$ e, novamente da parte (iii) do Lema 3.1.2, obtemos

$$
a b^{p^{m}}=b^{p^{m}} a, \text { ou } a b^{p^{m}}=b^{-p^{m}} a .
$$

Como acima, a segunda opção implica que $b$ tem ordem finita, então $a^{2}=1$, uma contradição.

2. $\sigma(a)=1$ e $\sigma(b)=-1$.

Assumimos $b^{2 p^{m}} \neq 1$. Então $(a b)^{2} \neq 1$ e $\left[a b-(a b)^{-1}, b^{p^{m}}-b^{-p^{m}}\right]=0$. Usando o Corolário 3.1.3, temos $a b^{p^{m}}=b^{p^{m}} a$ e obtemos $a^{2}=1$, uma contradição. Logo, devemos ter $b^{2 p^{m}}=1$ e assim $\left\langle a, b^{p^{m}}\right\rangle$ é isomorfo a $D_{k}$ ou $D_{\infty}$ dependendo da ordem de $a$.

3. $\sigma(a)=\sigma(b)=-1$.

Seja $c=a b$. Então $\sigma(c)=1$ e $c^{-1} a c=a^{-1}$. Logo temos as mesmas condições do primeiro caso, o qual já provamos que leva a uma contradição.

Portanto, acabamos de provar as igualdades $a^{2}=1$ ou $b^{2 p^{m}}=1$, para algum $m \geq 0$. Também, se $a^{2} \neq 1$, a única possibilidade para $\sigma$ é $\sigma(a)=1$ e $\sigma(b)=-1$.

Se $\operatorname{car}(F)=0$, então $\mathbb{Z} G \subseteq F G$. Logo $(\mathbb{Z} G)^{+}$é Lie $n$-Engel e assim, para qualquer primo $\operatorname{ímpar} q,((\mathbb{Z} / q \mathbb{Z}) G)^{+}$é Lie $n$-Engel. Usando o resultado anterior, $a^{2}=1$ ou $b^{2 q^{m}}=1$, para 
algum $m \geq 0$. Se $a^{2} \neq 1$, então a última opção é satisfeita para todo primo ímpar $q$. Assim, $b^{2}=1$ o que implica $a^{2}=1$, uma contradição. Portanto, $a^{2}=1$ e $G$ é abeliano.

\subsection{Grupos sem elementos de ordem 2}

Seja $G$ um grupo sem elementos de ordem 2. Lembramos que um grupo $G$ é dito $p$ abeliano se $G^{\prime}$, o subgrupo comutador de $G$, é um $p$-grupo finito e 0-abeliano significa abeliano. Nesta seção provaremos que se $(F G)^{+}$ou $(F G)^{-}$é Lie $n$-Engel, para algum $n$ (Lie nilpotente), então $F G$ é Lie $m$-Engel, para algum $m$ (Lie nilpotente).

Para um subgrupo normal $H$ de $G$ denotamos por $\Delta(G, H)$ o núcleo da aplicação natural $\varepsilon_{H}: F G \rightarrow F(G / H)$ definida por:

$$
\varepsilon_{H}\left(\sum_{g \in G} \alpha_{g} g\right)=\sum_{g \in G} \alpha_{g} g H
$$

e $\Delta(G)=\Delta(G, G)$, veja Proposição 2.2.8.

Lema 3.2.1. Suponha que $G$ é um grupo sem elementos de ordem 2 e $\operatorname{car}(F)=p>2$. Suponha $(F G)^{+}$ou $(F G)^{-}$Lie nilpotente. Se o centro de $G$ tem um elemento de ordem infinita ou um p'-elemento não trivial, então $G$ é p-abeliano.

Demonstração. Seja z um elemento central cuja ordem é infinita ou finita relativamente prima com $p$. Se $o(z)=\infty$, então $\left|\zeta(G)^{2}\right|=\infty$ e o resultado segue da Proposição 3.1.6.

Logo, podemos supor que $z$ seja de ordem ímpar relativamente prima com $p$. Em particular, $\sigma(z)=1$. Como $(F G)^{+}$é Lie nilpotente, da demonstração da Proposição 3.1.6, temos $\left(z^{2}-1\right)^{n}[R, R, \ldots, R]=0$, para algum $n \geq 0$, onde $R=F G$. Seja $A=\left(z^{2}-1\right)^{n} R$. Então $[A, R, \ldots, R]=\left(z^{2}-1\right)^{n}[R, R, \ldots, R]=0$ e, do Teorema em [14], temos $[A, R] R=$ $\left(z^{2}-1\right)^{n}[R, R] R$ um ideal nilpotente de $R$. Assim, $\left(1-z^{2}\right)^{n} \Delta\left(G, G^{\prime}\right)$ é um ideal nilpotente. Logo, também $\left(1-z^{2}\right) \Delta\left(G, G^{\prime}\right)$ é nilpotente. Então $\left(1-z^{2}\right)^{m} \Delta\left(G, G^{\prime}\right)^{m}=0$, para algum $m>0$. Desta forma, para todo $\alpha \in \Delta\left(G, G^{\prime}\right)^{m}$, temos $\left(1-z^{2}\right)^{m} \alpha=0$.

Seja $H=\left\langle z^{2}\right\rangle$. Então, para todo $\alpha \in \Delta\left(G, G^{\prime}\right)^{m}$, obtemos $\left(1-z^{2}\right)^{m} \alpha=\left(1-z^{2}\right)\left(1-z^{2}\right)^{m-1} \alpha=0$ e $\operatorname{assim}\left(1-z^{2}\right)^{m-1} \alpha \in A n n_{r}(\Delta(G, H))=F G \cdot \widehat{H}$, pelo Lema 3.4.3 em [30]. Logo, $\left(1-z^{2}\right)^{m-1} \alpha=\beta \widehat{H}$, para algum $\beta \in F G$. Como $z^{2}$ é um 
$p^{\prime}$-elemento, $|H|$ não é divisível por $p$. Assim, $\left(1-z^{2}\right)^{m-1} \alpha \widehat{H} /|H|=\beta \widehat{H} \widehat{H} /|H|=\beta \widehat{H}$. Desta forma, $\left(1-z^{2}\right)^{m-1} \alpha=0$. Portanto, podemos provar a igualdade, $\left(1-z^{2}\right)^{m-1} \Delta\left(G, G^{\prime}\right)^{m}=0$.

Repetindo o argumento anterior, chegamos à igualdade $\left(1-z^{2}\right) \Delta\left(G, G^{\prime}\right)^{m}=0$. Seja $x$ um elemento qualquer de $G^{\prime}$. Então $\left(1-z^{2}\right)(1-x)^{p^{m}}=0$. Donde, $\left(1-z^{2}\right)\left(1-x^{p^{m}}\right)=0$. Desta forma, $1+z^{2} x^{p^{m}}=x^{p^{m}}+z^{2}$. Em consequência, $x^{p^{m}}=1$ e, portanto, $x$ é um $p$-elemento. Logo $G^{\prime}$ é um $p$-grupo. Afirmamos que $\Delta\left(G^{\prime}\right)^{m}=0$. De fato, temos $\left(1-z^{2}\right) \Delta\left(G^{\prime}\right)^{m}=0$ e daí $\Delta\left(G^{\prime}\right)^{m}=z^{2} \Delta\left(G^{\prime}\right)^{m}$. Agora, dado $\alpha \in \Delta\left(G^{\prime}\right)^{m}$ arbitrário, existe $\beta \in \Delta\left(G^{\prime}\right)^{m}$, tal que $\alpha=z^{2} \beta$. Assim para $x \in \operatorname{supp}(\alpha)$, existe $y \in \operatorname{supp}(\beta)$ tal que $x=z^{2} y$. Mas, como $z^{2}$ é um $p^{\prime}$ elemento e $G^{\prime}$ um $p$-grupo, chegamos à uma contradição. Desta forma, $\alpha=0$, donde $\Delta\left(G^{\prime}\right)^{m}=0$. Pelo [30, Teorema 6.3.1 ], $G^{\prime}$ é um p-grupo finito, como queríamos provar.

Lema 3.2.2. Seja $H=\langle a, b\rangle$ o grupo gerado por dois elementos $a, b$ tais que $a \neq 1 e$ $b^{-1} a b=a^{-1}$. Suponha que $H$ não tem elementos de ordem 2. Então nem $(F H)^{+}$e nem $(F H)^{-}$são Lie n-Engel.

A prova segue imediatamente do Lemma 3.1.7.

Lema 3.2.3. Seja $H=\langle g, h\rangle$ com $\left[g+c_{1} g^{-1}, h+c_{2} h^{-1}\right]=0$, para alguns $c_{1}, c_{2} \in F$. Suponha que $H$ não contenha elementos de ordem 2. Se $(F H)^{+}$ou $(F H)^{-}$é Lie n-Engel, então $H$ é abeliano.

Demonstração. Assuma $[g, h] \neq 0$. Então $g, h, g h \neq 1$ e $g h \neq h g, g^{-1} h, g h^{-1}, h^{-1} g^{-1}$. Além disso, pelo Lema 3.2.2, $g h \neq h g^{-1}, h^{-1} g$. Assim, a igualdade $\left[g+c_{1} g^{-1}, h+c_{2} h^{-1}\right]=0$ implica $g h=g^{-1} h^{-1}$ e donde $g^{2}=h^{-2}$. Desta forma, $g^{2} \in \zeta(H)$. Como $g \notin \zeta(H)$, então $g$ tem ordem infinita. Além disso,

$$
\begin{aligned}
0=\left[g+c_{1} g^{-1}, h+c_{2} h^{-1}\right] & =\left[\left(1+c_{1} g^{-2}\right) g,\left(1+c_{2} h^{-2}\right) h\right] \\
& =\left(1+c_{1} g^{-2}\right)\left(1+c_{2} h^{-2}\right)[g, h] .
\end{aligned}
$$

Como $C=\left\langle g^{2}\right\rangle$ é um grupo cíclico infinito, pelo Lema 2.2.3, o elemento $\left(1+c_{1} g^{-2}\right)\left(1+c_{2} h^{-2}\right)$ não é um divisor de zero em $F C$ e, pelo Lema 2.2 .2 , não pode ser um divisor de zero em $F H$. Portanto, $[g, h]=0$, o que é uma contradição. 
Lema 3.2.4. Seja $G$ um grupo sem elementos de ordem 2 e $\operatorname{car}(F) \neq 2$. Suponha $(F G)^{+}$ ou $(F G)^{-}$Lie $n$-Engel, para algum $n \in \mathbb{N}$. Então,

(i) se $\operatorname{car}(F)=0$, então $G$ é abeliano;

(ii) se $\operatorname{car}(F)=p>0$, então $G^{p^{m}} \subseteq \zeta(G)$, para algum $m>0$.

Demonstração. Assuma $(F G)^{+}$Lie $n$-Engel, para algum $n \in \mathbb{N}$. Suponha $\operatorname{car}(F)=p>2$. Sejam $m>0$ tal que $p^{m} \geq n$ e $h \in G$ um elemento fixo. Então, para todo $g \in G$, tem-se

$$
[g+\sigma(g) g^{-1}, \underbrace{h+\sigma(h) h^{-1}, \ldots, h+\sigma(h) h^{-1}}_{p^{m} \text { vezes }}]=0 .
$$

Assim, $\left[g+\sigma(g) g^{-1}, h^{p^{m}}+\sigma(h) h^{-p^{m}}\right]=0$. O Lema 3.2 .3 implica $g h^{p^{m}}=h^{p^{m}} g$. Assim $G^{p^{m}} \subseteq \zeta(G)$

Se $\operatorname{car}(F)=0$, então $\mathbb{Z} G \subseteq F G$. Assim, $(\mathbb{Z} G)^{+}$é Lie $n$-Engel donde, para qualquer primo $q$, tem-se que $((\mathbb{Z} / q \mathbb{Z}) G)^{+}$é Lie $n$-Engel. Usando o resultado anterior, $G^{q^{m}} \subseteq \zeta(G)$, para algum $m>0$. Dado que isto é válido para todo primo ímpar $q$, então $G$ é abeliano.

Se $(F G)^{-}$é Lie $n$-Engel, para algum $n$, a prova segue de forma similar.

Agora podemos provar os principais resultados deste capítulo.

Teorema 3.2.5. Sejam F um corpo de característica $p \neq 2, G$ um grupo sem elementos de ordem 2 e $\sigma$ uma orientação não trivial de $G$. Então $(F G)^{+}\left(\right.$ou $\left.(F G)^{-}\right)$é Lie n-Engel, para algum $n \in \mathbb{N}$, se, e somente se, FG é Lie $m$-Engel, para algum $m \in \mathbb{N}$.

Demonstração. Assuma $(F G)^{+}$Lie $n$-Engel, para algum $n \in \mathbb{N}$. Se car $(F)=0$, o resultado já foi provado no Lema 3.2.4.

Suponha $\operatorname{car}(F)=p>2$. Sabemos que

$$
[x_{1}+x_{1}^{*}, \underbrace{x_{2}+x_{2}^{*}, \ldots, x_{2}+x_{2}^{*}}_{n \text { vezes }}]=0
$$

é uma *-identidade polinomial para $F G$. Então, de [17, p. 196], FG satisfaz uma identidade polinomial. 
Necessitamos provar que $G$ é nilpotente; logo, é suficiente mostrar que $G / \zeta(G)$ é nilpotente. Do Lema 3.2.4, temos $G^{p^{m}} \subseteq \zeta(G)$, para algum $m>0$, assim $G / \zeta(G)$ é um $p$-grupo de expoente limitado. Do Lema 3.2.7 em [24], obtemos que G é nilpotente e [24, Proposição 1.3.7] mostra que $G^{\prime}$ é um p-grupo de expoente limitado. Também, por [29, Teorema 5.2.14], o FC-subgrupo $\phi=\phi(G)$ de $G$ é de índice finito em $G$ e $\left|\phi^{\prime}\right|<\infty$. Mas $\zeta(G) \subseteq \phi$ e $G / \zeta(G)$ é um $p$-grupo. Assim, $G / \phi$ e $\phi^{\prime} \subseteq G^{\prime}$ são ambos $p$-grupos finitos. Logo, do Teorema 2.1.3, $F G$ é Lie $m$-Engel, para algum $m \in \mathbb{N}$.

Se $(F G)^{-}$é Lie $n$-Engel, para algum $n \in \mathbb{N}$, então

$$
[x_{1}-x_{1}^{*}, \underbrace{x_{2}-x_{2}^{*}, \ldots, x_{2}-x_{2}^{*}}_{n \text { vezes }}]=0
$$

é uma *-identidade polinomial e a prova é similar.

A recíproca é trivial.

Teorema 3.2.6. Sejam $F$ um corpo de característica $p \neq 2, G$ um grupo sem elementos de ordem 2 e $\sigma$ uma orientação não trivial de $G$. Então $(F G)^{+}\left(\right.$ou $\left.(F G)^{-}\right)$é Lie nilpotente se, e somente se, FG é Lie nilpotente.

Demonstração. Assuma $(F G)^{+}\left(\right.$ou $\left.(F G)^{-}\right)$Lie nilpotente. De novo, se car $(F)=0$, o resultado segue do Lema 3.2.4.

Suponha $\operatorname{car}(F)=p>2$. Do Teorema 3.2.5, G é nilpotente.

Se $|\zeta(G)|=\infty$, então, como $G$ não tem elementos de ordem 2 , temos que $\left|\zeta(G)^{2}\right|=\infty$. Assim, pela Proposição 3.1.6, obtemos que $F G$ é Lie nilpotente; donde $G^{\prime}$ é um p-grupo finito. Desta forma, pode-se supor $|\zeta(G)|<\infty$. Do Lema 3.2.1, pode-se assumir que $\zeta(G)$ é um p-grupo finito. Aplicando indução sobre a classe de nilpotência de $G$, temos $(G / \zeta(G))^{\prime}$ é um $p$-grupo finito. Logo $G^{\prime}$ é um p-grupo finito. Do Teorema 2.1.2, FG é Lie nilpotente.

O caso em que $(F G)^{-}$é Lie nilpotente é similar.

Novamente, a recíproca é trivial. 


\section{Capítulo 4}

\section{Grupos em geral}

G.T. Lee caracterizou, em [22] e [23], quando o conjunto dos elementos simétricos sob a involução clássica é Lie nilpotente e Lie $n$-Engel. Neste capítulo estudaremos estas duas propriedades em $(F G)^{+}$, sob a involução clássica orientada, discutindo separadamente os casos em que $Q_{8}$ está contido em $G$ ou não.

\subsection{Grupos que contêm uma cópia de $Q_{8}$}

Necessitamos da seguinte definição

Definição 4.1.1. Um grupo $G$ diz-se um produto central interno de dois subgrupos normais $H$ e $K$ se $G=H K,(H, K)=1$ e $H \cap K \subseteq \zeta(G)$. Denotaremos este fato escrevendo $G=H \times_{\zeta} K$.

Lema 4.1.2. Sejam $G$ um grupo e $A$ um subgrupo de indice 2 em $G$. Suponha $A=C \times E$, um produto direto de grupos, com E um 2-grupo abeliano elementar. Se E é central em G, então, para qualquer $g \in G \backslash A, G$ é um produto central dos subgrupos $\langle C, g\rangle$ e E.

Demonstração. Seja $g \in G \backslash A$. Como $A$ é de índice $2 \mathrm{em} G, E$ é central em $G$ e $A=C \times E$, temos $G=\langle C, g\rangle E$. Claramente $\langle C, g\rangle \cap E \subseteq \zeta(G)$.

Lema 4.1.3. Sejam F um corpo de característica $p>2, G$ um grupo com uma orientação não trivial $\sigma$ e $x, y$ elementos de $G$ tais que $\langle x, y\rangle \simeq Q_{8} . S e(F G)^{+}$é Lie $p^{m}$-Engel para algum $m>0$, então existe $g \in G \backslash N$ tal que $g^{2 p^{m}} \neq 1$ e $g^{2}=x^{2}$. Além disso, $(h, x)=(h, y)=1$ para todo $h \in G \backslash N$ tal que $h^{2 p^{m}} \neq 1$. 
Demonstração. Suponha, por absurdo, $a^{2 p^{m}}=1$, para todo $a \in G \backslash N$. Seja $g \in G \backslash N$. Assuma primeiro $o(g) \neq 2$. Como $(F G)^{+}$é Lie $p^{m}$-Engel e $o(x)=4$, temos $\left[g-g^{-1}, x^{p^{m}}+\right.$ $\left.x^{-p^{m}}\right]=\left[g-g^{-1}, x+x^{-1}\right]=0$. Usando a parte (iii) do Lema 3.1.2, temos $g x=x g$, $g x=x^{-1} g$ ou $o(g)=4$. A última possibilidade é descartada pois $g^{2 p^{m}}=1$. Se $g x=x g$, então $(g x)^{2 p^{m}}=g^{2 p^{m}} x^{2 p^{m}}=1$ e assim $x^{2 p^{m}}=1$, uma contradição. Desta forma devemos ter $g x=x^{-1} g$.

Agora suponha $o(g)=2$. Assuma que $(g x)^{2} \neq 1$. Pelo argumento anterior $(g x) x=$ $x^{-1}(g x)$ e assim $g x=x^{-1} g$ o que implica $(g x)^{2}=1$. Desta forma, $(g x)^{2}=1$. Então $g x=$ $x^{-1} g^{-1}=x^{-1} g$. Assim, $g x=x^{-1} g$. Logo $g x=x^{-1} g$, para todo $g \in G \backslash N$. Como $g y \in G \backslash N$, devemos ter $(g y) x=x^{-1}(g y)$, o que implica $x^{2}=1$, uma contradição.

Portanto, existe um $g \in G \backslash N$ tal que $g^{2 p^{m}} \neq 1$. Seja $h \in G \backslash N$ tal que $h^{2 p^{m}} \neq 1$. Dado que $(F G)^{+}$é Lie $p^{m}$-Engel, temos $\left[h-h^{-1}, x+x^{-1}\right]=0$, pois $o(x)=4$. Aplicando a parte (iii) do Lema 3.1.2 obtemos:

(1) $h x=x h$ ou

(2) $h x=x^{-1} h$ ou

(3) $x^{2}=h^{2}$ e $o(h)=4$.

Primeiro mostraremos que $h x=x^{-1} h$ leva a uma contradição. De fato, se $h x=x^{-1} h$, é fácil verificar as identidades:

$$
(h x)^{k}= \begin{cases}h^{k}, & \text { se } k \text { é par } \\ h^{k} x, & \text { se } k \text { é ímpar. }\end{cases}
$$

Assim, $(h x)^{2 p^{m}}=h^{2 p^{m}} \neq 1$. Considerando $\left[h-h^{-1},(h x)^{p^{m}}-(h x)^{-p^{m}}\right]=0$, do Corolário 3.1 .3 , temos $h(h x)^{p^{m}}=(h x)^{p^{m}} h$ e assim $h h^{p^{m}} x=h^{p^{m}} x h$, donde $h x=x h$ e isto com a hipótese inicial implica $x^{2}=1$, uma contradição.

Como acima, podemos provar a igualdade $\left[h-h^{-1}, y+y^{-1}\right]=0$, que implica

(i) $h y=y h$ ou

(ii) $h y=y^{-1} h$ ou 
(iii) $y^{2}=h^{2}$ e $o(h)=4$.

e, de novo, segue $h y=y^{-1} h$, que é uma contradição.

Como nossas condições são simétricas em $x$ e $y$, devemos estudar unicamente três possibilidades: (1)-(i), (1)-(iii) e (3)-(iii).

Suponha que (1) e (iii) sejam válidas. Isto significa $h x=x h, o(h)=4$ e $h^{2}=y^{2}$. Se $(y h)^{2}=1$, então $y h=h^{-1} y^{-1}=h^{3} y^{-1}=h y^{2} y^{-1}=h y$. Suponha $(y h)^{2} \neq 1$. Como $o(h)=4$ e $\left[(y h)-(y h)^{-1}, h^{p^{m}}-h^{-p^{m}}\right]=0$, obtemos $\left[(y h)-(y h)^{-1}, h-h^{-1}\right]=0$. Do Corolário 3.1.3 sabemos que $(y h, h)=1$ e obtemos $(y, h)=1$.

Se (3) e (iii) são satisfeitas, então $o(h)=4$ e $x^{2}=y^{2}=h^{2}$. Se $(x h)^{2} \neq 1$, então $\left[x h-(x h)^{-1}, h-h^{-1}\right]=0$, pelo Corolário 3.1.3 $(x h, h)=1$, e assim $(x, h)=1$. Se $(x h)^{2}=1$, então $x h=h^{-1} x^{-1}=h^{3} x^{-1}=h x^{2} x^{-1}=h x$. Logo $x h=h x$. Similarmente, podemos mostrar que $y h=h y$.

Portanto, em todos os casos possíveis, temos $(h, x)=(h, y)=1$.

Finalmente, suponha $(x h)^{2} \neq 1$ e $(y h)^{2 p^{m}} \neq 1$, para todos os elementos $h \in G \backslash N$, como acima. Então $\left[x h-(x h)^{-1},(y h)^{p^{m}}-(y h)^{-p^{m}}\right]=0$. Logo, do Corolário 3.1.3, $x h y^{p^{m}} h^{p^{m}}=$ $y^{p^{m}} h^{p^{m}} x h$ e assim $x y=y x$, uma contradição. Portanto, existe $g \in G \backslash N$ tal que $g^{2 p^{m}} \neq 1 \mathrm{e}$ $(x g)^{2}=1$ ou $(y g)^{2 p^{m}}=1$.

Se $(x g)^{2}=1$, então $x^{2} g^{2}=1$, o que implica $x^{2}=g^{2}$. Similarmente, $(y g)^{2 p^{m}}=1$ implica $y^{2}=g^{2 p^{m}}$. Neste caso, tomamos $g_{1}=g^{p^{m}}$.

Em qualquer caso, encontramos um elemento $g \in G \backslash N$ com as características do enunciado.

Lema 4.1.4. Sejam $R$ um anel comutativo, $Q_{8}=\langle x, y\rangle$ o grupo quatérnio de ordem 8 e $G=\left\langle Q_{8}, g\right\rangle, \operatorname{com}(g, x)=(g, y)=1$ e $g^{2}=x^{2}$. Seja $\sigma$ a orientação de $G$ definida por $\sigma(x)=\sigma(y)=1$ e $\sigma(g)=-1$. Então $(R G)^{+}$é central em $R G$.

Demonstração. Lembramos que $(R G)^{+}$é gerado como um $R$-módulo pelo conjunto

$$
\mathcal{S}=\left\{1, x^{2}\right\} \cup\left\{x+x^{-1}, y+y^{-1}, x y+(x y)^{-1}\right\} \cup\left\{g-g^{-1}\right\}
$$

Para qualquer $\gamma \in \mathcal{S}$, é fácil verificar as igualdades $[\gamma, x]=[\gamma, y]=[\gamma, g]=0$, assim $(R G)^{+}$ é central em $R G$. 
Teorema 4.1.5. Sejam F um corpo de característica $p \neq 2, G$ um grupo com uma orientação não trivial $\sigma$ e $x, y$ elementos de $G$ tais que $\langle x, y\rangle \simeq Q_{8}$. Então $(F G)^{+}$é Lie $n$-Engel, para algum $n \in \mathbb{N}$ se, e somente se, vale uma das seguintes condições:

(i) $\operatorname{car}(F)=0, N \simeq Q_{8} \times E$ e $G \simeq\left\langle Q_{8}, g\right\rangle \times E$, onde $E^{2}=1$, e $g \in G \backslash N$ é tal que $(g, x)=(g, y)=1$ e $g^{2}=x^{2}$ ou

(ii) $\operatorname{car}(F)=p>2, N \simeq Q_{8} \times E \times P$, onde $P$ é um p-grupo nilpotente de expoente limitado que contém um subgrupo normal p-abeliano $A$ de indice finito e existe $g \in G \backslash N$ tal que $G \simeq\left\langle Q_{8}, g\right\rangle \times E \times P,(g, x)=(g, y)=1$ e $g^{2}=x^{2}$.

Demonstração. Suponha $\operatorname{car}(F)=0$. Assuma que $(F G)^{+}$seja Lie $n$-Engel. Do Lema 3.1.4 temos $Q_{8} \subseteq N$ e, do Teorema 2.1.7, $N \simeq Q_{8} \times E$, onde $E$ é um 2-grupo abeliano elementar. Dado que $F G$ é semiprimo e $N$ não é abeliano, dos Teoremas 2.1.8 e 2.1.9, temos $G \simeq\left\langle x, y, g: x^{4}=1, y^{2}=x^{2}=g^{2}, x^{y}=x^{-1}, x^{g}=x, y^{g}=y\right\rangle \times E$. Reciprocamente, temos $(F G)^{+}=\left(F\left(\left\langle Q_{8}, g\right\rangle \times E\right)\right)^{+}=\left((F(E))\left\langle Q_{8}, g\right\rangle\right)^{+}$e, do Lema 4.1.4, os elementos simétricos comutam.

Consideremos agora o caso em que $\operatorname{car}(F)=p>2$. Suponha que $(F G)^{+}$seja Lie $n$-Engel, para algum $n$. Do Lema 3.1.4, temos $Q_{8} \subseteq N$ e, do Teorema 2.1.7, temos $N \simeq Q_{8} \times E \times P$, onde $E^{2}=1$ e $P$ é um $p$-grupo nilpotente de expoente limitado que contém um subgrupo normal $p$-abeliano $A$ de índice finito. Do Lema 3.1.1, $E$ é central em $G$ e assim o Lema 4.1.2 implica $G \simeq\left\langle Q_{8} \times P, g\right\rangle \times_{\zeta} E$, onde $g$ é qualquer elemento de $G \backslash N$. Dado que $(F G)^{+}$é Lie $n$-Engel, existe um inteiro $m>0$ tal que $p^{m} \geq n$ e

$$
[\gamma, \underbrace{\beta, \ldots, \beta}_{p^{m} \text { vezes }}]=\left[\gamma, \beta^{p^{m}}\right]=0 \text {, para todo } \gamma, \beta \in(F G)^{+} .
$$

Do Lema 4.1.3, qualquer elemento $h \in G \backslash N$ tal que $h^{2 p^{m}} \neq 1$ comuta com $Q_{8}$. Também o Lema 4.1.3 garante a existência de pelo menos um elemento $g \in G \backslash N$ tal que $g^{2 p^{m}} \neq 1$, $g^{2}=x^{2},(g, x)=(g, y)=1$. Portanto, $o(g)=4$.

Seja $t$ um elemento arbitrário de $P$. Temos $\left[t+t^{-1}, g^{p^{m}}-g^{-p^{m}}\right]=0$, donde $\left[t+t^{-1}, g-g^{-1}\right]=0$. Da parte (iii) do Lema 3.1.2, temos $g t=t g, g t=t^{-1} g$ ou $o(t)=4$. Como $t$ é um $p$-elemento, a última opção não pode acontecer. Se $g t=t^{-1} g$, então $(g t)^{2}=g^{2} \neq 1$. 
Como $\left[g t-(g t)^{-1}, g-g^{-1}\right]=0$, do Corolário 3.1.3, tem-se $g t=t g$ e assim $t^{2}=1$, donde $t=1$. Assim, temos $(g, t)=1$. Pelo argumento anterior, $(g, P)=1$.

Pelo Lema 4.1.2, temos $G \simeq\left\langle Q_{8} \times P, g\right\rangle \times_{\zeta} E$ e pelas observações anteriores, temos $\left\langle Q_{8} \times P, g\right\rangle=\left\langle Q_{8}, g\right\rangle \times P$. Na verdade, neste caso, o produto central é um produto direto. Com efeito, tome $\ell \in\left(\left\langle Q_{8}, g\right\rangle \times P\right) \cap E$. Como $E \subseteq N$, temos que $g$ aparece um número par de vezes na expressão de $\ell$ como um elemento do grupo $\left\langle Q_{8}, g\right\rangle \times P$ e, como $g$ comuta com os elementos de $Q_{8}$ e $P$, podemos escrever $\ell=z t g^{2 r}$, para alguns $r \in \mathbb{Z}, z \in Q_{8}$ e $t \in P$. Lembrando que $g^{2}=x^{2}$, temos $\ell \in\left(Q_{8} \times P\right) \cap E=1$. Portanto, $G \simeq\left\langle Q_{8}, g\right\rangle \times E \times P$.

Reciprocamente, seja $g \in G \backslash N$ tal que $G=\left\langle Q_{8}, g\right\rangle \times E \times P,(g, x)=(g, y)=1$ e $g^{2}=x^{2}$. Como $g$ é central em $G$, temos $\left[g-g^{-1}, \gamma\right]=0$, para todo $\gamma \in(F G)^{+}$. Queremos provar que existe $s>0$ tal que $\left[\gamma, \beta^{p^{s}}\right]=0$, para todo $\gamma, \beta \in(F G)^{+}$. Primeiro, note que podemos escrever $\beta=\beta_{1}+\beta_{2}$, onde $\beta_{1}=\sum_{h \in N} \beta_{h} h$ e $\beta_{2}=\sum_{h \in(G \backslash N)} \beta_{h} h$.

Assim $\beta_{1} \in(F N)^{+}$e podemos escrevê-lo como uma combinação linear de elementos da forma $a_{1} c_{1}+a_{1}^{-1} c_{1}^{-1}$, com $a_{1} \in Q_{8} \times E$ e $c_{1} \in P$. Além disso, $a_{1} c_{1}+a_{1}^{-1} c_{1}^{-1}=$ $a_{1}+a_{1}^{-1}+a_{1}\left(c_{1}-1\right)+a_{1}^{-1}\left(c_{1}^{-1}-1\right)$. Do Lema 4.1.4, $a_{1}+a_{1}^{-1}$ é central em $F\left(\left\langle Q_{8}, g\right\rangle \times E\right)$ e também em $F G$. Logo podemos expressar $\beta_{1}$ na forma $\alpha_{1}+\delta_{1}$, onde $\alpha_{1}$ é central em $F G$ e $\delta_{1} \in \Delta(G, P)$.

Por outro lado, escrevemos $\beta_{2}$ como combinação linear de elementos da forma $g a_{2} c_{2}-$ $g^{-1} a_{2}^{-1} c_{2}^{-1}, \quad$ com $a_{2} \in Q_{8} \times E$ e $c_{2} \in P$. Temos $g a_{2} c_{2}-g^{-1} a_{2}^{-1} c_{2}^{-1}=$ $g a_{2}-\left(g a_{2}\right)^{-1}+g a_{2}\left(c_{2}-1\right)-g^{-1} a_{2}^{-1}\left(c_{2}^{-1}-1\right)$ e, pelo Lema 4.1.4, $g a_{2}-\left(g a_{2}\right)^{-1}$ é central em $F\left(\left\langle Q_{8}, g\right\rangle \times E\right)$. Logo podemos escrever $\beta_{2}$ como $\alpha_{2}+\delta_{2}$, onde $\alpha_{2}$ é central em $F G$ e $\delta_{2} \in \Delta(G, P)$.

Das duas últimas observações temos $\beta=\alpha+\delta$, com $\alpha$ um elemento central em $F G$ e $\delta \in \Delta(G, P)$. Dado que

$$
\frac{G}{E \times A}=\frac{\left\langle Q_{8}, g\right\rangle \times E \times P}{E \times A} \simeq\left\langle Q_{8}, g\right\rangle \times P / A,
$$

$E \times A$ é um subgrupo p-abeliano de índice finito em G. Assim, da Proposição 1.1.4 em [24], $F G$ satisfaz uma identidade polinomial. Logo, de [24, Lema 1.3.14], obtemos que $\Delta(G, P)$ é nil de expoente $p^{r}$, para algum $r \in \mathbb{N}$. Daí, $\beta^{p^{r}}=(\alpha+\delta)^{p^{r}}=\alpha^{p^{r}}+\delta^{p^{r}}=\alpha^{p^{r}}$. Consequente- 
mente, temos $\left[\gamma, \beta^{p^{r}}\right]=0$, para todo $\gamma, \beta \in(F G)^{+}$. Portanto, provamos que $(F G)^{+}$é Lie $p^{r}$-Engel.

Teorema 4.1.6. Sejam F um corpo de característica $p \neq 2$, G um grupo com uma orientação não trivial $\sigma$ e $x, y$ elementos de $G$ tais que $\langle x, y\rangle \simeq Q_{8}$. Então $(F G)^{+}$é Lie nilpotente se, e somente se, vale uma das seguintes condiçôes

(i) $\operatorname{car}(F)=0, N \simeq Q_{8} \times E$ e $G \simeq\left\langle Q_{8}, g\right\rangle \times E$, onde $E^{2}=1$ e $g \in G \backslash N$ é tal que $(g, x)=(g, y)=1$ e $g^{2}=x^{2}$ ou

(ii) $\operatorname{car}(F)=p>2, N \simeq Q_{8} \times E \times P$, onde $E^{2}=1, P$ é um p-grupo finito e existe $g \in G \backslash N$ tal que $G \simeq\left\langle Q_{8}, g\right\rangle \times E \times P,(g, x)=(g, y)=1$ e $g^{2}=x^{2}$.

Demonstração. Assuma $\operatorname{car}(F)=0$ e $(F G)^{+}$é Lie nilpotente, então, do Teorema 4.1.5, o resultado é imediato. Reciprocamente, do Lema 4.1.4, $(F G)^{+}$é comutativo.

Agora, suponha $\operatorname{car}(F)=p>2$ e que $(F G)^{+}$é Lie nilpotente. Do Lema 3.1.4 e do Teorema 2.1.5, temos $N \simeq Q_{8} \times E \times P$, onde $E^{2}=1$ e $P$ é um $p$-grupo finito. Assim, do Teorema 4.1.5, existe $g \in G \backslash N$ tal que $G \simeq\left\langle Q_{8}, g\right\rangle \times E \times P,(g, x)=(g, y)=1$ e $g^{2}=x^{2}$.

Reciprocamente, suponha $|P|=p^{n}$. Afirmamos que, para quaisquer $p^{n}+1$ elementos simétricos $\gamma_{1}, \ldots, \gamma_{p^{n}+1},\left[\gamma_{1}, \ldots, \gamma_{p^{n}+1}\right]=0$. A prova será por indução sobre $n$. Se $n=0$, então $G \simeq\left\langle Q_{8}, g\right\rangle \times E$ e assim o Lema 4.1 .4 implica $(F G)^{+}$comutativo.

Suponha $n \geq 1$. Tome $z \in \zeta(P)$ tal que $o(z)=p$. Como $\sigma(z)=1$, podemos considerar a orientação induzida $\bar{\sigma}$ definidas sobre $G /\left\langle z^{2}\right\rangle$. Assim; pela hipótese indutiva, trabalhando sobre $F\left(G /\left\langle z^{2}\right\rangle\right)$, obtemos $\left[\bar{\gamma}_{1}, \ldots, \bar{\gamma}_{p^{n-1}+1}\right]=0$, para todo $\bar{\gamma}_{i} \in\left(F\left(G /\left\langle z^{2}\right\rangle\right)\right)^{+}$. Assim $\left[\gamma_{1}, \ldots, \gamma_{p^{n-1}+1}\right] \in \Delta\left(G,\left\langle z^{2}\right\rangle\right)=\left(z^{2}-1\right) F G=\left(z-z^{-1}\right) F G . \operatorname{Logo}\left[\gamma_{1}, \ldots, \gamma_{p^{n-1}+1}\right]=(z-$ $\left.z^{-1}\right) w$, para algum $w \in F G$.

É fácil verificar as inclusões $\left[(F G)^{+},(F G)^{+}\right] \subseteq(F G)^{-}$e $\left[(F G)^{-},(F G)^{+}\right] \subseteq(F G)^{+}$. Como $p^{n-1}+1$ é par, $\left(z-z^{-1}\right) w=\left[\gamma_{1}, \ldots, \gamma_{p^{n-1}+1}\right] \in(F G)^{-}$. Assim, $\left(\left(z-z^{-1}\right) w\right)^{*}=-\left(z-z^{-1}\right) w$. Como $z-z^{-1}$ é central e anti-simétrico, também temos $\left(\left(z-z^{-1}\right) w\right)^{*}=-\left(z-z^{-1}\right) w^{*}$. Assim, $\left(z-z^{-1}\right) w=\left(z-z^{-1}\right) w^{*}$, donde

$$
\left(z-z^{-1}\right) w=\left(z-z^{-1}\right) \beta_{1} \text {, onde } \beta_{1}=\frac{w+w^{*}}{2} \text { é simétrico. }
$$


Assim, $\left[\gamma_{1}, \ldots, \gamma_{2 p^{n-1}+1}\right]=\left(z-z^{-1}\right)\left[\beta_{1}, \gamma_{p^{n-1}+2}, \ldots, \gamma_{2 p^{n-1}+1}\right]$. Como acabamos de observar, $\left[\beta_{1}, \gamma_{p^{n-1}+2}, \ldots, \gamma_{2 p^{n-1}+1}\right]=\left(z-z^{-1}\right) \beta_{2}$, para algum $\beta_{2} \in(F G)^{+}$(pois é o colchete de Lie de $p^{n-1}+1$ elementos simétricos). Iterando este argumento, obtemos $\left[\gamma_{1}, \ldots, \gamma_{p^{n}+1}\right]=$ $\left(z-z^{-1}\right)^{p} \beta_{p}$, para algum $\beta_{p} \in(F G)^{+}$. Como $\left(z-z^{-1}\right)^{p}=0$, isto completa a prova.

Agora abordamos o caso em que $(F G)^{+}$é fortemente Lie nilpotente. Lembremos o Lema 3.3.3 de [24].

Lema 4.1.7. Seja $R$ um anel e $S$ um subconjunto de $R$. Suponha, para algum $i \geq 1$, $S^{(i)} \subseteq z R$, onde z é central em $R$. Então, para todo $j>0$, temos $S^{(i+j)} \subseteq z S^{(j)}$. Em particular, para qualquer inteiro positivo $m, S^{(m i)} \subseteq z^{m} R$.

Demonstração. A prova é por indução sobre $j$. Se $j=1$, como $S^{(i+1)} \subseteq S^{(i)}$, não temos nada a provar. Suponha $S^{(i+j)} \subseteq z S^{(j)}$. Tomemos $a \in S^{(i+j)}, b \in S$. Então $a=z a_{1}$, para algum $a_{1} \in S^{(j)}$. Assim, $[a, b]=\left[z a_{1}, b\right]=z\left[a_{1}, b\right] \in z S^{(i+j)}$, como queríamos provar.

Para obter a segunda parte, considerando

$$
S^{(2 i)}=S^{(i+i)} \subseteq z S^{(i)} \subseteq z^{2} R
$$

e supondo $S^{((m-1) i)} \subseteq z^{m-1} R$, temos $S^{(m i)}=S^{((m-1) i+i)} \subseteq z S^{((m-1) i)} \subseteq z^{m} R$.

Teorema 4.1.8. Sejam F um corpo de característica $p \neq 2$, G um grupo com uma orientação não trivial $\sigma$ e $x, y$ elementos de $G$ tais que $\langle x, y\rangle \simeq Q_{8}$. Então $(F G)^{+}$é fortemente Lie nilpotente se, e somente se, vale uma das seguintes condições

(i) $\operatorname{car}(F)=0, N \simeq Q_{8} \times E$ e $G \simeq\left\langle Q_{8}, g\right\rangle \times E$, onde $E^{2}=1$ e $g \in G \backslash N$ é tal que $(g, x)=(g, y)=1$ e $g^{2}=x^{2}$ ou

(ii) $\operatorname{car}(F)=p>2, N \simeq Q_{8} \times E \times P$, onde $E^{2}=1, P$ é um p-grupo finito e existe $g \in G \backslash N$ tal que $G \simeq\left\langle Q_{8}, g\right\rangle \times E \times P,(g, x)=(g, y)=1$ e $g^{2}=x^{2}$.

Demonstração. Se $(F G)^{+}$é fortemente Lie nilpotente, então $(F G)^{+}$é Lie nilpotente e do Teorema 4.1.6 obtemos (i) e (ii).

Reciprocamente, suponha $|P|=p^{n}$. Afirmamos que $\left((F G)^{+}\right)^{2 p^{n}}=0$. A prova será por indução sobre $n$. Se $n=0$, então $G \simeq\left\langle Q_{8}, g\right\rangle \times E$ e assim $(F G)^{+}$é comutativo. Assuma 
$|P|=p^{n}>1$. Tomando $z \in \zeta(P)$ com $o(z)=p$, aplicamos nossa hipótese de indução para $\bar{G}=G /\langle z\rangle$. Então $\left((F \bar{G})^{+}\right)^{\left(2 p^{n-1}\right)}=0$. Assim

$$
\left((F G)^{+}\right)^{\left(2 p^{n-1}\right)} \subseteq \Delta(G,\langle z\rangle)=(z-1) F G
$$

Pelo Lema 4.1.7,

$$
\left((F G)^{+}\right)^{\left(2 p^{n}\right)} \subseteq(z-1)^{p} F G=0,
$$

como queríamos mostrar.

Pelo Lema 2.2.4, obtemos o seguinte corolário.

Corolário 4.1.9. Sejam $F$ um corpo de característica diferente de 2 . Assuma $Q_{8} \subseteq G$ e que $(F G)^{+}$é Lie nilpotente. Então $\mathcal{U}^{+}(F G)$ é nilpotente.

\subsection{Outros resultados}

Nesta seção apresentamos resultados no caso em que $G$ não contém subgrupo isomorfo a $Q_{8}$ e a álgebra de grupo $F G$ é semiprima. Em [24, p. 86], G.T. Lee observou que ao estudar as propriedades de Lie dos elementos anti-simétricos sob a involução clássica os resultados obtidos em grupos sem elementos de ordem 2 não podem ser estendidos a grupos que não contêm os quatérnios. Dificuldades similares são encontradas no contexto atual.

Seja $D_{k}=\langle x, y\rangle$ um grupo diedral. É fácil ver que a única orientação $\sigma$ tal que $\left(F D_{k}\right)^{+}$ é Lie nilpotente é dada por $\sigma(x)=1$ e $\sigma(y)=-1$. De fato, se $\left(F D_{k}\right)^{+}$é Lie nilpotente e $\sigma(y)=1$ então, do Lema 3.1.1, y é um elemento central, uma contradição. Logo $\sigma(y)=-1$. Também, se $\sigma(x)=-1$ então , pelo Lema 3.1.1, xy é central, outra contradição. Portanto, $\sigma(x)=1$.

Neste caso $N=\operatorname{ker}(\sigma)=\langle x\rangle, G \backslash N=\left\{x^{i} y: 0 \leq i \leq k-1\right\},(G \backslash N)^{2}=1$ e assim $\left(F D_{k}\right)^{+}$é comutativo, mas $F D_{k}$ não é Lie nilpotente quando car $(F) \neq 2$.

Lema 4.2.1. Seja $G$ um grupo tal que $Q_{8} \not \subset G$ com uma orientação não trivial $\sigma$ e $\operatorname{car}(F) \neq$ 2. Assuma $g^{2} \neq 1$, para todo $g \in G \backslash N$ e, que $(F G)^{+}$é Lie $n$-Engel, para algum $n$. Então:

(i) se $\operatorname{car}(F)=0$, então $G$ é abeliano; 
(ii) se $\operatorname{car}(F)=p>0$, então $G^{p^{m}} \subseteq \zeta(G)$, para algum $m>0$.

Demonstração. Suponha $\operatorname{car}(F)=0$. Então $F G$ é semiprimo e, do Teorema 2.1.8, temos $\left[(F G)^{+},(F G)^{+}\right]=0$. Então o Teorema 2.1.9 implica $G$ abeliano.

Suponha $\operatorname{car}(F)=p>2$. Escolha $m>0$ tal que $p^{m} \geq n$. Seja $b \in G$ um elemento fixo e $a \in G$ um elemento qualquer. Consideraremos os seguintes casos:

1. $\sigma(a)=\sigma(b)=1$.

De [22, Lema 5], obtemos $\left(a, b^{p^{m}}\right)=1$.

2. $\sigma(a)=\sigma(b)=-1$.

Pela hipótese, $a^{2} \neq 1$ e $b^{2 p^{m}} \neq 1$. Do Corolário 3.1.3, obtemos $\left(a, b^{p^{m}}\right)=1$.

3. $\sigma(a)=1$ e $\sigma(b)=-1$.

Tome $c=a b$. Pelo item anterior obtemos $\left(c, b^{p^{m}}\right)=1$, o que implica $\left(a, b^{p^{m}}\right)=1$.

4. $\sigma(a)=-1$ e $\sigma(b)=1$.

Do primeiro item $\left(a^{2}, b^{p^{m}}\right)=1$. Agora, do item anterior, temos $\left(b, a^{p^{m}}\right)=1$. Como $2 \mathrm{e}$ $p$ são relativamente primos, resulta $\left(a, b^{p^{m}}\right)=1$.

Teorema 4.2.2. Seja $F$ um corpo de característica $p \neq 2$ e $G$ um grupo tal que $Q_{8} \nsubseteq G$ com orientação não trivial $\sigma$. Suponha $g^{2} \neq 1$, para todo $g \in G \backslash N$. Então $(F G)^{+}$é Lie n-Engel, para algum n, se, e somente se, FG é Lie $m$-Engel, para algum $m$.

A prova é similar à prova do Teorema 3.2.5 usando o Lemma 4.2.1 no lugar do Lema 3.2.4.

Teorema 4.2.3. Seja $F$ um corpo de característica $p \neq 2$ e $G$ um grupo tal que $Q_{8} \nsubseteq G$ com uma orientação não trivial $\sigma$. Suponha $g^{2} \neq 1$, para todo $g \in G \backslash N$. Então $(F G)^{+}$é Lie nilpotente se, e somente se, FG é Lie nilpotente.

Demonstração. Assuma $(F G)^{+}$Lie nilpotente. Se $\operatorname{car}(F)=0$, então o resultado já foi provado no Lema 4.2.1. 
Seja então $\operatorname{car}(F)=p>2$. Do Teorema 4.2.2, $G$ é nilpotente.

Como $G$ é nilpotente e sabemos, do Lema 4.2.1, que $G / \zeta(G)$ é um p-grupo de expoente limitado, de [24, Proposição 1.3.7], $G^{\prime}$ é um p-grupo. Suponha que $G$ contém um elemento $x$ de ordem infinita. Pelo Lema 4.2.1 alguma potência positiva de $x$ é central, e assim $\left|\zeta(G)^{2}\right|=\infty$. Pela Proposição 3.1.6, obtemos que FG é Lie nilpotente. Portanto, podemos assumir que $G$ é de torção. Então $G$ é um produto direto $\prod_{q} P_{q}$ onde, para cada primo $q$, $P_{q}$ é o único $q$-subgrupo de Sylow de $G$ (vide [31, Teorema 5.2.7]). Como $G^{\prime}$ é um $p$-group, $G^{\prime}=P_{p}^{\prime}$. Dado que $\left(F P_{p}\right)^{+}$é Lie nilpotente, do Teorema 2.1.4, $P_{p}^{\prime}$ é finito. Finalmente, pelo Teorema 2.1.2 resulta que $F G$ é Lie nilpotente.

A recíproca é trivial.

Proposição 4.2.4. Sejam F um corpo de característica diferente de 2 e $G$ um grupo tal que $F G$ é semiprimo e $Q_{8} \nsubseteq G$. Então $(F G)^{+}$é Lie $n$-Engel, para algum $n$ se, e somente se, uma das seguintes condições é verificada

(i) G é abeliano ou

(ii) $N=\operatorname{Ker}(\sigma)$ é abeliano e $(G \backslash N)^{2}=1$.

Demonstração. É claro que qualquer uma das opções acima implica $(F G)^{+}$Lie $n$-Engel. Suponha que $(F G)^{+}$é Lie $n$-Engel. Como $Q_{8} \not \subseteq G$ e $\operatorname{car}(F) \neq 2$, dos Teoremas 2.1.8 e 2.1.9 obtemos o resultado. 


\section{Capítulo 5}

\section{Índices de Lie Nilpotência}

Neste capítulo estudamos os índices de Lie nilpotência do conjunto de elementos simétricos sob uma involução orientada.

\subsection{Preliminares}

Como observamos no Teorema 2.1.2, numa álgebra de grupo $F G$, os conceitos de Lie nilpotência e fortemente Lie nilpotência são equivalentes.

De [33], se $F G$ é Lie nilpotente, então $\mathrm{t}(F G) \leq \mathrm{t}^{\mathrm{L}}(F G) \leq\left|G^{\prime}\right|+1$ e, por [6], a igualdade é satisfeita quando $G^{\prime}$ é cíclico ou quando $G^{\prime}$ é um grupo abeliano elementar, não central, de ordem 4.

Como antes, com * denotaremos uma involução clássica orientada e com $N$ o kernel da orientação não trivial $\sigma$. Vamos estudar o índice de Lie nilpotência de $(F G)^{+}$sob uma involução clássica orientada. Os resultados apresentados neste capítulo são baseados nos artigos [5] e [4] que estendemos à situação presente. Mostremos primeiro o seguinte resultado.

Lema 5.1.1. Sejam $H$ um subgrupo de um grupo $G$ e $A$ um subgrupo normal de $G$ tal que $A \subseteq N . S e(F G)^{+}$é Lie nilpotente, então $(F H)^{+} e(F(G / A))^{+}$também são Lie nilpotentes. Além disso, $\mathrm{t}\left((F H)^{+}\right) \leq \mathrm{t}\left((F G)^{+}\right)$e $\mathrm{t}\left((F(G / A))^{+}\right) \leq \mathrm{t}\left((F G)^{+}\right)$.

Demonstração. Sabemos que $(F H)^{+}$é um subconjunto de $(F G)^{+}$e isto leva aos resultados desejados. Dado que $A$ é um subgrupo normal contido no núcleo da orientação $\sigma$, podemos 
definir em $F(G / A)$ uma involução orientada a partir da involução * de $F G$ como segue:

$$
\left(\sum_{\bar{g} \in G / A} \alpha_{g} \bar{g}\right)^{\star}=\sum_{\bar{g} \in G / A} \alpha_{g} \sigma(g) \bar{g}^{-1}
$$

Simplesmente observamos que os elementos simétricos em $F(G / A)$ sob « são combinações lineares de termos da forma $g A+\sigma(g) g^{-1} A$, com $g \in G$, isto é, todo elemento de $(F(G / A))^{+}$é a imagem homomorfa de um elemento de $(F G)^{+}$sob a aplicação natural $\varepsilon_{A}: F G \rightarrow F(G / A)$.

Assim suponha que $(F G)^{+}$é Lie nilpotente, isto é, que existe $n=\mathrm{t}\left((F G)^{+}\right)$tal que $\left[\alpha_{1}, \ldots, \alpha_{n}\right]=0$, para todo $\alpha_{i} \in(F G)^{+}$. Sejam $\beta_{1}, \ldots, \beta_{n} \in(F(G / A))^{+}$. Logo

$$
\left[\beta_{1}, \ldots, \beta_{n}\right]=\left[\varepsilon_{A}\left(\alpha_{1}\right), \ldots, \varepsilon_{A}\left(\alpha_{n}\right)\right]=\varepsilon_{A}\left(\left[\alpha_{1}, \ldots, \alpha_{n}\right]\right)=\varepsilon_{A}(0)=0
$$

Desta forma, $\mathrm{t}\left((F(G / A))^{+}\right) \leq \mathrm{t}\left((F G)^{+}\right)$.

Suponha primeiro que $F G$ é Lie nilpotente. Claramente, $(F G)^{+}$é Lie nilpotente também, mas não sabemos se $\mathrm{t}(F G)=\mathrm{t}\left((F G)^{+}\right)$. A cota superior $\left|G^{\prime}\right|+1$ de fato diminui com respeito à $\mathrm{t}\left((F G)^{+}\right)$.

Lembramos que, para quaisquer $a, b, c$ num anel associativo $R$, são satisfeitas as seguintes igualdades

$$
\begin{aligned}
& (a b-1)=(a-1)(b-1)+(a-1)+(b-1) \\
& {[a b, c]=a[b, c]+[a, c] b \text { e }[a, b c]=b[a, c]+[a, b] c} \\
& {[a, b]=b a((a, b)-1) \text { e }(a, b)=1+a^{-1} b^{-1}[a, b] \text { (aqui } a \text { e } b \text { são unidades). }}
\end{aligned}
$$

Primeiro damos alguns lemas técnicos que serão necessários. Por definição $\Delta(F G)^{0}=F G$.

Lema 5.1.2. Seja $G$ um grupo tal que $\gamma_{3}(G) \subseteq\left(G^{\prime}\right)^{p}$. Então $[\alpha, \beta, \gamma] \in F G \Delta\left(G^{\prime}\right)^{2}$, para todo $\alpha, \beta, \gamma \in F G$. 
Demonstração. Sejam $x, y, z \in G$ quaisquer. Pelas igualdades (5.3) e (5.2), temos

$$
\begin{aligned}
{[x, y, z] } & =[[x, y], z]=[y x((x, y)-1), z] \\
& =y x[(x, y)-1, z]+[y x, z]((x, y)-1) \\
& =y x[(x, y), z]+[y x, z]((x, y)-1) \\
& =y x z(x, y)((x, y, z)-1)+z y x((y x, z)-1)((x, y)-1) .
\end{aligned}
$$

Assim $[x, y, z] \in F G \Delta\left(\gamma_{3}(G)\right)+F G \Delta\left(G^{\prime}\right)^{2}$. Por hipótese, $\gamma_{3}(G) \subseteq\left(G^{\prime}\right)^{p}$, logo $\Delta\left(\gamma_{3}(G)\right) \subseteq \Delta\left(\left(G^{\prime}\right)^{p}\right) \subseteq \Delta\left(G^{\prime}\right)^{p} \subseteq F G \Delta\left(G^{\prime}\right)^{2}$. Desta forma, $[x, y, z] \in F G \Delta\left(G^{\prime}\right)^{2}$. Pela linearidade do colchete de Lie obtemos o resultado desejado.

O seguinte resultado é o Lema 2 em [2].

Lema 5.1.3 (Lema 2, [2]). Seja $H$ um subgrupo normal de um p-grupo finito G. Então

$$
\Delta(G) \Delta(H)=\Delta(H) \Delta(G)
$$

se, e somente se, $(G, H) \subseteq \Phi(H)$; onde $\Phi(H)$ é o subgrupo de Frattini de $H$.

Lema 5.1.4. Seja $G$ um p-grupo finito tal que $\gamma_{3}(G) \subseteq\left(G^{\prime}\right)^{p}$. Então, para todo $k, l, m, n \geq 1$, tem-se

(i) $\left[\Delta\left(G^{\prime}\right)^{m}, \Delta(G)^{l}\right] \subseteq \Delta(G)^{l-1} \Delta\left(G^{\prime}\right)^{m+1}$;

(ii) $\left[\Delta(G)^{k}, \Delta(G)^{l}\right] \subseteq \Delta(G)^{k+l-2} \Delta\left(G^{\prime}\right)$;

(iii) $\left[\Delta(G)^{k} \Delta\left(G^{\prime}\right)^{m}, \Delta(G)^{l}\right] \subseteq \Delta(G)^{k+l-2} \Delta\left(G^{\prime}\right)^{m+1}$;

(iv) $\left[F G \Delta\left(G^{\prime}\right)^{m}, \Delta(G)^{l}\right] \subseteq F G \Delta\left(G^{\prime}\right)^{m+1}$.

Demonstração. Como $\gamma_{3}(G) \subseteq\left(G^{\prime}\right)^{p} \subseteq \Phi\left(G^{\prime}\right)$, o Lema 5.1 .3 implica $\Delta\left(G^{\prime}\right) \Delta(G)=\Delta(G) \Delta\left(G^{\prime}\right)$.

(i) Do Lema 5.1.2, temos $[\Delta(G), \Delta(G), \Delta(G)] \subseteq F G \Delta\left(G^{\prime}\right)^{2}$. Mostraremos primeiro que $\left[\Delta\left(G^{\prime}\right), \Delta(G)\right] \subseteq F G \Delta\left(G^{\prime}\right)^{2}$. Sejam $z=(x, y) \in G^{\prime}$ e $g \in G, \log \mathrm{O}$

$$
\begin{aligned}
{[z-1, g-1] } & =[z, g]=[z-1, g]=x^{-1} y^{-1}[[x, y], g]+\left[x^{-1} y^{-1}, g\right][x, y] \\
& =x^{-1} y^{-1}[[x, y], g]+g\left(\left(x^{-1} y^{-1}, g\right)-1\right)^{y x}((x, y)-1) \in F G \Delta\left(G^{\prime}\right)^{2}
\end{aligned}
$$


Além disso, para $\alpha=\left(\left(x_{1}, y_{1}\right)-1\right) \cdots\left(\left(x_{s}, y_{s}\right)-1\right)$, com $s \geq 2$, temos

$$
\begin{aligned}
{[\alpha, g-1]=[\alpha, g]=\left(\left(x_{1}, y_{1}\right)-1\right) } & \cdots\left(\left(x_{s-1}, y_{s-1}\right)-1\right)\left[\left(x_{s}, y_{s}\right)-1, g\right]+ \\
& {\left[\left(\left(x_{1}, y_{1}\right)-1\right) \cdots\left(\left(x_{s-1}, y_{s-1}\right)-1\right), g\right]\left(\left(x_{s}, y_{s}\right)-1\right) . }
\end{aligned}
$$

Assim, por indução sobre $m$, obtemos

$$
\begin{aligned}
{\left[\Delta\left(G^{\prime}\right)^{m}, \Delta(G)\right] } & \subseteq \Delta\left(G^{\prime}\right)^{m-1}\left[\Delta\left(G^{\prime}\right), \Delta(G)\right]+\left[\Delta\left(G^{\prime}\right)^{m-1}, \Delta(G)\right] \Delta\left(G^{\prime}\right) \\
& \subseteq F G \Delta\left(G^{\prime}\right)^{m+1} .
\end{aligned}
$$

Desta forma, por indução sobre $l$, segue

$$
\begin{aligned}
{\left[\Delta\left(G^{\prime}\right)^{m}, \Delta(G)^{l}\right] } & \subseteq \Delta(G)^{l-1}\left[\Delta\left(G^{\prime}\right)^{m}, \Delta(G)\right]+\left[\Delta\left(G^{\prime}\right)^{m}, \Delta(G)^{l-1}\right] \Delta(G) \\
& \subseteq \Delta(G)^{l-1} \Delta\left(G^{\prime}\right)^{m+1}
\end{aligned}
$$

(ii) Para $l=1$, temos

$$
[\Delta(G), \Delta(G)] \subseteq F G \Delta\left(G^{\prime}\right)
$$

e, por indução sobre $k$,

$$
\begin{aligned}
{\left[\Delta(G)^{k}, \Delta(G)\right] } & \subseteq \Delta(G)^{k-1}[\Delta(G), \Delta(G)]+\left[\Delta(G)^{k-1}, \Delta(G)\right] \Delta(G) \\
& \subseteq \Delta(G)^{k-1} \Delta\left(G^{\prime}\right) .
\end{aligned}
$$

Logo, por indução sobre $l$,

$$
\begin{aligned}
{\left[\Delta(G)^{k}, \Delta(G)^{l}\right] } & \subseteq \Delta(G)^{l-1}\left[\Delta(G)^{k}, \Delta(G)\right]+\left[\Delta(G)^{k}, \Delta(G)^{l-1}\right] \Delta(G) \\
& \subseteq \Delta(G)^{k+l-2} \Delta\left(G^{\prime}\right)+\Delta(G)^{k+l-3} \Delta\left(G^{\prime}\right) \Delta(G) \\
& \subseteq \Delta(G)^{k+l-2} \Delta\left(G^{\prime}\right) .
\end{aligned}
$$


(iii) O resultado neste item é consequência direta dos items (i) e (ii), já que

$$
\begin{aligned}
{\left[\Delta(G)^{k} \Delta\left(G^{\prime}\right)^{m}, \Delta(G)^{l}\right] } & \subseteq \Delta(G)^{k}\left[\Delta\left(G^{\prime}\right)^{m}, \Delta(G)^{l}\right]+\left[\Delta(G)^{k}, \Delta(G)^{l}\right] \Delta\left(G^{\prime}\right)^{m} \\
& \subseteq \Delta(G)^{k} \Delta(G)^{l-1} \Delta\left(G^{\prime}\right)^{m+1}+\Delta(G)^{k+l-2} \Delta\left(G^{\prime}\right) \Delta\left(G^{\prime}\right)^{m} \\
& \subseteq \Delta(G)^{k+l-1} \Delta\left(G^{\prime}\right)^{m+1}+\Delta(G)^{k+l-2} \Delta\left(G^{\prime}\right)^{m+1} \\
& \subseteq \Delta(G)^{k+l-2} \Delta\left(G^{\prime}\right)^{m+1} .
\end{aligned}
$$

(iv) A última inclusão é consequência dos items (i) e (iii) e da igualdade

$$
F G \Delta\left(G^{\prime}\right)^{m}=\Delta(G) \Delta\left(G^{\prime}\right)^{m}+\Delta\left(G^{\prime}\right)^{m}
$$

Sejam $m$ um inteiro positivo e $g$ um elemento em $G$, então

$$
g^{m}-1=(g-1)\left(g^{m-1}+\cdots+g+1\right)
$$

$\operatorname{assim} g^{m}-m(g-1)=(g-1)\left(\left(g^{m-1}+\cdots+g+1\right)-m\right)$ e, como $g-1, g^{m-1}+\cdots+g+1-m \in$ $\Delta(G)$, obtemos a congruência

$$
g^{m}-1 \equiv m(g-1) \quad\left(\bmod \Delta(G)^{2}\right)
$$

Lema 5.1.5. Seja $G$ um p-grupo finito tal que $G^{\prime}$ é cíclico. Então $\gamma_{3}(G) \subseteq\left(G^{\prime}\right)^{p}$.

Demonstração. Assuma $G^{\prime}=\langle x\rangle$. Como $\gamma_{3}(G) \subseteq G^{\prime}$, temos $\gamma_{3}(G)=\left\langle x^{i}\right\rangle$, para algum inteiro positivo $i$, e, para cada $n>3, \gamma_{n}(G)=\left\langle x^{i k_{n}}\right\rangle$, para algum $k_{n}$. Dado que $G$ é nilpotente, existe $d$ tal que $\gamma_{d}(G)=1$. Assim, $o(x)=p^{r}$ divide $i k_{d}$. Assuma $(i, p)=1$. Isto implica $\gamma_{3}(G)=G^{\prime}$ donde $G^{\prime}=\gamma_{d}(G)=1$ e o resultado segue trivialmente. Por outro lado, se $p$ divide $i$, então $\gamma_{3}(G)=\left\langle x^{p t}\right\rangle=\left\langle\left(x^{t}\right)^{p}\right\rangle$, o que prova o lema.

Lema 5.1.6. Seja $G$ um p-grupo finito com subgrupo comutador $G^{\prime}=\langle x\rangle$ e sejam $a, b \in G$ 
tais que $(a, b)=x$. Então

$$
\left[a^{m}, b^{s}\right] \equiv m s \cdot b^{s} a^{m}(x-1) \quad\left(\bmod F G \Delta\left(F G^{\prime}\right)^{2}\right)
$$

Demonstração. Temos $\left[a^{m}, b^{s}\right]=b^{s} a^{m}\left(\left(a^{m}, b^{s}\right)-1\right)$ e, pelas identidades

$$
\begin{aligned}
& (u, v w)=(u, w)(u, v)(u, v, w) \\
& (u v, w)=(u, w)(u, w, v)(v, w)
\end{aligned}
$$

temos

$$
\left(a^{m}, b^{s}\right) \equiv(a, b)^{m s} \equiv x^{m s} \quad\left(\bmod \gamma_{3}(G)\right)
$$

Pelo Lema 5.1.5, $\gamma_{3}(G) \subseteq\left(G^{\prime}\right)^{p}$, donde $\left(a^{m}, b^{s}\right)=x^{m s+p i}$, para algum $i$. Usando a equação (5.6) e o fato de a característica de $F$ ser $p$, obtemos

$$
\left(a^{m}, b^{s}\right) \equiv(m s+p i)(x-1) \equiv m s \cdot(x-1) \quad\left(\bmod F G \Delta\left(G^{\prime}\right)^{2}\right) .
$$

Para $n \geq 2$, denotamos $I_{n}=\Delta(G)^{3} \Delta\left(G^{\prime}\right)^{n-1}+F G \Delta\left(G^{\prime}\right)^{n}$. Além disso, dados $g, h \in G$ quaisquer, o produto $(g-1)(h-1)(x-1)$ é independente da ordem dos fatores módulo $I_{2}$. Por exemplo, mostramos as congruências:

1. $(g-1)(h-1)(x-1) \equiv(h-1)(g-1)(x-1)\left(\bmod I_{2}\right) \mathrm{e}$

2. $(g-1)(h-1)(x-1) \equiv(g-1)(x-1)(h-1)\left(\bmod I_{2}\right)$.

De fato,

$$
\begin{aligned}
(g-1)(h-1)(x-1)-(h-1)(g-1)(x-1) & =[g, h](x-1) \\
& =h g((g, h)-1)(x-1) \in F G \Delta\left(G^{\prime}\right)^{2} \subseteq I_{2} .
\end{aligned}
$$

Similarmente,

$$
(g-1)(h-1)(x-1)-(g-1)(x-1)(h-1)=(g-1)[h, x]=(g-1) x h((h, x)-1) .
$$


Como $(h, x)=(x, h)^{-1}=(a, b, h)^{-1} \in \gamma_{3}(G) \subseteq\left(G^{\prime}\right)^{p}$, temos $(h, x)=x^{p t}$, para algum $t$ inteiro. Assim, $(h, x)-1=\left(x^{p t}-1\right)=\left(x^{t}-1\right)^{p} \in \Delta\left(G^{\prime}\right)^{p} \subseteq \Delta\left(G^{\prime}\right)^{2}$. Analogamente, podem ser provadas as outras congruências. Usaremos este fato nos seguintes resultados.

Com ajuda dos conceitos vistos no Capítulo 1, provamos o seguinte resultado.

Lema 5.1.7. Sejam $G$ um p-grupo finito, $x=(a, b) \in G^{\prime}$ e $v=(a-1)(b-1)$ ou $v=(a-1)^{2}$. Então, $\nu(x)=t \geq 2$ e $\omega\left(v(x-1)^{k}\right)=2+t k$. Além disso, $v(x-1)^{k} \in \Delta(G)^{2+t k} \backslash \Delta(G)^{3+t k}$.

Demonstração. Como $x \in G^{\prime} \in \Gamma_{2}(G)=D_{2}(F G)$, pela definição de altura dada na página 14 , temos $\nu(x)=t \geq 2$. Agora como $v \in \Delta(G)^{2}$ em qualquer caso, temos $\omega\left(v(x-1)^{k}\right)=$ $2+\nu(x) k=2+t k$. Como a filtração determinada por $\left\{D_{t}(F G)\right\}$ é a das potências do ideal de aumento, temos $v(x-1)^{k} \in \Delta(G)^{2+t k} \backslash \Delta(G)^{3+t k}$, pois a soma de suas alturas é menor do que $3+t k$.

\section{$5.2 \quad$ Álgebras de grupo Lie nilpotentes}

Note que a única orientação $\sigma$ que pode ser definida sobre um grupo finito de ordem ímpar é a trivial.

No seguinte lema, como $G$ é um $p$-grupo finito, a orientação definida sobre $G$ é trivial; assim a prova dada em [5, Lema 2] continua válida. Por sua importância no principal resultado desta seção, escrevemos a sua prova aqui.

Lema 5.2.1. Seja $G$ um p-grupo finito com subgrupo comutador cíclico, isto é, G' é cíclico. Então t $\left((F G)^{+}\right) \geq\left|G^{\prime}\right|+1 e \operatorname{cl}\left(\mathcal{U}^{+}(F G)\right) \geq\left|G^{\prime}\right|$.

Demonstração. Sejam $a$ e $b$ em $G$ tais que $x=(a, b)$ e $\langle x\rangle=G^{\prime}$. Primeiro vamos provar que para $n \geq 2$, existem $z_{n} \in \gamma^{n}\left((F G)^{+}\right)$e $z_{n}^{\prime} \in \gamma_{n}\left(\left(\mathcal{U}^{+}(F G)\right)\right)$ tais que

$$
z_{n} \equiv z_{n}^{\prime}-1 \equiv \begin{cases}\alpha_{n}(a-1)^{2}(x-1)^{n-1}\left(\bmod I_{n}\right) & \text { se } n \text { é ímpar } \\ \alpha_{n}(a-1)(b-1)(x-1)^{n-1} \quad\left(\bmod I_{n}\right) & \text { se } n \text { é par }\end{cases}
$$

onde $\alpha_{n} \in F \backslash\{0\}$. 
Para $n \geq 1$ seja

$$
u_{n} \equiv \begin{cases}(a-1)\left(a^{-1}-1\right) & \text { se } n \text { é ímpar; } \\ (b-1)\left(b^{-1}-1\right) & \text { se } n \text { é par. }\end{cases}
$$

Dado que $a$ e $b$ são $p$-elementos, $u_{i}$ é nilpotente e assim é fácil ver que $1+u_{i}$ é uma unidade e $\varepsilon\left(\left(1+u_{i}\right)^{-1}\right)=1$.

Pelas equações (5.2)

$$
\begin{aligned}
{\left[u_{1}, u_{2}\right]=(a-1)(b-1)\left[a^{-1}, b^{-1}\right]+} & (a-1)\left[a^{-1}, b\right]\left(b^{-1}-1\right)+ \\
& (b-1)\left[a, b^{-1}\right]\left(a^{-1}-1\right)+[a, b]\left(b^{-1}-1\right)\left(a^{-1}-1\right) .
\end{aligned}
$$

De (5.6) obtemos

$$
\begin{aligned}
{\left[u_{1}, u_{2}\right] } & \\
\equiv & (a-1)(b-1) b^{-1} a^{-1}(x-1)-(a-1) b a^{-1}(x-1)\left(b^{-1}-1\right)- \\
& \quad(b-1) b^{-1} a(x-1)\left(a^{-1}-1\right)+b a(x-1)\left(b^{-1}-1\right)\left(a^{-1}-1\right) \quad\left(\bmod F G \Delta\left(G^{\prime}\right)^{2}\right)
\end{aligned}
$$

Da equação (5.5), resultam as congruências

$$
\begin{aligned}
& a^{-1}-1 \equiv-(a-1) \quad\left(\bmod \Delta(G)^{2}\right) \quad \text { e } \\
& b^{-1}-1 \equiv-(b-1) \quad\left(\bmod \Delta(G)^{2}\right)
\end{aligned}
$$

Desta forma, os quatro termos na equação (5.8) são congruentes a $(a-1)(b-1)(x-1)$ módulo $I_{2}$. Então

$$
\left[u_{1}, u_{2}\right] \equiv 4(a-1)(b-1)(x-1) \quad\left(\bmod I_{2}\right)
$$

Além disso,

$$
\begin{aligned}
\left(1+u_{1}, 1+u_{2}\right) & =1+\left(1+u_{1}\right)^{-1}\left(1+u_{2}\right)^{-1}\left[u_{1}, u_{2}\right] \\
& =1+\left(\left(1+u_{1}\right)^{-1}\left(1+u_{2}\right)^{-1}-1\right)\left[u_{1}, u_{2}\right]+\left[u_{1}, u_{2}\right] \\
& \equiv 1+4(a-1)(b-1)(x-1) \quad\left(\bmod I_{2}\right)
\end{aligned}
$$


pois $\left(\left(1+u_{1}\right)^{-1}\left(1+u_{2}\right)^{-1}-1\right)\left[u_{1}, u_{2}\right] \in \Delta(G)^{3} \Delta\left(G^{\prime}\right) \subseteq I_{2}$. Desta forma, tomando $z_{2}=$ $\left[u_{1}, u_{2}\right], z_{2}^{\prime}=\left(1+u_{1}, 1+u_{2}\right)$ e $\alpha_{2}=4$, provamos a afirmação (5.7), para $n=2$. Assuma a veracidade de (5.7), para algum $i \geq 2$, isto é, existem $w, w^{\prime} \in I_{i}$ e $\alpha_{i} \in F \backslash\{0\}$ tais que $z_{i}=\alpha_{i} v_{i}(x-1)^{i-1}+w \in \gamma^{i}\left((F G)^{+}\right)$e $1+z_{i}+w^{\prime} \in \gamma_{i}\left(\mathcal{U}^{+}(F G)\right)$, onde $v_{i}=(a-1)^{2}$ ou $v_{i}=(a-1)(b-1)$ dependendo se $i$ é ímpar ou par, respectivamente. Do Lema 5.1.4, temos

$$
\begin{aligned}
{\left[z_{i}, u_{i+1}\right] } & =\left[\alpha_{i} v_{i}(x-1)^{i-1}+w, u_{i+1}\right]=\left[\alpha_{i} v_{i}(x-1)^{i-1}, u_{i+1}\right]+\left[w, u_{i+1}\right] \\
& =\alpha_{i} v_{i}\left[(x-1)^{i-1}, u_{i+1}\right]+\alpha_{i}\left[v_{i}, u_{i+1}\right](x-1)^{i-1}+\left[w, u_{i+1}\right]
\end{aligned}
$$

Como $\left[(x-1)^{i-1}, u_{i+1}\right] \in\left[\Delta\left(G^{\prime}\right)^{i-1}, \Delta(G)^{2}\right] \subseteq \Delta(G) \Delta\left(G^{\prime}\right)^{i}$, temos

$$
\alpha_{i} v_{i}\left[(x-1)^{i-1}, u_{i+1}\right] \in \Delta(G)^{3} \Delta\left(G^{\prime}\right)^{i} \subseteq I_{i+1}
$$

Além disso, $\left[w, u_{i+1}\right] \in\left[I_{i}, \Delta(G)^{2}\right]$ e do Lema 5.1.4

$$
\begin{aligned}
{\left[I_{i}, \Delta(G)^{2}\right] } & =\left[\Delta(G)^{3} \Delta\left(G^{\prime}\right)^{i-1}+F G \Delta\left(G^{\prime}\right)^{i}, \Delta(G)^{2}\right] \\
& =\left[\Delta(G)^{3} \Delta\left(G^{\prime}\right)^{i-1}, \Delta(G)^{2}\right]+\left[F G \Delta\left(G^{\prime}\right)^{i}, \Delta(G)^{2}\right] \\
& \subseteq \Delta(G)^{3} \Delta\left(G^{\prime}\right)^{i}+F G \Delta\left(G^{\prime}\right)^{i+1}=I_{i+1} .
\end{aligned}
$$

Em consequência,

$$
\left[z_{i}, u_{i+1}\right] \equiv \alpha_{i}\left[v_{i}, u_{i+1}\right](x-1)^{i} \quad\left(\bmod I_{i+1}\right)
$$

Por meio da congruência (5.6) calculamos o colchete de Lie $\left[v_{i}, u_{i+1}\right]$, dependendo da paridade de $i$

- $i$ é par

$$
\begin{aligned}
{\left[v_{i}, u_{i+1}\right] } & =\left[(a-1)(b-1),(a-1)\left(a^{-1}-1\right)\right] \\
& =(a-1)^{2}\left[b, a^{-1}\right]+(a-1)[b, a]\left(a^{-1}-1\right) \\
& \equiv 2(a-1)^{2}(x-1) \quad\left(\bmod I_{2}\right),
\end{aligned}
$$


- $i$ é ímpar,

$$
\begin{aligned}
{\left[v_{i}, u_{i+1}\right]=} & {\left[(a-1)^{2},(b-1)\left(b^{-1}-1\right)\right] } \\
= & (a-1)(b-1)\left[a, b^{-1}\right]+(a-1)[a, b]\left(b^{-1}-1\right)+ \\
& (b-1)\left[a, b^{-1}\right](a-1)+[a, b]\left(b^{-1}-1\right)(a-1) \\
\equiv & -4(a-1)(b-1)(x-1) \quad\left(\bmod I_{2}\right) .
\end{aligned}
$$

Logo obtemos

$$
\left[z_{i}, u_{i+1}\right] \equiv \alpha_{i+1} v_{i+1}(x-1)^{i} \quad\left(\bmod I_{i+1}\right)
$$

onde $\alpha_{i+1}=-4 \alpha_{i}$ se $i$ é ímpar ou $\alpha_{i+1}=2 \alpha_{i}$, se $i$ é par. Além disso,

$$
\begin{aligned}
& \left(1+z_{i}+w^{\prime}, 1+u_{i+1}\right) \\
& =1+\left(1+z_{i}+w^{\prime}\right)^{-1}\left(1+u_{i+1}\right)^{-1}\left[z_{i}+w^{\prime}, u_{i+1}\right] \\
& =1+\left(\left(1+z_{i}+w^{\prime}\right)^{-1}\left(1+u_{i+1}\right)^{-1}-1\right)\left[z_{i}+w^{\prime}, u_{i+1}\right]+\left[z_{i}, u_{i+1}\right]+\left[w^{\prime}, u_{i+1}\right] \\
& \equiv 1+\alpha_{i+1} v_{i+1}(x-1)^{i} \quad\left(\bmod I_{i+1}\right)
\end{aligned}
$$

Logo, é suficiente tomar $z_{i+1}=\left[z_{i}, u_{i+1}\right]$ e $z_{i+1}^{\prime}=\left(1+z_{i}+w^{\prime}, 1+u_{i+1}\right)$. Em conclusão, a afirmação (5.7) é verdadeira, para todo $n \geq 2$.

Mostraremos que $z_{m}$ não é zero, para $m=\left|G^{\prime}\right|$. É suficiente mostrar que $\gamma=v_{m}(x-1)^{m-1}$ não pertence a $I_{m}$. Como $m=\left|G^{\prime}\right|$ é uma potência de $p$, temos $F G \Delta\left(G^{\prime}\right)^{m}=0$ e $I_{m}=$ $\Delta(G)^{3} \Delta\left(G^{\prime}\right)^{m-1}$. Pelo Lema 5.1.7, $\omega(\gamma)=2+t(m-1)$, com $\nu(x)=t \geq 2$, o que significa $\gamma \in \Delta(G)^{2+t(m-1)} \backslash \Delta(G)^{3+t(m-1)}$. Como $\Delta(G)^{i}$ tem uma $F$-base de elementos regulares de peso maior ou igual a $i$, temos a inclusão $\Delta(G)^{3} \Delta\left(G^{\prime}\right)^{m-1} \subseteq \Delta(G)^{3+t(m-1)}$. Em conclusão, $\gamma$ não pertence a $I_{m}$.

Assim podemos estender o Teorema 1 em [5] ao caso quando $F G$ tem uma involução clássica orientada.

Teorema 5.2.2. Seja FG uma álgebra de grupo Lie nilpotente de característica ímpar com uma involução clássica orientada, com orientação não trivial. Então $\mathrm{t}\left((F G)^{+}\right)=\left|G^{\prime}\right|+1$ se, 
e somente se, $G^{\prime}$ é cíclico. Além disso, quando $G$ é um grupo de torção, $\operatorname{cl}\left(\mathcal{U}^{+}(F G)\right)=\left|G^{\prime}\right|$ se, e somente se, $G^{\prime}$ é cíclico.

Demonstração. Seja FG uma álgebra de grupo Lie nilpotente. Suponha primeiro $\mathrm{t}\left((F G)^{+}\right)=\left|G^{\prime}\right|+1$. Como $G^{\prime}$ é um p-grupo finito, se $G^{\prime}$ não é cíclico, de [6], temos $\mathrm{t}\left((F G)^{+}\right) \leq \mathrm{t}^{\mathrm{L}}(F G)<\left|G^{\prime}\right|+1$, o que leva à desigualdade $\mathrm{t}\left((F G)^{+}\right)<\left|G^{\prime}\right|+1$. Claramente obtemos uma contradição, portanto $G^{\prime}$ deve ser cíclico.

Reciprocamente, suponha $G^{\prime}$ cíclico. Como $F G$ é Lie nilpotente, $G$ é um grupo nilpotente $p$-abeliano e por [3, Lema 1] existe um $p$-grupo finito $P$ que é isomorfo a um subgrupo de um grupo fator de $G$ e tal que $P^{\prime} \simeq G^{\prime}$. Da prova de [3, Lema 1], temos $P \simeq H / A$, onde $A$ é um subgrupo central, maximal, livre de torção.

Assuma que existe $g \in A$ tal que $\sigma(g)=-1$. Desta forma, como $G=N \cup g N, G^{\prime}=N^{\prime}$. Usando em FP a involução clássica, os Lemas 5.2.1 e 5.1.1 implicam

$$
\left|G^{\prime}\right|+1=\left|N^{\prime}\right|+1=\left|P^{\prime}\right|+1 \leq \mathrm{t}\left((F P)^{+}\right) \leq \mathrm{t}\left((F N)^{+}\right) \leq \mathrm{t}\left((F G)^{+}\right) .
$$

Por outro lado, se $A \subseteq N$, usando em $F P \simeq F(H / A)$ a involução clássica orientada induzida como no Lema 5.1.1, novamente os lemas 5.2.1 e 5.1.1, levam a

$$
\left|G^{\prime}\right|+1=\left|P^{\prime}\right|+1 \leq \mathrm{t}\left((F P)^{+}\right) \leq \mathrm{t}\left((F G)^{+}\right) .
$$

A prova da segunda parte é similar.

O Teorema anterior implica que se $F G$ é Lie nilpotente, $\operatorname{car}(F)>2$ e t $(F G)=\left|G^{\prime}\right|+1$, então $\mathrm{t}\left((F G)^{+}\right)=\mathrm{t}(F G)$.

\subsection{Grupos que contêm uma cópia de $Q_{8}$}

A partir daqui vamos supor $Q_{8}=\left\langle x, y: x^{4}=1, x^{2}=y^{2}, x^{y}=x^{-1}\right\rangle \subseteq G$. Suponha agora que $(F G)^{+}$é Lie nilpotente. Como $G$ contém uma cópia de $Q_{8}$ e $F Q_{8}$ não é Lie nilpotente, pelo Teorema 2.1.2, a álgebra de grupo $F G$ não pode ser Lie nilpotente. As álgebras de grupo deste tipo foram caracterizadas no Teorema 4.1.6. Desta forma $G \simeq\left\langle Q_{8}, g\right\rangle \times E \times P$, 
onde $E^{2}=1, g \in G \backslash N$ é tal que $(g, x)=(g, y)=1$ e $g^{2}=x^{2}$ e $P$ é um $p$-grupo finito. A partir daqui, $G \simeq\left\langle Q_{8}, g\right\rangle \times E \times P$ denota o grupo com as relações anteriores. Na demonstração deste resultado foi provada a desigualdade $\mathrm{t}\left((F G)^{+}\right) \leq|P|+1$. Quando $P$ é trivial ou a característica de $F$ é $0, G \simeq\left\langle Q_{8}, g\right\rangle \times E$, pelo Lema 4.1.4, $(F G)^{+}$é comutativo e assim $\mathrm{t}\left((F G)^{+}\right)=2=|P|+1$. No restante deste capítulo assumimos que $P$ é não trivial e $\operatorname{car}(F)=p>2$.

Antes de dar o resultado principal desta seção necessitamos apresentar alguns lemas técnicos.

Lema 5.3.1. Considere a álgebra de grupo FG com uma involução clássica orientada. Então

$$
\left((F G)^{+}\right)^{(n)} \subseteq F G \Delta(P)^{n}
$$

para todo $n \geq 2$.

Demonstração. Lembramos que os elementos simétricos são gerados como um $F$-módulo pelo conjunto $\mathcal{S}=\left\{z+z^{-1}: z \in N\right\} \cup\left\{z-z^{-1}: z \in G \backslash N\right\}$. Se $z \in N$, então $z=$ ah com $a \in Q_{8} \times E$ e $h \in P$. Se $a^{2} h=1$, então $h=1$ e $a^{2}=1$. Assim, $a \in \zeta\left(Q_{8} \times E\right)$. Assumamos que $a^{2} h \neq 1$ e consideremos os seguintes casos:

1. Se $a^{2}=1$ e $h \neq 1$, então $z+z^{-1}=a h+a^{-1} h^{-1}=a\left(h+h^{-1}\right)$.

2. Se $a^{2} \neq 1$ e $h=1$, então $z+z^{-1}=a h+a^{-1} h^{-1}=a+a^{-1}$.

3. Se $a^{2} \neq 1$ e $h \neq 1$, então $z+z^{-1}=a h+a^{-1} h^{-1}=a h+a^{3} h^{-1}=a\left(h+a^{2} h^{-1}\right)$.

Por outro lado, se $z \in G \backslash N$, podemos escrever $z=g a h$, com $a \in Q_{8} \times E$ e $h \in P$. Se $a^{2} h=1$, então $a^{2}=h=1$. Novamente $a \in \zeta\left(Q_{8} \times E\right)$. Desta forma, $z-z^{-1}=g a h-g^{-1} a^{-1} h^{-1}=$ $g a-g^{-1} a=g a\left(1-g^{2}\right)$. Assumamos que $a^{2} h \neq 1$ e consideremos os seguintes casos:

1. Se $a^{2}=1$ e $h \neq 1$, então $z-z^{-1}=g a h-g^{-1} a^{-1} h^{-1}=a g\left(h-g^{2} h^{-1}\right)$.

2. Se $a^{2} \neq 1$ e $h=1$, então $z-z^{-1}=g a h-g^{-1} a^{-1} h^{-1}=g a-g^{3} a^{3}=g a-g a=0$.

3. Se $a^{2} \neq 1$ e $h \neq 1$, então $z-z^{-1}=g a h-g^{-1} a^{-1} h^{-1}=a g h-a^{3} g^{3} h^{-1}=a g\left(h-h^{-1}\right)$, pois $a^{3} g^{3}=a g$. 
Das considerações anteriores, obtemos que:

$$
\mathcal{S}=\mathcal{A} \cup \mathcal{B} \cup \mathcal{C} \cup \mathcal{D} \cup \mathcal{E} \cup \zeta\left(Q_{8} \times E\right)
$$

onde

$$
\begin{aligned}
& \mathcal{A}=\left\{a\left(h+a^{2} h^{-1}\right): a \in Q_{8} \times E, h \in P \text { e }\left(a^{2} \neq 1 \text { e } h \neq 1\right)\right\}, \\
& \mathcal{B}=\left\{a\left(h+h^{-1}\right): a \in Q_{8} \times E, h \in P \text { e }\left(a^{2}=1 \text { e } h \neq 1\right)\right\}, \\
& \mathcal{C}=\left\{a+a^{-1}: a \in Q_{8} \times E, h \in P \text { e }\left(a^{2} \neq 1 \text { e } h=1\right)\right\}, \\
& \mathcal{D}=\left\{a g\left(h-g^{2} h^{-1}\right): a \in Q_{8} \times E, h \in P \text { e }\left(a^{2}=1 \text { e } h \neq 1\right)\right\}, \\
& \mathcal{E}=\left\{a g\left(h-h^{-1}\right): a \in Q_{8} \times E, h \in P \text { e }\left(a^{2} \neq 1 \text { e } h \neq 1\right)\right\} .
\end{aligned}
$$

Dado $a \in Q_{8} \times E$ e $h \in P$ tais que $a^{2} \neq 1$ e $h \neq 1$, temos $1+a^{2}$ é simétrico, com $a^{2} \in \zeta\left(Q_{8} \times E\right)$. Desta forma,

$$
a\left(h+a^{2} h^{-1}\right)+1+a^{2}=a(h-1)+a^{3}\left(h^{-1}-1\right)+1+a+a^{2}+a^{3},
$$

onde $1+a+a^{2}+a^{3}$ é um elemento central em $F G$ e $a(h-1)+a^{3}\left(h^{-1}-1\right) \in F G \Delta(P)$. Claramente $a g\left(h-h^{-1}\right) \in F G \Delta(P)$. Além disso, se $a^{2}=1$ e $h \neq 1$, então $a g\left(h-g^{2} h^{-1}\right)=$ $a g(h-1)-a g^{3}\left(h^{-1}-1\right)+a\left(g-g^{-1}\right) \in F G \Delta(P)+\zeta(F G)$.

Assim o conjunto

$$
\widetilde{\mathcal{S}}=\mathcal{A}^{\prime} \cup \mathcal{B} \cup \mathcal{C} \cup \mathcal{D} \cup \mathcal{E} \cup \zeta\left(Q_{8} \times E\right)
$$

também gera $(F G)^{+}$como um $F$-módulo, onde

$$
\mathcal{A}^{\prime}=\left\{a\left(h+a^{2} h^{-1}\right)+1+a^{2}: a \in Q_{8} \times E, h \in P \text { e }\left(a^{2} \neq 1 \text { e } h \neq 1\right)\right\}
$$

e $\mathcal{B}, \mathcal{C}, \mathcal{D}$ e $\mathcal{E}$ são como acima. Por conseguinte,

$$
(F G)^{+} \subseteq F G \Delta(P)+\zeta(F G)
$$


A prova segue por indução sobre $n$. Com efeito, se $n=2$, temos

$$
\left[(F G)^{+},(F G)^{+}\right] \subseteq[F G \Delta(P), F G \Delta(P)] \subseteq F G \Delta(P)^{2}
$$

Suponha que o lema vale para algum $n \geq 2$. Tome $\alpha \in\left((F G)^{+}\right)^{(n)}$ e $\beta \in(F G)^{+}$. Logo

$$
[\alpha, \beta] \in\left[F G \Delta(P)^{n}, F G \Delta(P)\right] \subseteq F G \Delta(P)^{n+1}
$$

e obtemos $\left((F G)^{+}\right)^{(n+1)} \subseteq F G \Delta(P)^{n+1}$, como queríamos provar.

No que segue, denotamos com $c=x^{2}$, o elemento central de $Q_{8} \times E$, tal que $\left(Q_{8} \times E\right)^{2}=$ $\langle c\rangle$. Dado $n \geq 2$, denotamos com $M_{n}$ o $F$-subespaço de $F G$ gerado pelo conjunto

$$
\left\{\left(h_{1}-h_{1}^{-1}\right) \cdots\left(h_{n}-h_{n}^{-1}\right)(1-c) a: h_{1}, \ldots, h_{n} \in P, a \in\left(Q_{8} \times E\right) \backslash \zeta\left(Q_{8} \times E\right)\right\} .
$$

Para abreviar, escrevemos $f_{1, \ldots, n}=\left(h_{1}-h_{1}^{-1}\right) \cdots\left(h_{n}-h_{n}^{-1}\right)$.

Sejam $S_{n}$ o grupo simétrico de grau $n$ e $F S_{n}$ sua álgebra de grupo sobre o corpo $F$. Definimos uma ação de grupo de $S_{n}$ sobre $M_{n}$ como segue: para cada elemento $\sigma \in S_{n}$ e cada gerador $f_{1, \ldots, n}(1-c) a$ de $M_{n}$ definimos

$$
\sigma \cdot f_{1, \ldots, n}(1-c) a=f_{\sigma(1), \ldots, \sigma(n)}(1-c) a
$$

É possível estender linearmente esta ação de grupo de $S_{n}$ sobre o conjunto gerador a todo o subespaço $M_{n}$. Do mesmo modo, estendemos esta ação de grupo a uma ação da álgebra de grupo $F S_{n}$ sobre $M_{n}$ : para $x=\sum_{\sigma \in S_{n}} \alpha_{\sigma} \sigma \in F S_{n}$ e $z \in M_{n}$, definimos

$$
x \cdot z=\sum_{\sigma \in S_{n}} \alpha_{\sigma}(\sigma \cdot z)
$$

Para $n \geq 2$, definimos os elementos $x_{2, n}, x_{3, n}, \ldots, x_{n, n}$ de $F S_{n}$ em forma recursiva:

$$
\begin{aligned}
& x_{2, n}=1+(2,1), \\
& x_{i, n}=x_{i-1, n}+x_{i-1, n}(i, i-1, \ldots, 1), \text { para } 3 \leq i \leq n .
\end{aligned}
$$


Lema 5.3.2. Para todo $n \geq 2$, tem-se $x_{n, n} M_{n} \in \gamma^{n}\left((F G)^{+}\right)(1-c)$.

Demonstração. Seja $a \in\left(Q_{8} \times E\right) \backslash \zeta\left(Q_{8} \times E\right)$. Assim existem $a_{1}, a_{2} \in N$ tais que $a=a_{1} a_{2}$ e $\left(a_{1}, a_{2}\right) \neq 1$. Dados $h_{1}, h_{2} \in P$, temos

$$
\begin{array}{r}
{\left[a_{1}\left(h_{1}+a_{1}^{2} h_{1}^{-1}\right), a_{2}\left(h_{2}+a_{2}^{2} h_{2}^{-1}\right)\right](1-c)=a_{1} a_{2}\left(h_{1}+a_{1}^{2} h_{1}^{-1}\right)\left(h_{2}+a_{2}^{2} h_{2}^{-1}\right)(1-c)-} \\
\left(h_{2}+a_{2}^{2} h_{2}^{-1}\right)\left(h_{1}+a_{1}^{2} h_{1}^{-1}\right) a_{2} a_{1}(1-c) .
\end{array}
$$

Agora, somando e subtraindo $a_{1} a_{2}\left(h_{2}+a_{2}^{2}+h_{2}^{-1}\right)\left(h_{1}+a_{1} h_{1}^{-1}\right)(1-c)$, a igualdade (5.12) transforma-se em

$$
\begin{aligned}
& {\left[a_{1}\left(h_{1}+a_{1}^{2} h_{1}^{-1}\right), a_{2}\left(h_{2}+a_{2}^{2} h_{2}^{-1}\right)\right](1-c)} \\
& =a_{1} a_{2}\left[h_{1}+a_{1}^{2} h_{1}^{-1}, h_{2}+a_{2}^{2} h_{2}^{-1}\right](1-c)+\left(h_{2}+a_{2}^{2} h_{2}^{-1}\right)\left(h_{1}+a_{1}^{2} h_{1}^{-1}\right)\left[a_{1}, a_{2}\right](1-c) .
\end{aligned}
$$

Como $a_{1}^{2}=a_{2}^{2}=c$, segue

$$
\begin{aligned}
& a_{1} a_{2}\left[h_{1}+a_{1}^{2} h_{1}^{-1}, h_{2}+a_{2}^{2} h_{2}^{-1}\right](1-c)=a_{1} a_{2}\left[h_{1}+c h_{1}^{-1}, h_{2}+c h_{2}^{-1}\right](1-c) \\
& =a_{1} a_{2}\left(\left(h_{1} h_{2}+c h_{1} h_{2}^{-1}+c h_{1}^{-1} h_{2}+h_{1}^{-1} h_{2}^{-1}\right)-\right. \\
& \left.\quad\left(h_{2} h_{1}+c h_{2} h_{1}^{-1}+c h_{2}^{-1} h_{1}+h_{2}^{-1} h_{1}^{-1}\right)\right)(1-c) \\
& =a_{1} a_{2}\left(h_{1}-h_{1}^{-1}\right)\left(h_{2}-h_{2}^{-1}\right)(1-c)-\left(h_{2}-h_{2}^{-1}\right)\left(h_{1}-h_{1}^{-1}\right) a_{1} a_{2}(1-c) \\
& =\left(h_{1}-h_{1}^{-1}\right)\left(h_{2}-h_{2}^{-1}\right)(1-c) a-\left(h_{2}-h_{2}^{-1}\right)\left(h_{1}-h_{1}^{-1}\right)(1-c) a .
\end{aligned}
$$

Similarmente, como $a_{2} a_{1} c=a_{1} a_{2}$, temos a igualdade $\left[a_{1}, a_{2}\right](1-c)=2 a_{1} a_{2}(1-c)$, donde

$$
\begin{aligned}
& \left(h_{2}+a_{2}^{2} h_{2}^{-1}\right)\left(h_{1}+a_{2}^{2} h_{1}^{-1}\right)\left[a_{1}, a_{2}\right](1-c)=\left(h_{2}+c h_{2}^{-1}\right)\left(h_{1}+c h_{1}^{-1}\right)\left[a_{1}, a_{2}\right](1-c) \\
& =\left(h_{2} h_{1}+c h_{2} h_{1}^{-1}+c h_{2}^{-1} h_{1}+h_{2}^{-1} h_{1}^{-1}\right)\left[a_{1}, a_{2}\right](1-c) \\
& =2\left(h_{2}-h_{2}^{-1}\right)\left(h_{1}-h_{1}^{-1}\right)(1-c) a .
\end{aligned}
$$

Desta forma, substituindo as igualdades (5.14) e (5.15) na equação (5.13), obtemos

$$
\begin{aligned}
{\left[a_{1}\left(h_{1}+a_{1}^{2} h_{1}^{-1}\right), a_{2}\right.} & \left.\left(h_{2}+a_{2}^{2} h_{2}^{-1}\right)\right](1-c) \\
& =\left(h_{1}-h_{1}^{-1}\right)\left(h_{2}-h_{2}^{-1}\right)(1-c) a+\left(h_{2}-h_{2}^{-1}\right)\left(h_{1}-h_{1}^{-1}\right)(1-c) a
\end{aligned}
$$


Em consequência,

$$
\begin{aligned}
& x_{2,2} f_{1,2}(1-c) a=\left(h_{1}-h_{1}^{-1}\right)\left(h_{2}-h_{2}^{-1}\right)(1-c) a+\left(h_{2}-h_{2}^{-1}\right)\left(h_{1}-h_{1}^{-1}\right)(1-c) a \\
& =\left[a_{1}\left(h_{1}+a_{1}^{2} h_{1}^{-1}\right), a_{2}\left(h_{2}+a_{2}^{2} h_{2}^{-1}\right)\right](1-c) \in \gamma^{2}\left((F G)^{+}\right)(1-c),
\end{aligned}
$$

o que prova o lema para $n=2$.

Assuma $x_{n-1, n-1} M_{n-1} \subseteq \gamma^{n-1}\left((F G)^{+}\right)(1-c)$. Sejam $h_{1}, \ldots, h_{n} \in P, a \in\left(Q_{8} \times E\right) \backslash$ $\zeta\left(Q_{8} \times E\right)$. Escolha elementos $a_{1}, a_{2} \in Q_{8} \times E$ tais que $a_{1} a_{2}=a$ e $\left(a_{1}, a_{2}\right) \neq 1$. Então

$$
\begin{aligned}
& x_{n, n} f_{1, \ldots, n}(1-c) a=x_{n-1, n} f_{1, \ldots, n}(1-c) a+x_{n-1, n}(n, n-1, \ldots, 1) f_{1, \ldots, n}(1-c) a \\
& =x_{n-1, n-1}\left(f_{1, \ldots, n-1}(1-c) a\right)\left(h_{n}-h_{n}^{-1}\right)+\left(h_{n}-h_{n}^{-1}\right) x_{n-1, n-1} f_{1, \ldots, n-1}(1-c) a \\
& =x_{n-1, n-1}\left(f_{1, \ldots, n-1}(1-c) a\right)\left(h_{n}-h_{n}^{-1}\right)-\left(h_{n}+c h_{n}^{-1}\right) x_{n-1, n-1}\left(f_{1, \ldots, n-1}(1-c) a_{2} a_{1}\right) \\
& =\left[x_{n-1, n-1} f_{1, \ldots, n-1}(1-c) a_{1},\left(h_{n}+a_{2}^{2} h_{n}^{-1}\right) a_{2}\right] .
\end{aligned}
$$

A última igualdade implica

$$
x_{n, n} M_{n} \subseteq\left[x_{n-1, n-1} M_{n-1},(F G)^{+}\right] \subseteq\left[\gamma^{n-1}\left((F G)^{+}\right)(1-c),(F G)^{+}\right]=\gamma^{n}\left((F G)^{+}\right)(1-c)
$$

Antes de enunciar o próximo lema, lembramos o seguinte fato.

Proposição 5.3.3. Sejam $P$ um p-grupo finito, $H$ um subgrupo normal maximal de $P$, de indice $p$, e $s \in P \backslash H$. Então $(s-1)^{p-1} \widehat{H}=\widehat{P}$.

Demonstração. Como $P$ é a união disjunta das classe laterais $H, s H, \ldots, s^{p-1} H$, segue que

$$
\begin{aligned}
(s-1)^{p-1} \widehat{H} & =\left(1+s+\cdots+s^{p-1}\right) \widehat{H} \\
& =\widehat{H}+s \widehat{H}+\cdots+s^{p-1} \widehat{H}=\widehat{P} .
\end{aligned}
$$

Lema 5.3.4. Se $|P|=p^{k}$, então $\widehat{P}(1-c) a \in \gamma^{k(p-1)}\left((F G)^{+}\right)$, para algum $a \in Q_{8} \times E$ 
Demonstração. Primeiro provamos que são satisfeitas duas igualdades. Sejam $a, b \in\left(Q_{8} \times E\right) \backslash \zeta\left(Q_{8} \times E\right)$ e $h_{1}, h_{2} \in P$ tais que $(a, b) \neq 1$ e $\left(h_{1}, h_{2}\right)=1$. Então

$$
\begin{aligned}
{\left[a\left(h_{1}+a^{2} h_{1}^{-1}\right), b\left(h_{2}+b^{2} h_{2}^{-1}\right)\right] } & =\left(h_{2}+b^{2} h_{2}^{-1}\right)\left(h_{1}+a^{2} h_{1}^{-1}\right)[a, b] \\
& =\left(h_{2}-h_{2}^{-1}\right)\left(h_{1}-h_{1}^{-1}\right)(1-c) a b
\end{aligned}
$$

Além disso, se $\alpha \in F P, h \in P$ e $a, b \in\left(Q_{8} \times E\right) \backslash \zeta\left(Q_{8} \times E\right)$ são tais que $[\alpha, h]=0$ e $(a, b) \neq 1$, então

$$
\begin{aligned}
{\left[\alpha(1-c) a, b\left(h+b^{2} h^{-1}\right)\right] } & =\alpha(1-c)\left(h+b^{2} h^{-1}\right)[a, b] \\
& =\alpha\left(h-h^{-1}\right)(1-c)^{2} a b=2 \alpha\left(h-h^{-1}\right)(1-c) a b .
\end{aligned}
$$

Assuma $P=\langle h\rangle$, onde $h$ é de ordem $p$ e sejam $a_{1}, a_{2}, \ldots, a_{p-1} \in\left(Q_{8} \times E\right) \backslash \zeta\left(Q_{8} \times E\right)$ tais que $\left(a_{1} a_{2} \cdots a_{i-1}, a_{i}\right) \neq 1$, para todo $1<i<p$. Usando (5.17) e (5.18) podemos calcular o colchete de Lie

$$
\begin{array}{ll}
{\left[a_{1}\left(h+a_{1}^{2} h^{-1}\right), a_{2}\left(h+a_{2}^{2} h^{-1}\right), \ldots, a_{p-1}\left(h+a_{p-1}^{2} h^{-1}\right)\right]} & \\
=\left[\left(h-h^{-1}\right)^{2}(1-c) a_{1} a_{2}, a_{3}\left(h+a_{3}^{2} h^{-1}\right), \ldots, a_{p-1}\left(h+a_{p-1}^{2} h^{-1}\right)\right] & (\text { por }(5.17)) \\
=\left[2\left(h-h^{-1}\right)^{3}(1-c) a_{1} a_{2} a_{3}, \ldots, a_{p-1}\left(h+a_{p-1}^{2} h^{-1}\right)\right] & (\text { por }(5.18)) \\
\vdots & \\
=2^{p-3}\left(h-h^{-1}\right)^{p-1}(1-c) a_{1} \cdots a_{p-1} . &
\end{array}
$$

Como $\left(h-h^{-1}\right)^{p-1}=\left(h^{2}-1\right)^{p-1} h^{-(p-1)}=\widehat{\langle h\rangle}=\widehat{P}$ e $2^{p-3} \neq 0$, temos $\widehat{P}(1-c) a_{1} \cdots a_{p-1} \in \gamma^{p-1}\left((F G)^{+}\right)$.

Suponha que $P$ é de ordem $p^{k}$, com $k>1$, e que o lema é verdadeiro para grupos de ordem $p^{k-1}$. Então $P$ tem um subgrupo normal maximal $H$ de índice $p$. Por indução, existe $b_{0} \in\left(Q_{8} \times E\right) \backslash \zeta\left(Q_{8} \times E\right)$ tal que

$$
\widehat{H}(1-c) b_{o} \in \gamma^{(k-1)(p-1)}\left(\left(F\left(Q_{8} \times E \times H\right)\right)^{+}\right) \subseteq \gamma^{(k-1)(p-1)}\left((F G)^{+}\right) .
$$

Como $H$ é um subgrupo normal de $P$, do Lema 3.4.3 em [30], sabemos que $\widehat{H}$ é central em $F G$. 
Podemos escolher $b_{1}, b_{2}, \ldots, b_{p-1} \in\left(Q_{8} \times E\right) \backslash \zeta\left(Q_{8} \times E\right)$ tais que $\left(b_{0} b_{1} \cdots b_{i-1}, b_{i}\right) \neq 1$ para todo $1<i<p$. Para $s \in P \backslash H$ e $b_{i}\left(s+b_{i}^{2} s^{-1}\right) \in \mathcal{S}$, usando a equação (5.18) e a Proposição 5.3.3 podemos calcular o colchete de Lie

$$
\begin{aligned}
{\left[\widehat{H}(1-c) b_{o}, b_{1}\left(s+b_{1}^{2} s^{-1}\right), \ldots, b_{p-1}(s+\right.} & \left.\left.b_{p-1}^{2} s^{-1}\right)\right] \\
& =2^{p-1} \widehat{H}\left(s-s^{-1}\right)^{p-1}(1-c) b_{o} b_{1} \cdots b_{p-1} \\
& =2^{p-1} \widehat{H}\left(s^{2}-1\right)^{p-1} s^{-(p-1)}(1-c) b_{o} b_{1} \cdots b_{p-1} \\
& =2^{p-1} \widehat{P} s^{-(p-1)}(1-c) b_{o} b_{1} \cdots b_{p-1} \\
& =2^{p-1} \widehat{P}(1-c) b_{o} b_{1} \cdots b_{p-1} .
\end{aligned}
$$

Assim $\widehat{P}(1-c) b_{o} b_{1} \cdots b_{p-1} \in \gamma^{k(p-1)}\left((F G)^{+}\right)$, como queríamos mostrar.

Necessitaremos as seguintes observações no próximo teorema.

Definição 5.3.5. Dizemos que um p-grupo finito $P$ é poderoso ${ }^{1}$ se $P^{\prime} \subseteq P^{p}$.

Seja $P$ um grupo poderoso. Denotamos o $i$-ésimo subgrupo dimensional por $D_{i}=$ $D_{i}(F P)$. Pelo [20, Teorema 5.5], temos $D_{1}=P$ e para $n>1$,

$$
D_{n}=\left\langle\left(D_{n-1}, P\right),\left(D_{\left\lceil\frac{n}{p}\right\rceil}\right)^{p}\right\rangle
$$

Pode-se mostrar facilmente que $\left(P^{p^{i}}\right)^{p^{j}}=P^{p^{i+j}}$ e $\left(P^{p^{i}}, P\right) \subseteq P^{p^{i+1}}$, para todo par $i, j$. Logo, se $p^{i-1}<n \leq p^{i}$, então $D_{n}=P^{p^{i}}$.

Lema 5.3.6. Sejam $P$ um p-grupo finito poderoso, $h_{i}-1 \in \Delta(P)^{k_{i}}$ e $h_{j}-1 \in \Delta(P)^{k_{j}}$, onde $k_{i}$ e $k_{j}$ são inteiros positivos. Então

$$
\left(h_{i}-1\right)\left(h_{j}-1\right) \equiv\left(h_{j}-1\right)\left(h_{i}-1\right) \quad\left(\bmod \Delta(P)^{k_{i}+k_{j}+1}\right)
$$

Demonstração. Mostramos primeiro que $\left(D_{i}, D_{j}\right) \subseteq D_{i+j+1}$, para todo par $i, j$. Sejam $h_{i} \in$ $D_{i}$ e $h_{j} \in D_{j}$. Temos a seguinte equação

$$
\left(h_{i}, h_{j}\right)-1=h_{i}^{-1} h_{j}^{-1}\left(\left(h_{i}-1\right)\left(h_{j}-1\right)-\left(h_{j}-1\right)\left(h_{i}-1\right)\right) .
$$

\footnotetext{
${ }^{1}$ Do inglês, powerful, ver [5].
} 
Se $i$ não é uma potência de $p$, então $h_{i} \in D_{i}=D_{i+1}$, logo de $(5.21)$, temos $\left(h_{i}, h_{j}\right)-1 \in$ $\Delta(P)^{i+j+1}, \operatorname{assim}\left(h_{i}, h_{j}\right) \in D_{i+j+1}$. Se tanto $i$ quanto $j$ são potências de $p$, então $i+j$ não pode ser uma potência de $p$ e assim $D_{i+j}=D_{i+j+1}$. De $(5.21)$, segue $\left(h_{i}, h_{j}\right) \in D_{i+j+1}$, portanto, nossa afirmação está provada. Se $j$ não é potência de $p$, o mesmo resultado segue de forma análoga.

Sejam $h_{i}-1 \in \Delta(P)^{k_{i}}$ e $h_{j}-1 \in \Delta(P)^{k_{j}}$, para alguns $k_{i}, k_{j}$. Então

$$
\left(h_{i}-1\right)\left(h_{j}-1\right)=\left(h_{j}-1\right)\left(h_{i}-1\right)+h_{j} h_{i}\left(\left(h_{i}, h_{j}\right)-1\right)
$$

e, como $\left(h_{i}, h_{j}\right) \in D_{k_{i}+k_{j}+1}$, segue o resultado.

Apresentamos o principal resultado desta seção, o qual estende o Teorema 2 de [5]. É bem sabido que o ideal de aumento de um $p$-grupo finito $P$ é nilpotente, ver [30, Teorema 6.3.1]. Denotamos com $\mathrm{t}_{\text {nil }}(P)$, denominado índice de nilpotência, o menor inteiro positivo $k$ tal que $\Delta(P)^{k}=0$. Pelo Corolário 4.1.9, $\mathcal{U}^{+}(F G)$ é nilpotente.

Teorema 5.3.7. Seja F um corpo de característica $p>2$. Considere a álgebra de grupo $F G$ com uma involução clássica orientada. Suponha $Q_{8} \subseteq G,(F G)^{+}$Lie nilpotente e que o p-subgrupo de Sylow P de $G$ é de ordem $p^{m}$, com $m \geq 1$. Então

(i) $1+m(p-1) \leq \mathrm{t}\left((F G)^{+}\right) \leq \mathrm{t}^{\mathrm{L}}\left((F G)^{+}\right) \leq \mathrm{t}_{\text {nil }}(P)$ e $\operatorname{cl}\left(\mathcal{U}^{+}(F G)\right) \leq \mathrm{t}_{\text {nil }}(P)-1$.

(ii) Se $\mathrm{t}\left((F G)^{+}\right)=\mathrm{t}_{\text {nil }}(P)$, então $\operatorname{cl}\left(\mathcal{U}^{+}(F G)\right)+1=\mathrm{t}\left((F G)^{+}\right)$.

(iii) Se P é poderoso, então $\mathrm{t}\left((F G)^{+}\right)=\mathrm{t}_{\text {nil }}(P)$.

(iv) Se P é abeliano, então, para todo $k \geq 2$, o $F$-espaço $\gamma^{k}\left((F G)^{+}\right)$é gerado pelo conjunto

$$
\begin{aligned}
\mathcal{M}_{k}= & \left\{\left(h_{1}-h_{1}^{-1}\right) \cdots\left(h_{k}-h_{k}^{-1}\right)\left(1-a^{2}\right) a: h_{i} \in P, a \in\left(Q_{8} \times E\right) \backslash \zeta\left(Q_{8} \times E\right)\right\} \cup \\
& \left\{g\left(h_{1}-h_{1}^{-1}\right) \cdots\left(h_{k}-h_{k}^{-1}\right)\left(1-a^{2}\right) a: h_{i} \in P, a \in\left(Q_{8} \times E\right) \backslash \zeta\left(Q_{8} \times E\right)\right\} .
\end{aligned}
$$

Demonstração. Do Lema 5.3.4, para algum a $\in \quad Q_{8} \times E$ tem-se $0 \neq \widehat{P}(1-c) a \in \gamma^{m(p-1)}\left((F G)^{+}\right)$. Desta forma, $1+m(p-1) \leq \mathrm{t}\left((F G)^{+}\right)$. Por outro lado, o Lema 5.3.1 implica $\mathrm{t}^{\mathrm{L}}\left((F G)^{+}\right) \leq \mathrm{t}_{\text {nil }}(P)$. 
Para mostrar a segunda parte do enunciado, considere os elementos simétricos $u_{i}=1-a_{i}\left(1+a_{i}^{2}\right)+x_{i}$, onde $x_{i}=a_{i}\left(h_{i}+a_{i}^{2} h_{i}^{-1}\right) \in \mathcal{S}, a_{i} \in Q_{8} \times E, h_{i} \in P$. Assim, $u_{i}=1+a_{i}\left(h_{i}-1\right)+a_{i}^{3}\left(h_{i}^{-1}-1\right) \in 1+F G \Delta(P)$. Como $F G \Delta(P)$ é um ideal nilpotente, temos $1+F G \Delta(P)$ um subgrupo normal de $\mathcal{U}(F G)$ e, em consequência, $u_{i}$ é uma unidade em FG. Provaremos, por indução a congruência

$$
\left(u_{1}, u_{2}, \ldots, u_{n}\right) \equiv 1+\left[x_{1}, x_{2}, \ldots, x_{n}\right] \quad\left(\bmod F G \Delta(P)^{n+1}\right) .
$$

Como $u_{1}^{-1} u_{2}^{-1} \equiv 1(\bmod F G \Delta(P))$, o Lema 5.3.1 implica

$$
\begin{aligned}
\left(u_{1}, u_{2}\right) & =1+u_{1}^{-1} u_{2}^{-1}\left[u_{1}, u_{2}\right]=1+\left(u_{1}^{-1} u_{2}^{-1}-1\right)\left[u_{1}, u_{2}\right]+\left[u_{1}, u_{2}\right] \\
& \equiv 1+\left[u_{1}, u_{2}\right] \quad\left(\bmod F G \Delta(P)^{3}\right) .
\end{aligned}
$$

Lembramos que os elementos $\widehat{a}_{i}=1+a_{i}+a_{i}^{2}+a_{i}^{3}$ e $1+a_{i}^{2}$, para cada $a_{i} \in Q_{8} \times E$, são centrais em FG. Logo

$$
\begin{aligned}
{\left[u_{1}, u_{2}\right] } & =\left[1-a_{1}\left(1+a_{1}^{2}\right)+x_{1}, 1-a_{2}\left(1+a_{2}^{2}\right)+x_{2}\right] \\
& =\left[x_{1}, x_{2}\right]+\left[a_{1}\left(1+a_{1}^{2}\right), a_{2}\left(1+a_{2}^{2}\right)\right]-\left[x_{1}, a_{2}\left(1+a_{2}^{2}\right)\right]-\left[a_{1}\left(1+a_{1}^{2}\right), x_{2}\right] \\
& =\left[x_{1}, x_{2}\right]+\left[\widehat{a}_{1}, \widehat{a}_{2}\right]-\left[x_{1}, \widehat{a}_{2}\right]-\left[\widehat{a}_{1}, x_{2}\right] \\
& =\left[x_{1}, x_{2}\right]
\end{aligned}
$$

o que prova a congruência (5.22) no caso $n=2$.

Suponha (5.22) verdadeira para $n-1$, isto é,

$$
\left(u_{1}, u_{2}, \ldots, u_{n-1}\right) \equiv 1+\left[x_{1}, x_{2}, \ldots, x_{n-1}\right] \quad\left(\bmod F G \Delta(P)^{n}\right) .
$$


Então o Lema 5.3.1 e como $\left(u_{1}, u_{2}, \ldots, u_{n-1}\right)^{-1} u_{n}^{-1}-1 \in F G \Delta(P)$ implicam

$$
\begin{aligned}
\left(u_{1}, u_{2}, \ldots, u_{n}\right) \\
\quad=1+\left(\left(u_{1}, u_{2}, \ldots, u_{n-1}\right)^{-1} u_{n}^{-1}-1\right)\left[\left(u_{1}, u_{2}, \ldots, u_{n-1}\right), u_{n}\right]+\left[\left(u_{1}, u_{2}, \ldots, u_{n-1}\right), u_{n}\right] \\
\quad \equiv 1+\left[\left(u_{1}, u_{2}, \ldots, u_{n-1}\right), u_{n}\right] \quad\left(\bmod F G \Delta(P)^{n+1}\right) \\
\quad \equiv 1+\left[\left[x_{1}, x_{2}, \ldots, x_{n-1}\right], 1-a_{n}\left(1+a_{n}^{2}\right)+x_{n}\right] \quad\left(\bmod F G \Delta(P)^{n+1}\right) \\
\quad \equiv 1+\left[x_{1}, x_{2}, \ldots, x_{n}\right]-\left[\left[x_{1}, x_{2}, \ldots, x_{n-1}\right], \widehat{a}_{n}\right] \quad\left(\bmod F G \Delta(P)^{n+1}\right) \\
\quad \equiv 1+\left[x_{1}, x_{2}, \ldots, x_{n}\right] \quad\left(\bmod F G \Delta(P)^{n+1}\right)
\end{aligned}
$$

e a afirmação (5.22) é verdadeira, para todo $n \geq 2$.

Seja $n=\mathrm{t}_{\text {nil }}(P)-1$. Se $\mathrm{t}\left((F G)^{+}\right)=\mathrm{t}_{\text {nil }}(P)$, então existem $x_{1}, \ldots, x_{n} \in \mathcal{S}$ tais que $\left[x_{1}, \ldots, x_{n}\right] \neq 0$. Por conseguinte, da congruência $(5.22)$, segue $\gamma_{n}\left(\mathcal{U}^{+}(F G)\right) \neq 1$. Logo $n \leq \operatorname{cl}\left(\mathcal{U}^{+}(F G)\right)$. Por outro lado, $\operatorname{cl}\left(\mathcal{U}^{+}(F G)\right)<\mathrm{t}^{\mathrm{L}}\left((F G)^{+}\right) \leq \mathrm{t}_{\text {nil }}(P)=n+1$, o que mostra (ii).

Suponha que $P$ é poderoso. Então, do Lema 5.3.6, obtemos

$$
x_{n, n} f_{1, \ldots, n}(1-c) a \equiv 2^{n} f_{1, \ldots, n}(1-c) a \quad\left(\bmod F G \Delta(P)^{n+1}\right) .
$$

Além disso, se $h_{i}-1 \in \Delta(P)^{k_{i}}$, então de (5.5) temos

$$
h_{i}-h_{i}^{-1}=\left(h_{i}-1\right)-\left(h_{i}^{-1}-1\right) \equiv 2\left(h_{i}-1\right) \quad\left(\bmod \Delta(P)^{k_{i}+1}\right),
$$

assim

$$
\begin{aligned}
x_{n, n} f_{1, \ldots, n}(1-c) a & \equiv 2^{n}\left(h_{1}-h_{1}^{-1}\right) \cdots\left(h_{n}-h_{n}^{-1}\right)(1-c) a \\
& \equiv 2^{2 n}\left(h_{1}-1\right) \cdots\left(h_{n}-1\right)(1-c) a \quad\left(\bmod F G \Delta(P)^{n+1}\right) .
\end{aligned}
$$

É claro que, se $n<\mathrm{t}_{\text {nil }}(P)$, existem $h_{1}, \ldots, h_{n} \in P$ tais que $\prod_{i=1}^{n}\left(h_{i}-1\right) \neq 0$, e então $x_{n, n} M_{n} \neq 0$. Logo, $\mathrm{t}_{\text {nil }}(P) \leq \mathrm{t}\left((F G)^{+}\right)$e (iii) segue. 
Finalmente, assuma $P$ abeliano. Sejam $a, b \in\left(Q_{8} \times E\right) \backslash \zeta\left(Q_{8} \times E\right)$, e $h_{1}, h_{2} \in P$. Logo

$$
\begin{aligned}
{\left[a\left(h_{1}+a^{2} h_{1}^{-1}\right), b g\left(h_{2}-h_{2}^{-1}\right)\right] } & =\left(h_{2}-h_{2}^{-1}\right)\left(h_{1}+a^{2} h_{1}^{-1}\right)[a, b g] \\
& =g\left(h_{2}-h_{2}^{-1}\right)\left(h_{1}+c h_{1}^{-1}\right)(1-c) a b \\
& =g\left(h_{2}-h_{2}^{-1}\right)\left(h_{1}-h_{1}^{-1}\right)(1-c) a b .
\end{aligned}
$$

e

$$
\begin{aligned}
{\left[a g\left(h_{1}-h_{1}^{-1}\right), b g\left(h_{2}-h_{2}^{-1}\right)\right] } & =\left(h_{2}-h_{2}^{-1}\right)\left(h_{1}-h_{1}^{-1}\right)[a g, b g] \\
& =\left(h_{2}-h_{2}^{-1}\right)\left(h_{1}-h_{1}^{-1}\right) g^{2}(1-c) a b \\
& =-\left(h_{2}-h_{2}^{-1}\right)\left(h_{1}-h_{1}^{-1}\right)(1-c) a b .
\end{aligned}
$$

Então as equações (5.18), (5.24) e (5.25) implicam $\gamma^{2}\left((F G)^{+}\right)=\mathcal{M}_{2}$. Assuma $\gamma^{n-1}\left((F G)^{+}\right)=\mathcal{M}_{n-1}$, para algum $n \geq 3$. Sejam $\alpha \in F P, h \in P$ e $a, b \in\left(Q_{8} \times E\right) \backslash \zeta\left(Q_{8} \times E\right)$. Temos as seguintes igualdades:

$$
\begin{aligned}
{\left[\alpha(1-c) a, g b\left(h-h^{-1}\right)\right] } & =\alpha\left(h-h^{-1}\right)(1-c)[a, g b] \\
& =g \alpha\left(h-h^{-1}\right)(1-c)[a, b] \\
& =g \alpha\left(h-h^{-1}\right)(1-c)^{2} a b \\
& =2 g \alpha\left(h-h^{-1}\right)(1-c) a b,
\end{aligned}
$$

e

$$
\begin{aligned}
{\left[g \alpha(1-c) a, g b\left(h-h^{-1}\right)\right] } & =g^{2} \alpha\left(h-h^{-1}\right)(1-c)[a, b] \\
& =c \alpha\left(h-h^{-1}\right)(1-c)[a, b] \\
& =c \alpha\left(h-h^{-1}\right)(1-c)^{2} a b \\
& =-2 \alpha\left(h-h^{-1}\right)(1-c) a b .
\end{aligned}
$$

Substituindo $f_{1, \ldots, n-1}$ por $\alpha$ nas equações (5.18), (5.26) e (5.27), obtemos $\left[\mathcal{M}_{n-1},(F G)^{+}\right]=\mathcal{M}_{n}$, donde

$$
\gamma^{n}\left((F G)^{+}\right)=\left[\gamma^{n-1}\left((F G)^{+}\right),(F G)^{+}\right]=\left[\mathcal{M}_{n-1},(F G)^{+}\right]=\mathcal{M}_{n}
$$


como queríamos provar. 


\section{Capítulo 6}

\section{Conclusões}

\subsection{Considerações finais}

O objetivo desta tese foi estudar as propriedades de Lie, definidas no Capítulo 1, dos elementos simétricos sob uma involução clássica orientada. Em alguns casos, também foram obtidos resultados para o conjunto dos elementos anti-simétricos.

Os principais resultados da tese, foram os seguintes. Na Seção 3.1, provamos que se $G$ é um grupo tal que $\zeta(G)^{2}=\left\{z^{2}: z \in \zeta(G)\right\}$ é um conjunto infinito, os índices de Lie nilpotência de $(F G)^{+}$e $F G$ são iguais. Na Seção 3.2 para grupos sem elementos de ordem 2, provamos que se $(F G)^{+}$é Lie $n$-Engel, para algum $n \in \mathbb{N}$ ou Lie nilpotente, então a álgebra de grupo $F G$ também satisfaz a respectiva propriedade de Lie.

Para álgebras de grupo $F G$, para grupos $G$ que contêm uma cópia do grupo quatérnio de ordem 8, na Seção 4.1 damos uma caracterização completa de quando o conjunto dos elementos simétricos sob uma involução clássica orientada satisfaz uma das três propriedades de Lie estudadas nesta tese. Para esta classe de grupos mostramos que seu conjunto de unidades simétricas é nilpotente. No caso em que o grupo não contém uma cópia de $Q_{8}$ foram dados alguns resultados parciais, para grupos nos quais se $(F G)^{+}$satisfaz uma das propriedades de Lie, então a álgebra de grupo $F G$ também o faz. Aqui ainda foi apresentado um exemplo que justifica porque os resultados que foram obtidos em pesquisas anteriores não podem ser obtidos neste contexto.

Finalmente, no Capítulo 5, foi estudado quando o índice de Lie nilpotência de $(F G)^{+}$e 
a classe de nilpotência de $\mathcal{U}^{+}(F G)$ atingem o maior limitante possível em álgebras de grupo Lie nilpotentes. Além disso, foram dados alguns resultados sobre o índice de nilpotência de $(F G)^{+}$e a classe de nilpotência de $\mathcal{U}^{+}(F G)$, em grupos tais que $(F G)^{+}$é Lie nilpotente e $G$ contém uma cópia de $Q_{8}$.

\subsection{Sugestões para pesquisas futuras}

Como perspectiva para dar continuidade à pesquisa feita nesta tese, podem ser considerados os seguintes tópicos:

- Dar uma caracterização completa para grupos que não contêm $Q_{8}$, para as propriedades de Lie estudadas.

- Estudar outras propriedades de Lie em elementos simétricos sob uma involução clássica orientada.

- Estudar propriedades de Lie de elementos simétricos sob involuções de grupo orientadas, isto é so involuções da álgebra de grupo $F G$ definidas por

$$
\left(\sum_{g \in G} \alpha_{g} g\right)^{\sharp}=\sum_{g \in G} \alpha_{g} \sigma(g) g^{*},
$$

onde * é uma involução de $G$ e $\sigma$ é uma orientação de $G$.

- Estudar quando o conjunto das unidades simétricas é nilpotente, $n$-Engel, solúvel, etc.

- Estudar índices de nilpotência do conjunto dos elementos simétricos sob outras involuções. 


\section{Referências Bibliográficas}

[1] S. A. Amitsur. Rings with involution. Israel J. Math., 6:99-106, 1968. 1

[2] C. Bagiński. A note on the derived length of the unit group of a modular group algebra. Comm. Algebra, 30(10):4905-4913, 2002. 41

[3] Z. Balogh e T. Juhász. Derived lengths of symmetric and skew symmetric elements in group algebras. JP J. Algebra Number Theory Appl., 12(2):191-203, 2008. 49

[4] Z. Balogh e T. Juhász. Nilpotency class of symmetric units of group algebras. Publ. Math. Debrecen, 79(1-2):171-180, 2011. 5, 39

[5] Z. Balogh e T. Juhász. Nilpotency indices of symmetric elements of group algebras. Comm. Algebra, 40(11):4283-4294, 2012. 5, 6, 39, 45, 48, 56, 57

[6] V. Bovdi e E. Spinelli. Modular group algebras with maximal Lie nilpotency indices. Publ. Math. Debrecen, 65(1-2):243-252, 2004. 5, 39, 49

[7] O. Broche Cristo. Commutativity of symmetric elements in group rings. J. Group Theory, 9(5):673-683, 2006. 5

[8] O. Broche Cristo, E. Jespers, C. Polcino Milies, e M. Ruiz Marín. Antisymmetric elements in group rings. II. J. Algebra Appl., 8(1):115-127, 2009. 5

[9] O. Broche Cristo, E. Jespers, e M. Ruiz Marín. Antisymmetric elements in group rings with an orientation morphism. Forum Math., 21(3):427-454, 2009. 5

[10] O. Broche Cristo e C. Polcino Milies. Symmetric elements under oriented involutions in group rings. Comm. Algebra, 34(9):3347-3356, 2006. 5, 10

[11] J. H. Castillo e C. Polcino Milies. Lie properties of symmetric elements under oriented involutions. Comm. Algebra, to appear 2012. 6

[12] A. Giambruno e C. Polcino Milies. Unitary units and skew elements in group algebras. Manuscripta Math., 111(2):195-209, 2003. 5

[13] A. Giambruno, C. Polcino Milies, e S. K. Sehgal. Lie properties of symmetric elements in group rings. J. Algebra, 321(3):890-902, 2009. 4, 10

[14] A. Giambruno e S. K. Sehgal. A Lie property in group rings. Proc. Amer. Math. Soc., 105(2):287-292, 1989. 25

[15] A. Giambruno e S. K. Sehgal. Lie nilpotence of group rings. Comm. Algebra, 21(11):4253-4261, 1993. 4, 5, 6 
[16] A. Giambruno e S. K. Sehgal. Group algebras whose Lie algebra of skew-symmetric elements is nilpotent. In Groups, rings and algebras, volume 420 of Contemp. Math., pages 113-120. Amer. Math. Soc., Providence, RI, 2006. 5

[17] I. N. Herstein. Rings with involution. The University of Chicago Press, Chicago, Ill.London, 1976. Chicago Lectures in Mathematics. 27

[18] G. Higman. The units of group-rings. Proc. London Math. Soc. (2), 46:231-248, 1940. 2

[19] I. M. Isaacs e D. S. Passman. Groups with representations of bounded degree. Canad. J. Math., 16:299-309, 1964. 7

[20] S. A. Jennings. The structure of the group ring of a $p$-group over a modular field. Trans. Amer. Math. Soc., 50:175-185, 1941. 16, 56

[21] E. Jespers e M. Ruiz Marín. On symmetric elements and symmetric units in group rings. Comm. Algebra, 34(2):727-736, 2006. 2, 5

[22] G. T. Lee. Group rings whose symmetric elements are Lie nilpotent. Proc. Amer. Math. Soc., 127(11):3153-3159, 1999. 4, 6, 9, 29, 37

[23] G. T. Lee. The Lie $n$-Engel property in group rings. Comm. Algebra, 28(2):867-881, 2000. 4, 6, 9, 29

[24] G. T. Lee. Group identities on units and symmetric units of group rings, volume 12 of Algebra and Applications. Springer-Verlag London Ltd., London, 2010. 3, 6, 9, 28, 33, $35,36,38$

[25] G. T. Lee, S. K. Sehgal, e E. Spinelli. Lie properties of symmetric elements in group rings. II. J. Pure Appl. Algebra, 213(6):1173-1178, 2009. 5

[26] S. P. Novikov. Algebraic construction and properties of Hermitian analogs of $K$-theory over rings with involution from the viewpoint of Hamiltonian formalism. Applications to differential topology and the theory of characteristic classes. I. II. Izv. Akad. Nauk SSSR Ser. Mat., 34:253-288; ibid. 34 (1970), 475-500, 1970. 5

[27] I. B. S. Passi, D. S. Passman, e S. K. Sehgal. Lie solvable group rings. Canad. J. Math., 25:748-757, 1973. 4, 8

[28] D. S. Passman. Group rings satisfying a polynomial identity. J. Algebra, 20:103-117, 1972. 7

[29] D. S. Passman. The algebraic structure of group rings. Pure and Applied Mathematics. Wiley-Interscience [John Wiley \& Sons], New York, 1977. 7, 10, 13, 15, 28

[30] C. Polcino Milies e S. K. Sehgal. An introduction to group rings, volume 1 of Algebras and Applications. Kluwer Academic Publishers, Dordrecht, 2002. 12, 13, 25, 26, 55, 57

[31] D. J. S. Robinson. A course in the theory of groups, volume 80 of Graduate Texts in Mathematics. Springer-Verlag, New York, second edition, 1996. 38

[32] S. K. Sehgal. Topics in group rings, volume 50 of Monographs and Textbooks in Pure and Applied Math. Marcel Dekker Inc., New York, 1978. 4, 8 
[33] R. K. Sharma e V. Bist. A note on Lie nilpotent group rings. Bull. Austral. Math. Soc., 45(3):503-506, 1992. 5, 39 


\section{Índice Remissivo}

p-elemento, 17

$p^{\prime}$-elemento, 17

altura, 14

Amitsur, S. A., 1

anel de grupo, 1

aumento

aplicação de, 12

ideal de, 12

Balogh, Zsolt, 5

Bist, Vikas, 5

Bovdi, V., 5

Broche Cristo, 5

classe de nilpotência, 4

conjunto

elementos anti-simétricos, 1

elementos simétricos, 1

nilpotente, 3

unidades simétricas, 4

elemento

anti-simétrico, 1

simétrico, 1

FC subgrupo, 7

filtração, 13

Giambruno, A., 4

grupo

$p$-abeliano, 7

diedral de ordem $2 k, 23$

diedral infinito, 23

poderoso, 56

simétrico de grau $r, 9$

unidades, 4

Higman, G., 2

identidade polinomial

standard de grau $r, 9$

indice
Lie nilpotência, 3

Lie nilpotência superior, 3

nilpotência, 57

involução, 1

clássica, 2

Jespers, E., 2

Juhász, Tibor, 5

Lee, G.T, 4

Lie

$n$-Engel, 3

colchete de, 2

nilpotente

fortemente, 3

nilpotente, 3

série central inferior, 3

série de potências, 3

Passi, I.B.S., 4

Passman, D.S., 4

peso, 15

PI-Álgebra, 7

Polcino Milies, C., 4

produto central interno, 29

Ruiz Marín, 2

série central inferior, 3

Sehgal, S.K., 4

sequência

$$
\begin{aligned}
& N-, 14 \\
& N_{p}-, 14
\end{aligned}
$$

Sharma, R.K, 5

Spinelli, E., 4, 5

subgrupo comutador, 7

subgrupos dimensionais, 14

suporte, 1 